Supporting Information for:

\title{
Multiple Bonding in Lanthanides and Actinides: Direct Comparison of Covalency in Thorium(IV)- and Cerium(IV)-Imido Complexes
}

Thibault Cheisson, ${ }^{\dagger, \S}$ Kyle D. Kersey, ${ }^{\dagger} \S$ Nolwenn Mahieu, ${ }^{\dagger, \star, \S}$ Alex McSkimming, ${ }^{\dagger}$ Michael R. Gau, ${ }^{\dagger}$ Patrick J. Carroll, ${ }^{\dagger}$ and Eric J. Schelter ${ }^{*}{ }^{\dagger}$

${ }^{\dagger}$ P. Roy and Diana T. Vagelos Laboratories, Department of Chemistry, University of Pennsylvania, 231 S 34th St., Philadelphia, Pennsylvania 19104, United States

${ }^{\sharp}$ ENS Paris-Saclay, Département de Chimie, Université Paris-Saclay, 94235 Cachan, France

${ }^{\S}$ These authors contributed equally

*Email: schelter@sas.upenn.edu

\section{Table of contents}

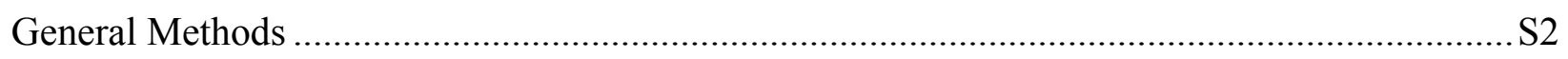

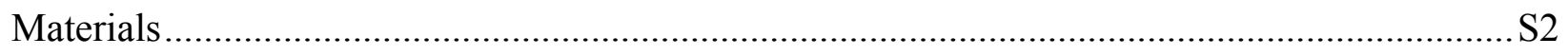

Synthetic Procedures and Spectroscopic Data ………………................................................... 3

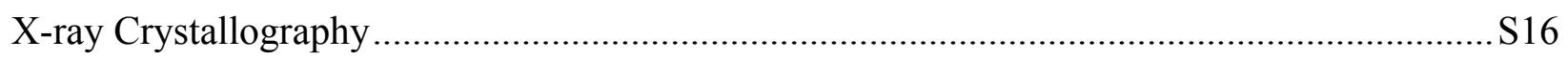

Electronic Absorption Spectroscopy …………………........................................................ 22

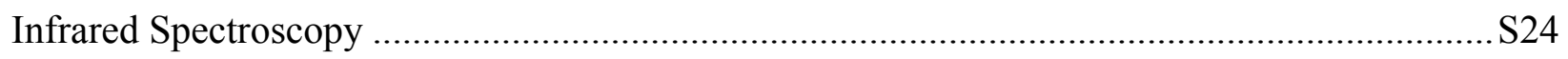

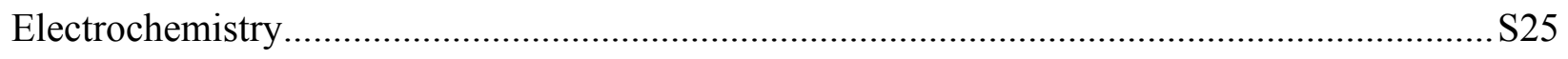

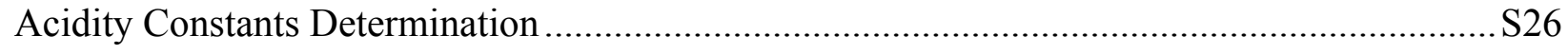

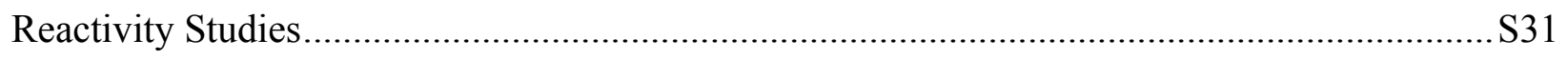

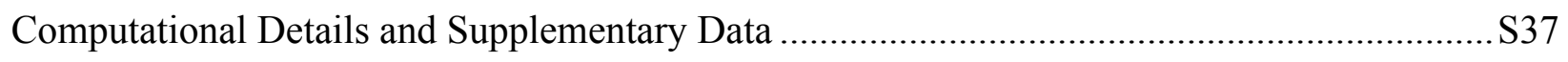

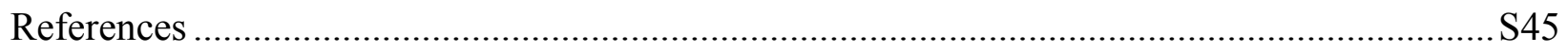


General Methods. All synthesis and handling were performed under an inert $\mathrm{N}_{2}$ atmosphere in a drybox equipped with a molecular sieves 13X/Q5 $\mathrm{Cu}-0226 \mathrm{~S}$ catalyst purifier system. All glassware used was oven-dried at $150{ }^{\circ} \mathrm{C}$ overnight prior to use. Nuclear Magnetic Resonance (NMR) analyses $\left({ }^{1} \mathrm{H},{ }^{2} \mathrm{H},{ }^{13} \mathrm{C}\left\{{ }^{1} \mathrm{H}\right\},{ }^{7} \mathrm{Li},{ }^{11} \mathrm{~B}\left\{{ }^{1} \mathrm{H}\right\}\right.$, and $\left.{ }^{19} \mathrm{~F}\left\{{ }^{1} \mathrm{H}\right\}\right)$ were performed on Bruker AVIII400, DRX-500, and AVIII-500 Fourier Transform NMR spectrometers. The spectra were recorded at $300 \mathrm{~K}$. Chemical shifts are reported in units of parts per million (ppm), and referenced against proteo-solvent residual peaks $\left({ }^{1} \mathrm{H}\right)$ and characteristic solvent peaks $\left({ }^{13} \mathrm{C}\right)$ or appropriate external references $\left({ }^{7} \mathrm{Li},{ }^{11} \mathrm{~B},{ }^{19} \mathrm{~F}\right)$. The following abbreviations were used: $\mathrm{d}$, doublet; t, triplet; q, quartet; sept, septet; m, multiplet. Elemental analyses were performed on a Costech ECS 4010 analyzer.

Caution! Natural thorium (primary isotope ${ }^{232} \mathrm{Th}$ ) is a weak $\alpha$-emitter $(4.012 \mathrm{MeV}$ ) with a halflife of $1.41 \times 10^{10}$ years; manipulations and reactions should be carried out in monitored fume hoods or in an inert atmosphere drybox in a radiation laboratory equipped with counting equipment.

Materials. Diethyl ether $\left(\mathrm{Et}_{2} \mathrm{O}\right)$, dichloromethane (DCM), tetrahydrofuran (THF), n-pentane, and toluene were dried using a commercial two-column Q5 reactant- and neutral alumina-packed system (one column of each). $\mathrm{H}_{3}$ TriNOx, ${ }^{1} \mathrm{ThCl}_{4}(\mathrm{DME})_{2},{ }^{2} \mathrm{KBn}^{3}{ }^{3} \mathrm{NaBAr}_{4}{ }_{4}\left(\mathrm{Ar}^{\mathrm{F}}=3,5-\right.$ bis(trifluoromethyl)phenyl), ${ }^{4}$ and $\left[{ }^{n} \operatorname{Pr}_{4} \mathrm{~N}\right]\left[\mathrm{BAr}_{4}{ }_{4}\right]^{5}$ were synthesized based on reported procedures. All deuterated solvents were obtained from Cambridge Isotope Laboratories. $\mathrm{C}_{6} \mathrm{D}_{6}$ was dried over a sodium mirror, $\mathrm{CD}_{2} \mathrm{Cl}_{2}$ was dried over $4 \AA$ molecular sieves, THF- $d_{8}$ and toluene- $d_{8}$ were dried over $\mathrm{Na} /$ benzophenone and distilled prior to use. $\mathrm{LiN}\left(\mathrm{SiMe}_{3}\right)_{2}(\mathrm{Sigma}$ Aldrich) was used as received. 3,5-bis(trifluoromethyl)aniline $\left(\mathrm{Ar}^{\mathrm{F}} \mathrm{NH}_{2}\right.$, Oakwood) was dried over $4 \AA$ molecular sieves prior to use. $\mathrm{LiCH}_{2} \mathrm{SiMe}_{3}$ (Sigma Aldrich) was obtained as an $n$ pentane solution, dried, and recrystallized from $n$-pentane at $-20{ }^{\circ}$ C. 2.2.2-cryptand (Sigma Aldrich) was dissolved in $\mathrm{Et}_{2} \mathrm{O}$ and dried over $4 \AA$ molecular sieves for 3 days prior to use. 


\section{Synthetic Procedures and Characterization Data.}

[ThCl(TriNOx)] (1): To a clear, colorless solution of $\mathrm{ThCl}_{4}(\mathrm{DME})_{2}$ (2.24 g, $4.04 \mathrm{mmol}, 1$ equiv) in THF $(20 \mathrm{~mL})$ was added a solution of $\mathrm{H}_{3}$ TriNOx (2.00 g, $3.64 \mathrm{mmol}, 0.9$ equiv) in THF $(20 \mathrm{~mL})$. With stirring, a solution of $\mathrm{LiN}\left(\mathrm{SiMe}_{3}\right)_{2}(2.03 \mathrm{~g}, 12.16 \mathrm{mmol}, 3$ equiv) in THF $(15 \mathrm{~mL})$ was added dropwise, causing the immediate precipitation of a white solid. After stirring overnight, the solid precipitate was isolated on a fine-porosity fritted filter and washed with THF $(3 \mathrm{~mL})$ then with $\mathrm{Et}_{2} \mathrm{O}(20 \mathrm{~mL})$. The product was dried

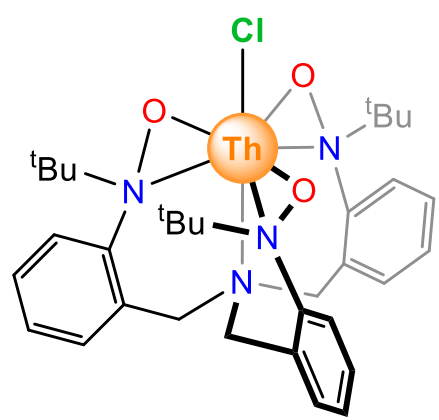
under reduced pressure and collected as a white powder. Yield: $2.29 \mathrm{~g}, 70 \%$.

${ }^{1} \mathbf{H}$ NMR $\left(360 \mathrm{MHz}, \mathrm{CD}_{2} \mathrm{Cl}_{2}\right): \delta 7.66\left(\mathrm{~d},{ }^{3} \mathrm{~J}_{\mathrm{HH}}=8.0 \mathrm{~Hz}, 3 \mathrm{H}, \mathrm{Ar}-\mathrm{H}\right), 7.41-7.44(\mathrm{~m}, 3 \mathrm{H}, \mathrm{Ar}-\mathrm{H})$, 7.26-7.32 (m, 6H, Ar-H), $4.49\left(\mathrm{~d},{ }^{2} J_{\mathrm{HH}}=12.5 \mathrm{~Hz}, 3 \mathrm{H}, \mathrm{CH}_{2}\right), 2.95\left(\mathrm{~d},{ }^{2} J_{\mathrm{HH}}=12.5 \mathrm{~Hz}, 3 \mathrm{H}, \mathrm{CH}_{2}\right)$, $0.81\left(\mathrm{~s}, 27 \mathrm{H}, \mathrm{C}\left(\mathrm{CH}_{3}\right)_{3}\right)$.

${ }^{13} \mathbf{C}\left\{{ }^{1} \mathrm{H}\right\}$ NMR $\left(125.66 \mathrm{MHz}, \mathrm{CD}_{2} \mathrm{Cl}_{2}\right): \delta 147.8,132.5,131.4,130.4,130.1,127.6,63.5\left(\mathrm{CH}_{2}\right)$, $61.8\left(\mathrm{C}\left(\mathrm{CH}_{3}\right)_{3}\right), 26.0\left(\mathrm{C}\left(\mathrm{CH}_{3}\right)_{3}\right)$.

Anal. Calc. for $\mathbf{C}_{33} \mathbf{H}_{45} \mathbf{C l N}_{4} \mathbf{O}_{3}$ Th: C, 48.74; H, 5.58; N, 6.89. Found: C, 49.01; H, 5.70; N, 6.52.

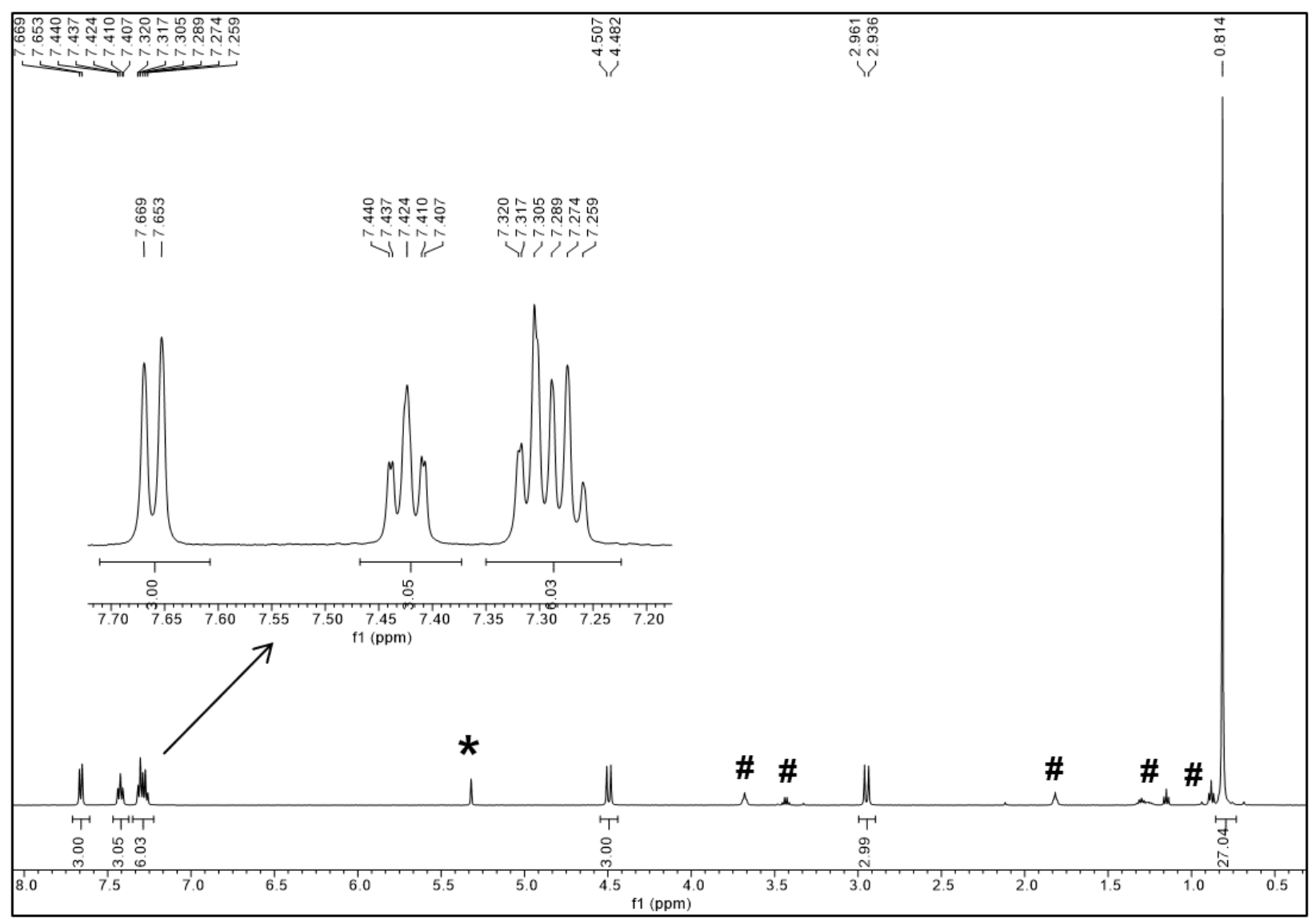

Figure S1. ${ }^{1} \mathrm{H}$ NMR spectrum of $[\mathrm{ThCl}(\operatorname{TriNOx})]$ (1) in $\mathrm{CD}_{2} \mathrm{Cl}_{2}\left({ }^{*}\right)$, \# corresponds to trace solvent signals. 


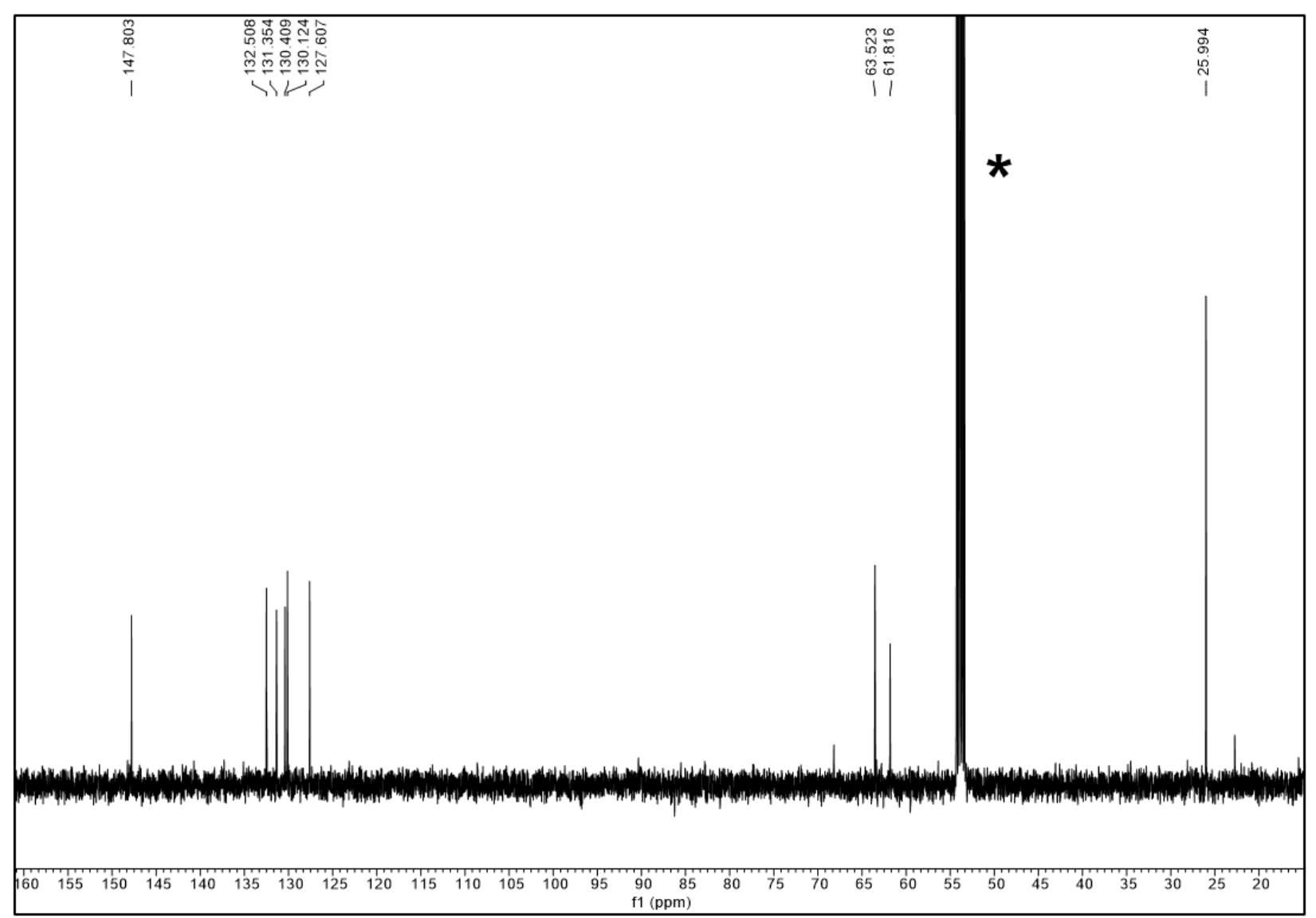

Figure S2. ${ }^{13} \mathrm{C}\left\{{ }^{1} \mathrm{H}\right\}$ NMR spectrum for $[\mathrm{ThCl}(\operatorname{TriNOx})](\mathbf{1})$ in $\mathrm{CD}_{2} \mathrm{Cl}_{2}\left({ }^{*}\right)$.

[Th( $\left.\mathrm{CH}_{2} \mathrm{SiMe}_{3}\right)$ (TriNOx)] (2): [ThCl(TriNOx)] (1) (470 mg, 0.58 mmol, 1.0 equiv) was suspended in THF $(5 \mathrm{~mL})$. With stirring, a THF solution $(2 \mathrm{~mL})$ of $\mathrm{LiCH}_{2} \mathrm{SiMe}_{3}(80.9 \mathrm{mg}, 0.86 \mathrm{mmol}, 1.5$ equiv) was added dropwise, causing rapid clarification of the suspension and the appearance of a pale-yellow color. After stirring for 15 minutes, the solvent was removed under reduced pressure, and the solid was washed sequentially with $n$-pentane $(2 \times 2 \mathrm{~mL})$ and $\mathrm{Et}_{2} \mathrm{O}(2 \times 2 \mathrm{~mL})$ before decanting the supernatant and drying the

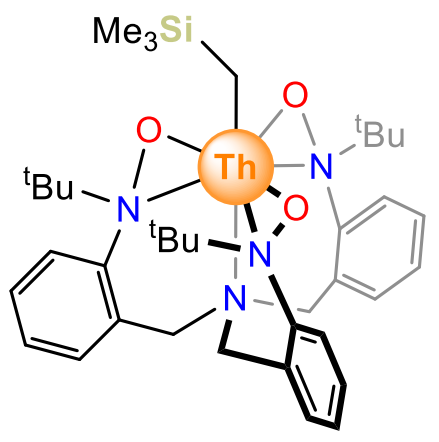
solid under reduced pressure to produce a fine white powder. Yield: $398 \mathrm{mg}, 80 \%$.

${ }^{1} \mathbf{H}$ NMR $\left(360 \mathrm{MHz}, \mathrm{C}_{6} \mathrm{D}_{6}\right): \delta 7.83\left(\mathrm{dd},{ }^{3} J_{\mathrm{HH}}=8.0 \mathrm{~Hz},{ }^{4} J_{\mathrm{HH}}=1.0 \mathrm{~Hz}, 3 \mathrm{H}, \mathrm{Ar}-\mathrm{H}\right), 7.09(\mathrm{~m}, 3 \mathrm{H}$, Ar-H), $6.92\left(\mathrm{td},{ }^{3} J_{\mathrm{HH}}=7.5 \mathrm{~Hz},{ }^{4} J_{\mathrm{HH}}=1.0 \mathrm{~Hz}, 3 \mathrm{H}, \mathrm{Ar}-\mathrm{H}\right), 6.87\left(\mathrm{dd},{ }^{3} J_{\mathrm{HH}}=8.0 \mathrm{~Hz} \mathrm{~Hz},{ }^{4} J_{\mathrm{HH}}=1.5\right.$ $\mathrm{Hz}, 3 \mathrm{H}, \mathrm{Ar}-\mathrm{H}), 4.31\left(\mathrm{~d},{ }^{2} J_{\mathrm{HH}}=11.5 \mathrm{~Hz}, 3 \mathrm{H}, \mathrm{CH}_{2}\right), 2.29\left(\mathrm{~d},{ }^{2} J_{\mathrm{HH}}=12.0 \mathrm{~Hz}, 3 \mathrm{H}, \mathrm{CH}_{2}\right), 0.90(\mathrm{~s}$, $\left.27 \mathrm{H}, \mathrm{C}\left(\mathrm{CH}_{3}\right)_{3}\right), 0.73\left(\mathrm{~s}, 9 \mathrm{H}, \mathrm{Si}\left(\mathrm{CH}_{3}\right)_{3}\right), 0.66\left(\mathrm{~d},{ }^{2} \mathrm{~J}_{\mathrm{HH}}=2.0 \mathrm{~Hz}, 2 \mathrm{H}, \mathrm{Th}-\mathrm{CH}_{2}\right)$.

${ }^{13} \mathbf{C}\left\{{ }^{1} \mathbf{H}\right\}$ NMR (125.66 MHz, $\left.\mathrm{C}_{6} \mathrm{D}_{6}\right): \delta$ 149.9, 132.0, 131.9, 130.8, 129.3, $62.9\left(\mathrm{CH}_{2}\right.$, TriNOx), $61.2\left(\mathrm{C}_{\left(\mathrm{CH}_{3}\right)_{3}}\right.$, TriNOx), $59.7\left(\mathrm{Th}-\mathrm{CH}_{2}\right), 26.0\left(\mathrm{C}\left(\mathrm{CH}_{3}\right)_{3}\right.$, TriNOx $), 5.2\left(\mathrm{Si}\left(\mathrm{CH}_{3}\right)_{3}\right)$.

Anal. Calc. for $\mathbf{C}_{37} \mathbf{H}_{56} \mathbf{N}_{4} \mathbf{O}_{3}$ SiTh: C, 51.38; H, 6.53; N, 6.48. Found: C, 51.48; H, 6.21; N, 6.24. 


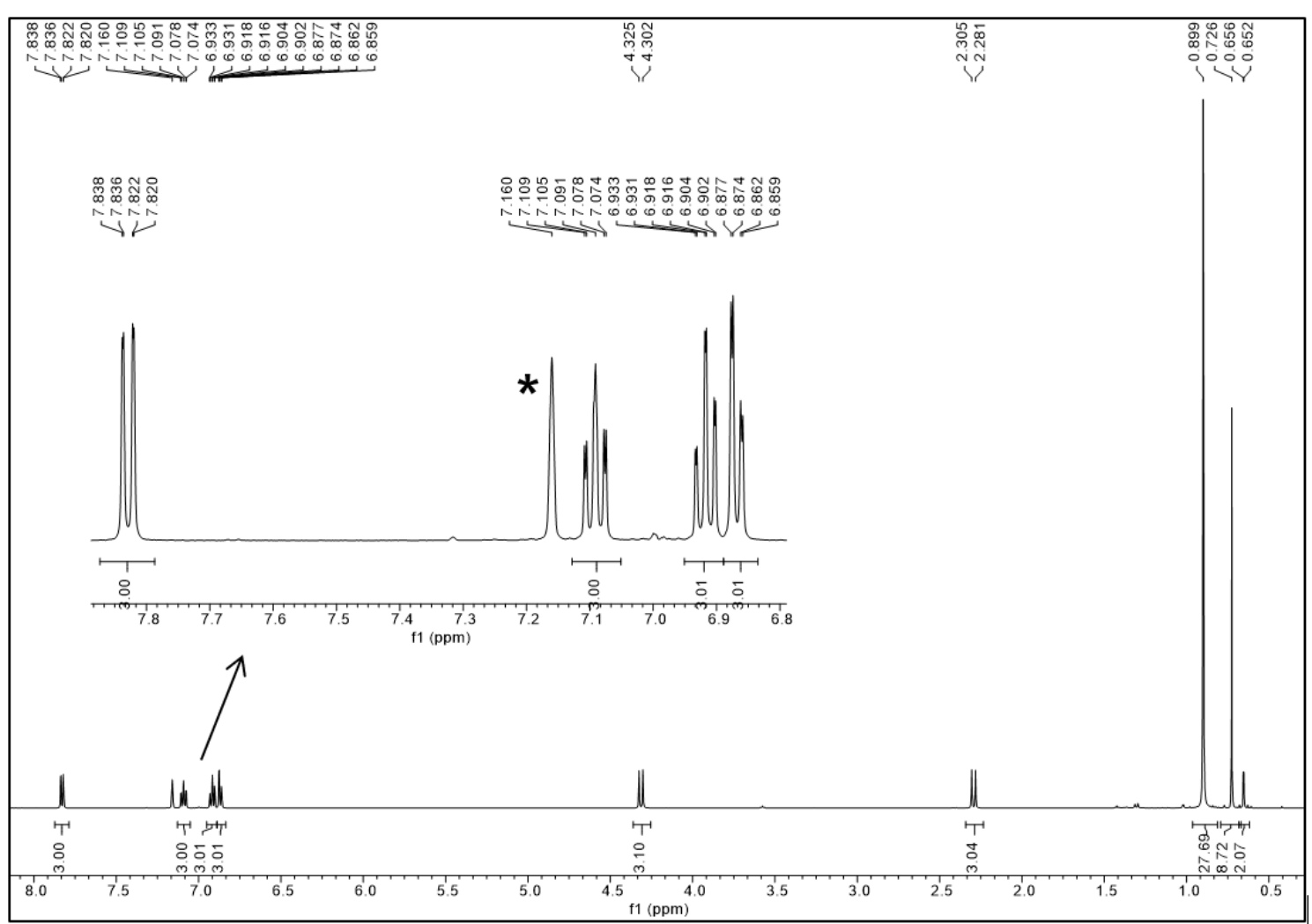

Figure S3. ${ }^{1} \mathrm{H}$ NMR spectrum of $\left[\mathrm{Th}\left(\mathrm{CH}_{2} \mathrm{SiMe}_{3}\right)(\mathrm{TriNOx})\right](2)$ in $\mathrm{C}_{6} \mathrm{D}_{6}(*)$.

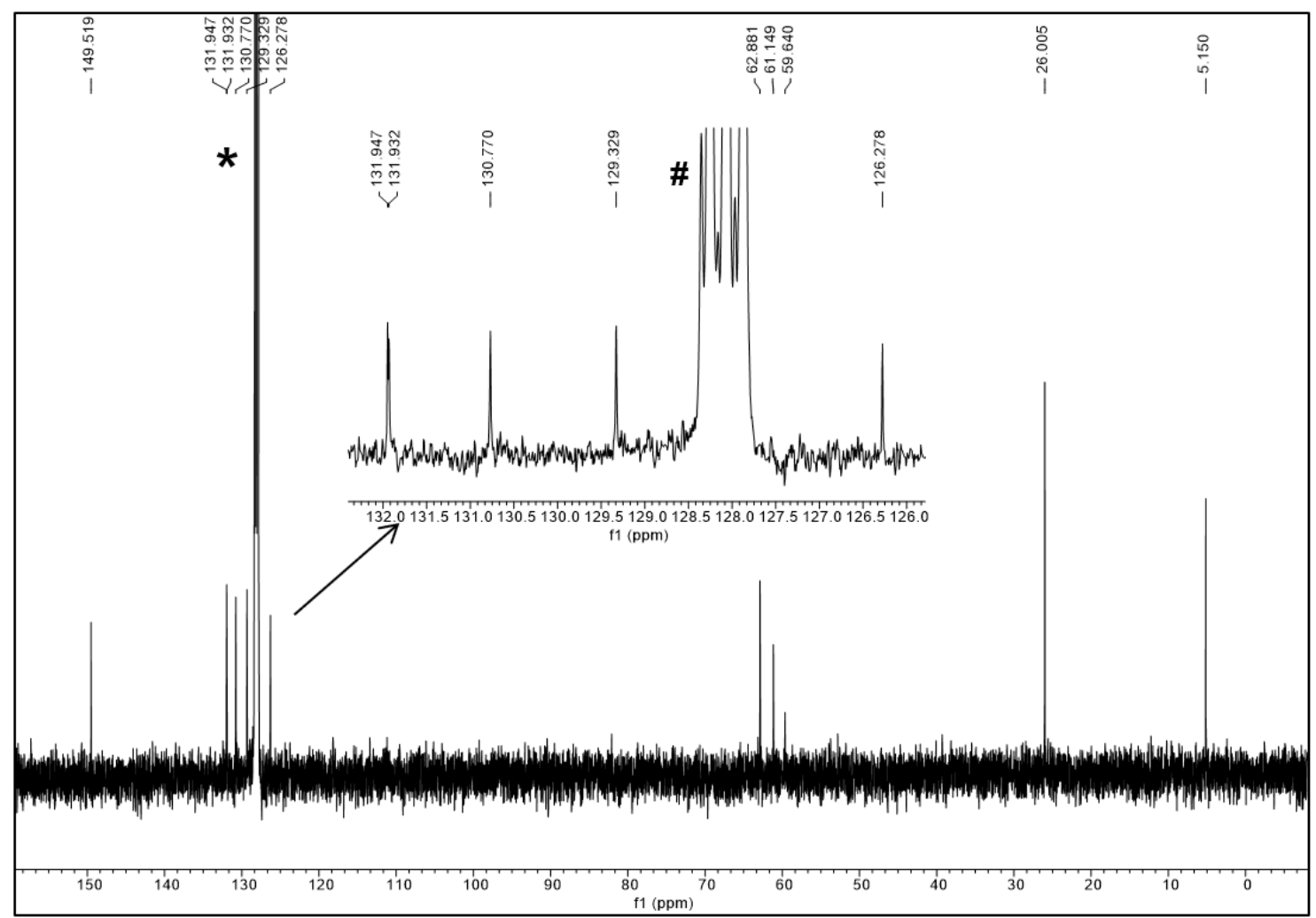

Figure S4. ${ }^{13} \mathrm{C}\left\{{ }^{1} \mathrm{H}\right\}$ NMR spectrum of $\left[\mathrm{Th}\left(\mathrm{CH}_{2} \mathrm{SiMe}_{3}\right)(\operatorname{TriNOx})\right](2)$ in $\mathrm{C}_{6} \mathrm{D}_{6}\left({ }^{*}\right)$. 
[Th(NHAr $\left.{ }^{\mathbf{F}}\right)$ (TriNOx)] (3): [Th( $\left.\mathrm{CH}_{2} \mathrm{SiMe}_{3}\right)($ TriNOx) $]$ (2) (75 mg, $87 \mu \mathrm{mol}, 1.0$ equiv) was suspended in $\mathrm{Et}_{2} \mathrm{O}(3 \mathrm{~mL})$. With stirring, an $\mathrm{Et}_{2} \mathrm{O}$ solution of $\mathrm{Ar}^{\mathrm{F}} \mathrm{NH}_{2}$ (45 mg, $0.20 \mathrm{mmol}, 2.2$ equiv) was added dropwise, initially forming a yellow color. The reaction mixture was stirred for 1.5 hours, during which time the solution further clouded, forming a milky-white precipitate. The solid was collected by filtration over a fine-porosity fritted filter and sequentially washed with $\mathrm{Et}_{2} \mathrm{O}(2 \times 2 \mathrm{~mL})$ and $n$-pentane $(2 \times 2 \mathrm{~mL})$ before being dried under reduced pressure and collected to produce a white powder. Yield: $77 \mathrm{mg}, 88 \%$.

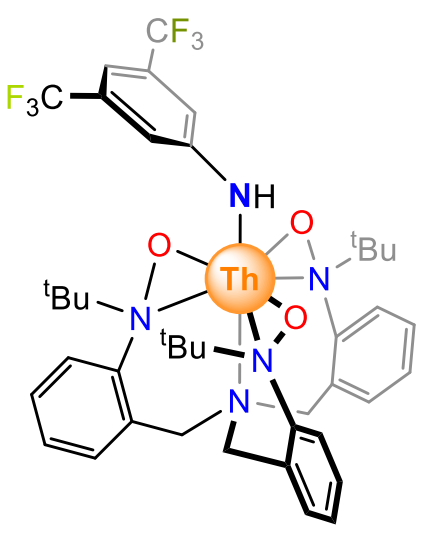

${ }^{1} \mathbf{H}$ NMR $\left(500 \mathrm{MHz}, \mathrm{CD}_{2} \mathrm{Cl}_{2}\right): \delta 7.68\left(\mathrm{dd},{ }^{3} J_{\mathrm{HH}}=8.2 \mathrm{~Hz},{ }^{4} J_{\mathrm{HH}}=1.3 \mathrm{~Hz}, 3 \mathrm{H}, \mathrm{Ar}-\mathrm{H}\right), 7.43$ (ddd, $\left.{ }^{3} J_{\mathrm{HH}}=8.3 \mathrm{~Hz},{ }^{3} J_{\mathrm{HH}}=7.0 \mathrm{~Hz},{ }^{4} J_{\mathrm{HH}}=1.9 \mathrm{~Hz}, 3 \mathrm{H}, \mathrm{Ar}-\mathrm{H}\right), 7.25-7.34(\mathrm{~m}, 6 \mathrm{H}, \mathrm{Ar}-\mathrm{H}), 7.31(\mathrm{~s}, 2 \mathrm{H}, o-$ $\left.\mathrm{H}, \mathrm{Ar}^{\mathrm{F}}\right), 6.66\left(\mathrm{~s}, 1 \mathrm{H}, p-\mathrm{H}, \mathrm{Ar}^{\mathrm{F}}\right), 5.80(\mathrm{~s}, 1 \mathrm{H}, \mathrm{NH}), 4.54\left(\mathrm{~d},{ }^{2} J_{\mathrm{HH}}=12.1 \mathrm{~Hz}, 3 \mathrm{H}, \mathrm{CH}_{2}\right), 2.94(\mathrm{~d}$, $\left.{ }^{2} J_{\mathrm{HH}}=12.2 \mathrm{~Hz}, 3 \mathrm{H}, \mathrm{CH}_{2}\right), 0.80\left(\mathrm{~s}, 27 \mathrm{H}, \mathrm{C}\left(\mathrm{CH}_{3}\right)_{3}\right)$.

${ }^{13} \mathbf{C}\left\{{ }^{1} \mathbf{H}\right\}$ NMR $\left(125.66 \mathrm{MHz}, \mathrm{CD}_{2} \mathrm{Cl}_{2}\right): \delta 161.0\left(\mathrm{HN}-C, \mathrm{Ar}^{\mathrm{F}}\right), 148.1,132.6,131.5,131.3\left(\mathrm{q},{ }^{2} J_{\mathrm{FC}}\right.$ $\left.=31.2 \mathrm{~Hz}, C-\mathrm{CF}_{3}, \mathrm{Ar}^{\mathrm{F}}\right), 130.4,130.1,127.5,125.2\left(\mathrm{q},{ }^{1} J_{\mathrm{FC}}=272.5 \mathrm{~Hz}, C F_{3}, \mathrm{Ar}^{\mathrm{F}}\right), 116.1\left(\mathrm{q},{ }^{3} J_{\mathrm{FC}}\right.$ $\left.=2.4 \mathrm{~Hz}, o-\mathrm{C}, \mathrm{Ar}^{\mathrm{F}}\right), 103.6$ (sept., $\left.{ }^{3} J_{\mathrm{FC}}=3.8 \mathrm{~Hz}, p-\mathrm{C}, \mathrm{Ar}^{\mathrm{F}}\right), 63.39\left(C\left(\mathrm{CH}_{3}\right)_{3}\right.$, TriNOx), 61.47 $\left(\mathrm{CH}_{2}\right.$, TriNOx), $26.1\left(\mathrm{C}\left(\mathrm{CH}_{3}\right)_{3}\right.$, TriNOx).

${ }^{19} \mathbf{F}\left\{{ }^{1} \mathbf{H}\right\}$ NMR $\left(376.30 \mathrm{MHz}, \mathrm{CD}_{2} \mathrm{Cl}_{2}\right): \delta-63.24(\mathrm{~s})$

Anal. Calc. for $\mathbf{C}_{41} \mathbf{H}_{49} \mathbf{F}_{6} \mathbf{N}_{5} \mathbf{O}_{3}$ Th: C, 48.96; H, 4.91; N, 6.96. Found: C, 49.16; H, 5.01; N, 6.73.

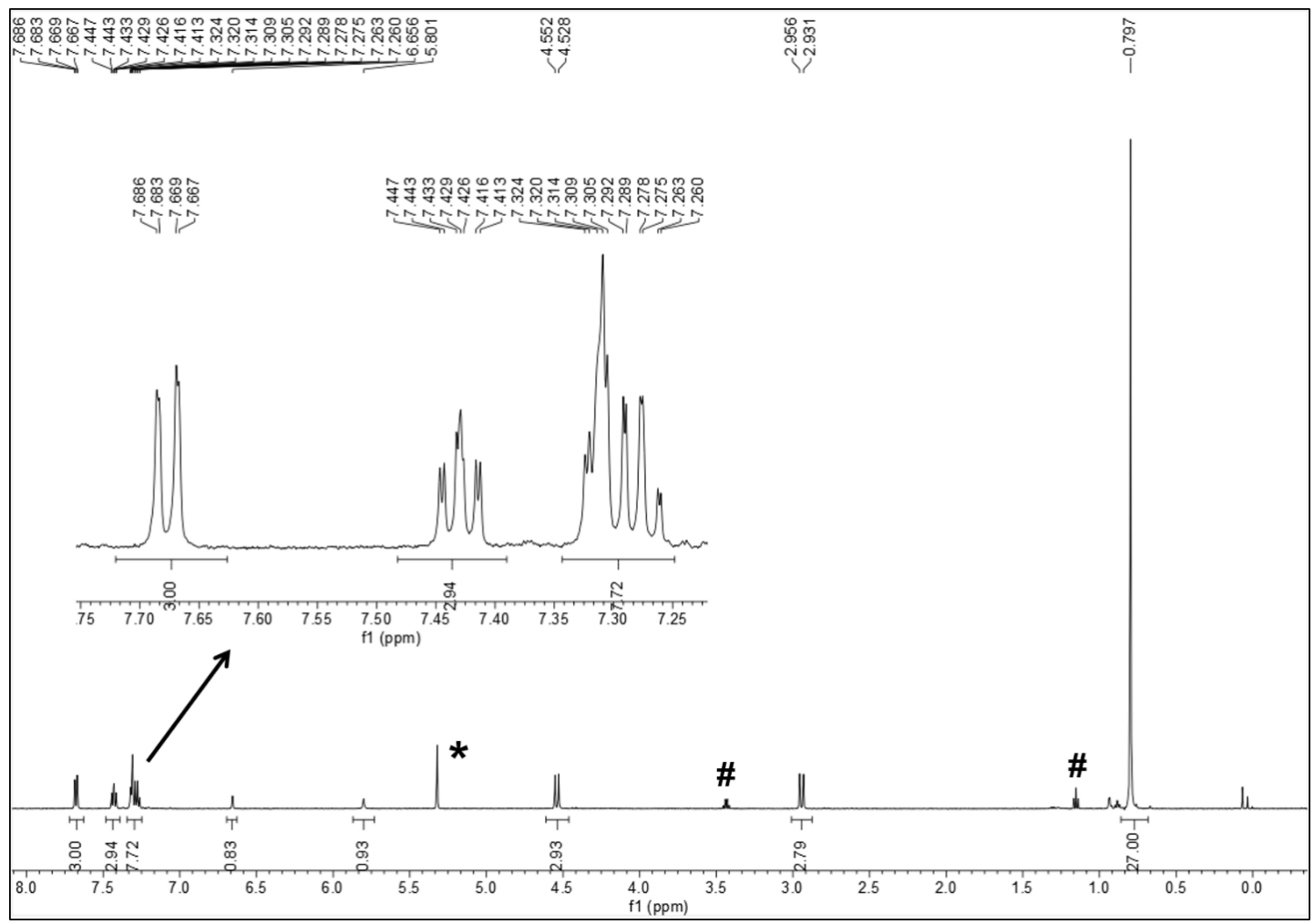

Figure S5. ${ }^{1} \mathrm{H}$ NMR spectrum of $\left[\mathrm{Th}\left(\mathrm{NHAr}^{\mathrm{F}}\right)(\operatorname{TriNOx})\right](3)$ in $\mathrm{CD}_{2} \mathrm{Cl}_{2}(*)$, \# corresponds to trace solvent signals. 


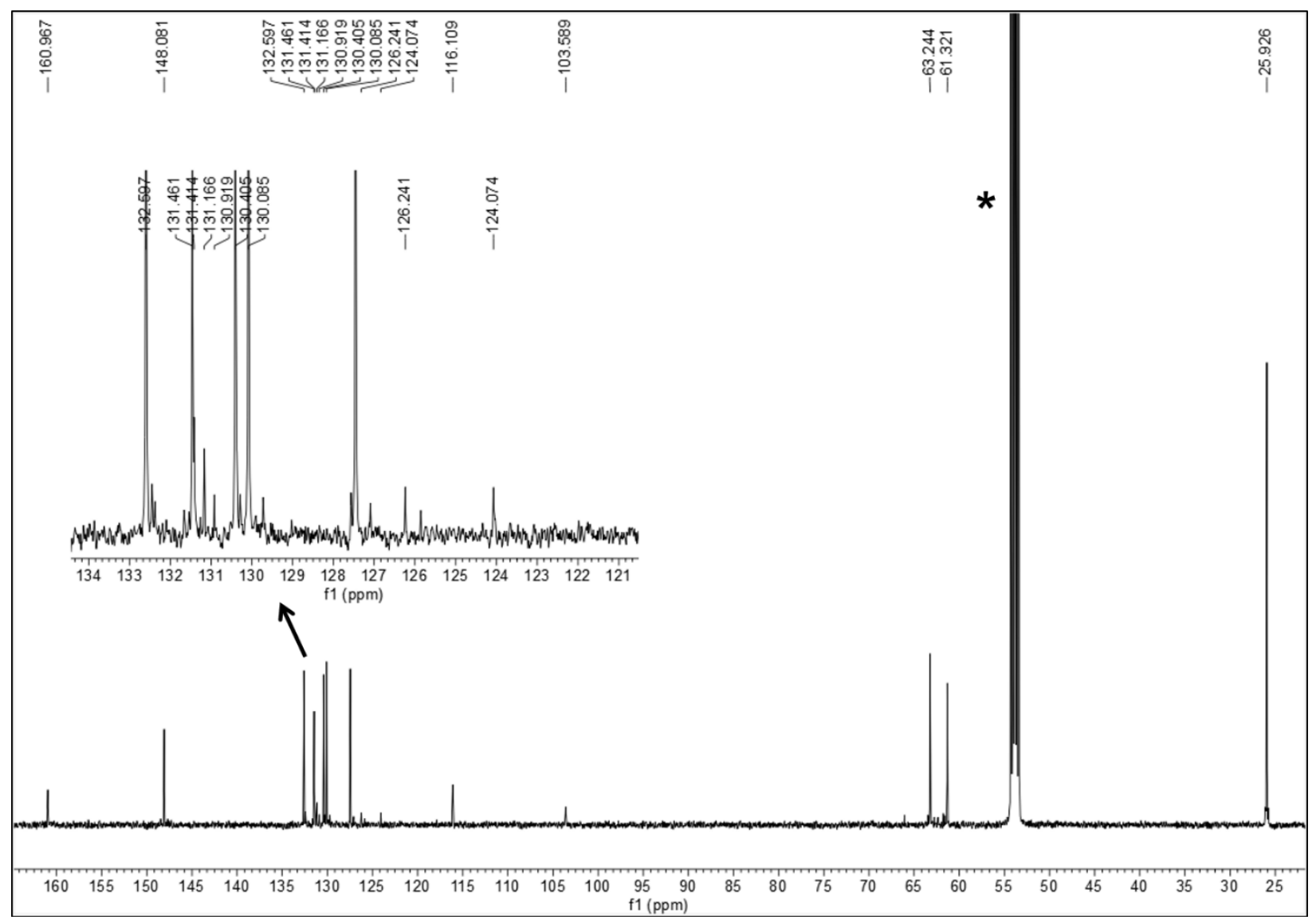

Figure S6. ${ }^{13} \mathrm{C}\left\{{ }^{1} \mathrm{H}\right\}$ NMR spectrum of $\left[\mathrm{Th}\left(\mathrm{NHAr}{ }^{\mathrm{F}}\right)(\right.$ TriNOx $\left.)\right](3)$ in $\mathrm{CD}_{2} \mathrm{Cl}_{2}(*)$.

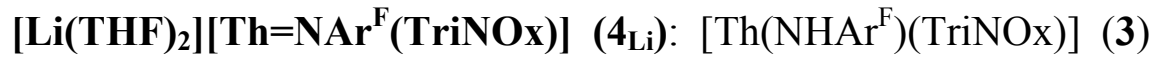
(100.1 mg, $0.10 \mathrm{mmol}, 1.0$ equiv) was suspended in THF (4 mL). With stirring, a THF solution of $\mathrm{LiCH}_{2} \mathrm{SiMe}_{3}(9.3 \mathrm{mg}, 0.10 \mathrm{mmol}$, 1.0 equiv) $(1.5 \mathrm{~mL})$ was added dropwise, producing an intense yellow color. The mixture was stirred for 10 minutes, during which time it became homogenous. The solution was filtered over Celite, and the volatiles were removed under reduced pressure. The remaining solid was washed with $n$-pentane $(2 \times 3 \mathrm{~mL})$, and the supernatant was discarded in each case. The solid was dried under

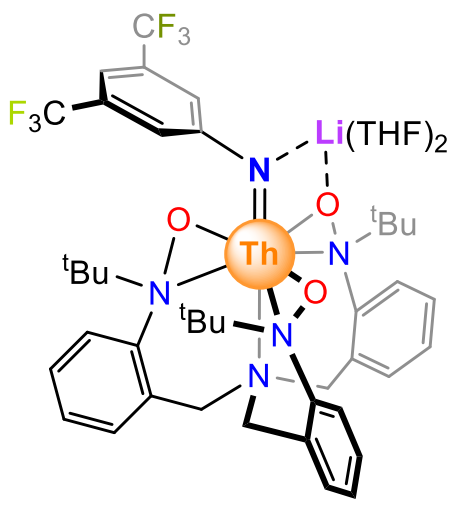
reduced pressure to afford a pale-yellow powder. Yield: $70 \mathrm{mg}, 69 \%$.

${ }^{1}$ H NMR $\left(500 \mathrm{MHz}, \mathrm{THF}-d_{8}\right): \delta 7.73\left(\mathrm{dd},{ }^{3} J_{\mathrm{HH}}=8.3 \mathrm{~Hz},{ }^{4} J_{\mathrm{HH}}=1.3 \mathrm{~Hz}, 3 \mathrm{H}, \mathrm{Ar}-\mathrm{H}\right), 7.32(\mathrm{ddd}$, $\left.{ }^{3} J_{\mathrm{HH}}=8.5 \mathrm{~Hz},{ }^{3} J_{\mathrm{HH}}=7.2 \mathrm{~Hz},{ }^{4} J_{\mathrm{HH}}=1.7 \mathrm{~Hz}, 3 \mathrm{H}, \mathrm{Ar}-\mathrm{H}\right), 7.26\left(\mathrm{dd},{ }^{3} J_{\mathrm{HH}}=7.7 \mathrm{~Hz},{ }^{4} J_{\mathrm{HH}}=1.7 \mathrm{~Hz}\right.$, $3 \mathrm{H}, \mathrm{Ar}-\mathrm{H}), 7.15\left(\mathrm{td},{ }^{3} J_{\mathrm{HH}}=7.4 \mathrm{~Hz},{ }^{4} J_{\mathrm{HH}}=1.3 \mathrm{~Hz}, 3 \mathrm{H}, \mathrm{Ar}-\mathrm{H}\right), 6.83\left(\mathrm{~d},{ }^{4} J_{\mathrm{HH}}=1.7 \mathrm{~Hz}, 2 \mathrm{H}, o-\mathrm{H}\right.$, 3,5- $\left.\left(\mathrm{CF}_{3}\right)_{2}-\mathrm{C}_{6} \mathrm{H}_{3}\right), 6.13\left(\mathrm{~s}, 1 \mathrm{H}, p-\mathrm{H}, 3,5-\left(\mathrm{CF}_{3}\right)_{2}-\mathrm{C}_{6} \mathrm{H}_{3}\right), 4.38\left(\mathrm{~d},{ }^{3} J_{\mathrm{HH}}=11.7 \mathrm{~Hz}, 3 \mathrm{H}, \mathrm{CH}_{2}\right), 2.75(\mathrm{~d}$, $\left.{ }^{3} J_{\mathrm{HH}}=11.7 \mathrm{~Hz}, 3 \mathrm{H}, \mathrm{CH}_{2}\right), 0.83\left(\mathrm{~s}, 27 \mathrm{H}, \mathrm{C}\left(\mathrm{CH}_{3}\right)_{3}\right)$. 
${ }^{13} \mathbf{C}\left\{{ }^{1} \mathbf{H}\right\}$ NMR $\left(125.66 \mathrm{MHz}, \mathrm{THF}-d_{8}\right): 150.72,133.18,132.98,130.53\left(\mathrm{q},{ }^{2} J_{\mathrm{FC}}=29.8 \mathrm{~Hz}, C-\right.$ $\left.\mathrm{CF}_{3}, 3,5-\left(\mathrm{CF}_{3}\right)_{2}-\mathrm{C}_{6} \mathrm{H}_{3}\right), 130.39,129.42,126.76\left(\mathrm{q},{ }^{1} J_{\mathrm{FC}}=272.3 \mathrm{~Hz}, C \mathrm{~F}_{3}, 3,5-\left(\mathrm{CF}_{3}\right)_{2}-\mathrm{C}_{6} \mathrm{H}_{3}\right)$, 126.72, $121.49\left(\mathrm{q},{ }^{3} J_{\mathrm{FC}}=3.6 \mathrm{~Hz}, o-\mathrm{C}, 3,5-\left(\mathrm{CF}_{3}\right)_{2}-\mathrm{C}_{6} \mathrm{H}_{3}\right), 98.43\left(\mathrm{~m}, p-\mathrm{C}, 3,5-\left(\mathrm{CF}_{3}\right)_{2}-\mathrm{C}_{6} \mathrm{H}_{3}\right), 62.62$

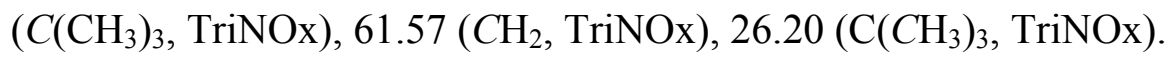

${ }^{19} \mathbf{F}\left\{{ }^{1} \mathbf{H}\right\}$ NMR $\left(376.30 \mathrm{MHz}, \mathrm{THF}-d_{8}\right): \delta-63.28(\mathrm{~s})$.

${ }^{7}$ Li NMR (155.45 MHz, THF- $\left.d_{8}\right): \delta 0.51$.

Anal. Calc. for $\mathbf{C}_{49} \mathbf{H}_{64} \mathbf{F}_{6} \mathbf{L i N}_{5} \mathbf{O}_{5} \mathbf{T h}$ : C, 50.91; H, 5.58; N, 6.06. Found: C, 51.00; H, 5.52; N, 6.07 .

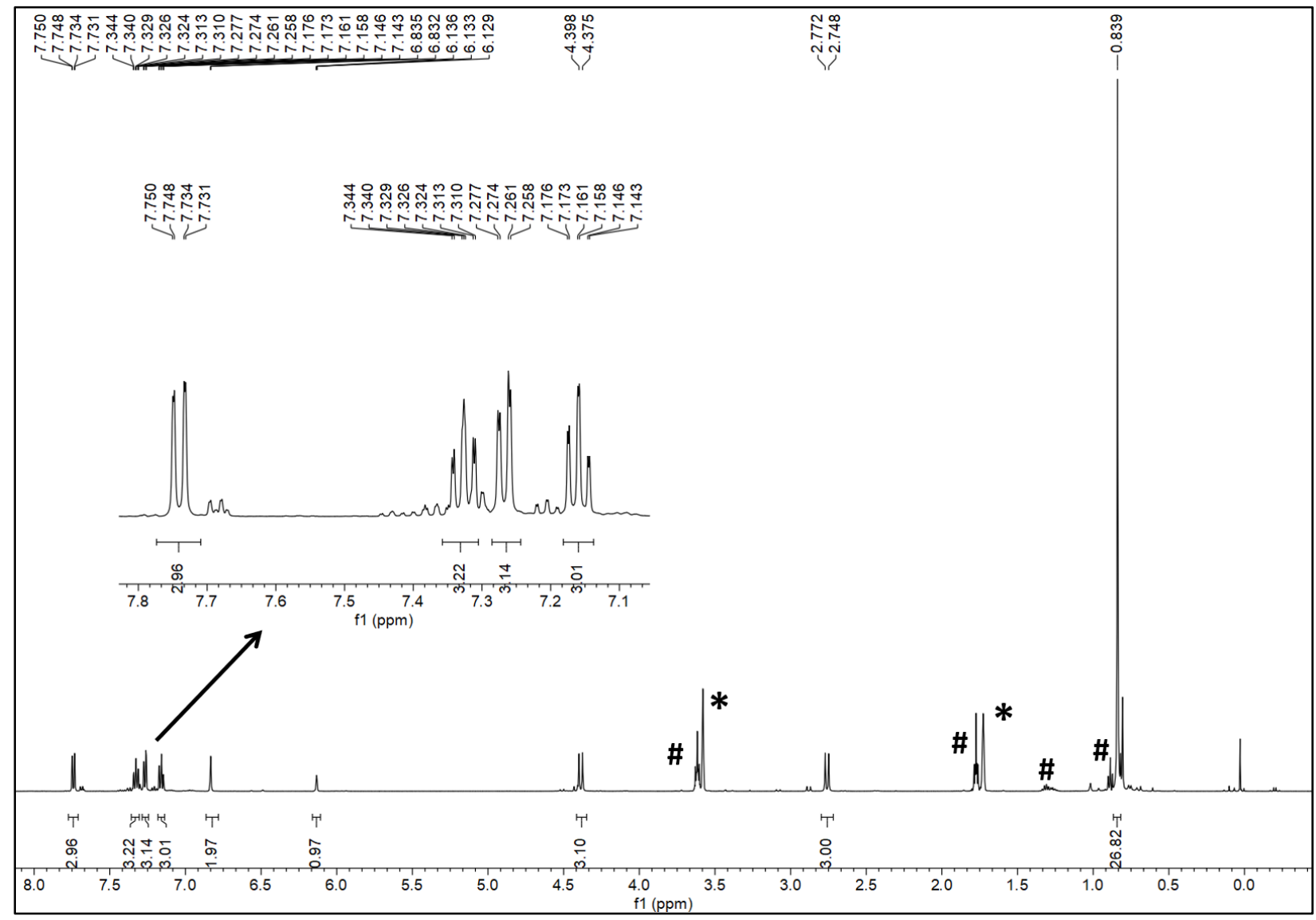

Figure S7. ${ }^{1} \mathrm{H}$ NMR spectrum of $\left[\mathrm{Li}(\mathrm{THF})_{2}\right]\left[\mathrm{Th}=\mathrm{NAr}^{\mathrm{F}}(\operatorname{TriNOx})\right]\left(\mathbf{4}_{\mathrm{Li}}\right)$ in $\mathrm{THF}-d_{8}(*)$, \# corresponds to trace solvent signals. 


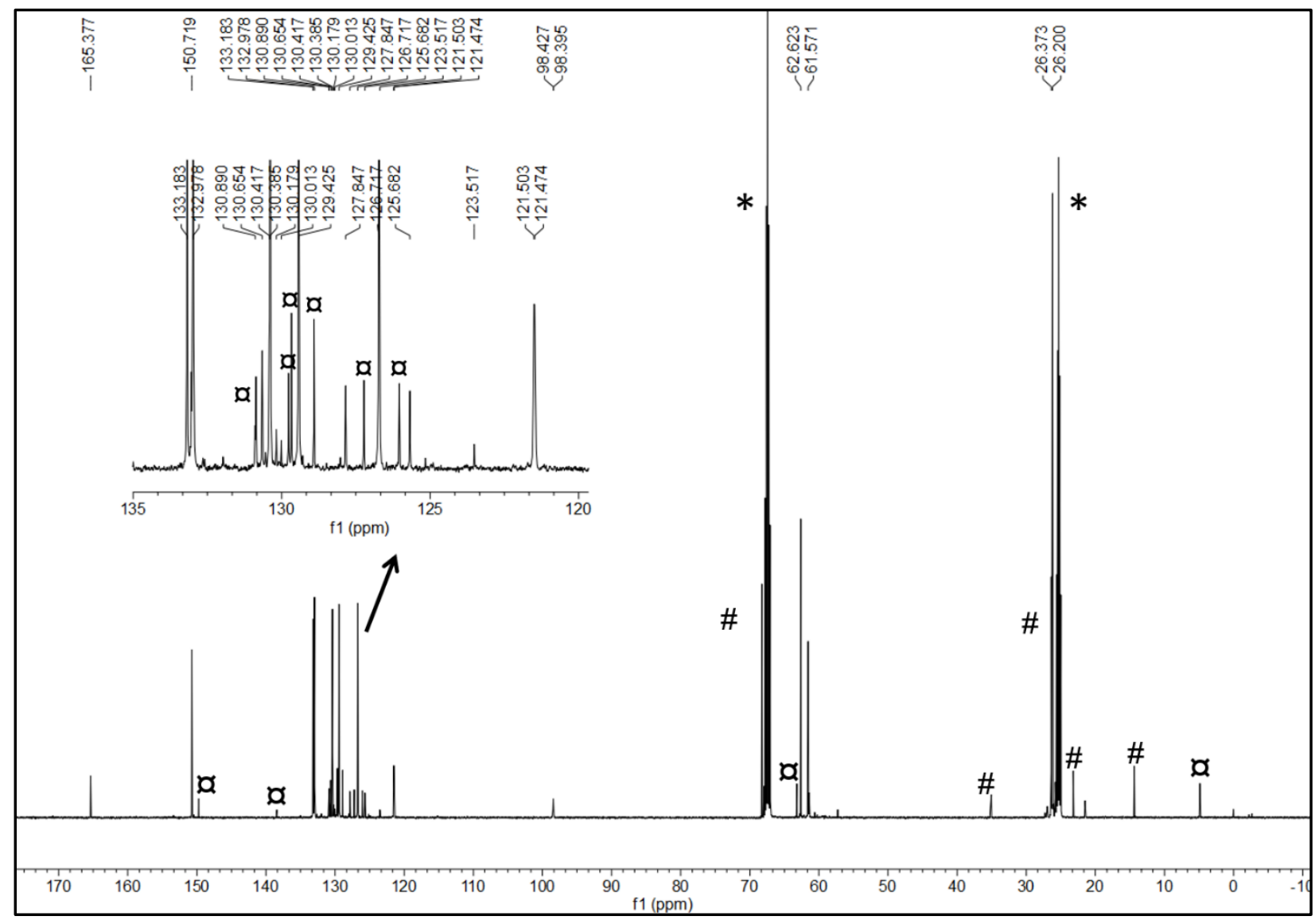

Figure S8. ${ }^{13} \mathrm{C}\left\{{ }^{1} \mathrm{H}\right\}$ NMR spectrum of $\left[\mathrm{Li}(\mathrm{THF})_{2}\right]\left[\mathrm{Th}=\mathrm{NAr}{ }^{\mathrm{F}}(\operatorname{TriNOx})\right]\left(\mathbf{4}_{\mathrm{Li}}\right)$ in $\mathrm{THF}-d_{8}(*)$, \# corresponds to trace solvent signals, $x$ corresponds to decomposition products.
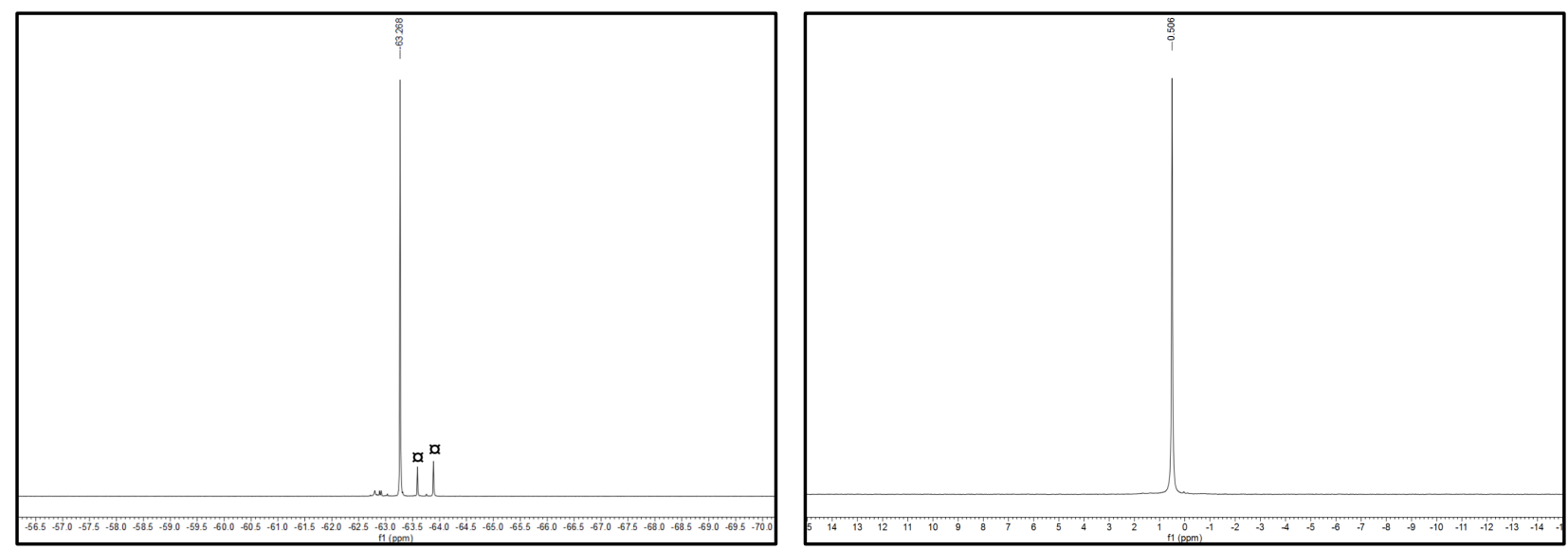

Figure S9. ${ }^{19} \mathrm{~F}\left\{{ }^{1} \mathrm{H}\right\}$ (left) and ${ }^{7} \mathrm{Li}$ (right) NMR spectra of $\left[\mathrm{Li}(\mathrm{THF})_{2}\right]\left[\mathrm{Th}=\mathrm{NAr}{ }^{\mathrm{F}}(\operatorname{TriNOx})\right]\left(\mathbf{4}_{\mathrm{Li}}\right)$ in THF- $d_{8}$, a corresponds to decomposition products. 
[K(2.2.2-cryptand)][Th $=N$ NAr $^{\mathrm{F}}$ (TriNOx) $]\left(\mathbf{4}_{\varnothing)}\right.$ :

[Th(NHAr $\left.{ }^{\mathrm{F}}\right)($ TriNOx)] (3) $(75 \mathrm{mg}, 75 \mu \mathrm{mol}, 1.0$ equiv) and 2.2.2-cryptand ( $28 \mathrm{mg}, 75 \mu \mathrm{mol}, 1.0$ equiv) were mixed in the solid phase and suspended in a 1:1 solution of THF:toluene (4 $\mathrm{mL})$. With stirring, a THF $(2 \mathrm{~mL})$ solution of KBn $(10 \mathrm{mg}, 75$ $\mu$ mol, 1.0 equiv) was added slowly dropwise, producing gradually a clear solution. Close to the end of the addition, a yellow precipitate formed. THF $(2 \mathrm{~mL})$ was added into the reaction mixture, and the suspension was stirred for 10 minutes before being chilled at $-21{ }^{\circ} \mathrm{C}$ for crystallization. The supernatant was discarded, and the remaining solid was washed with $n$-pentane $(3 \times 2 \mathrm{~mL})$ before being dried under

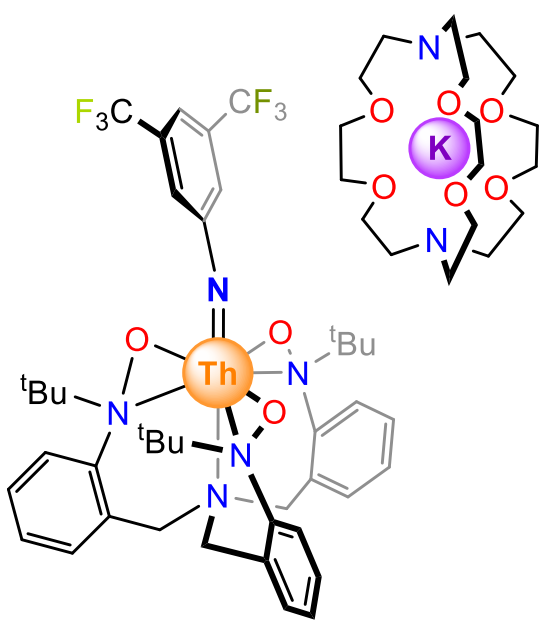
reduced pressure to afford a yellow solid. Yield: $83 \mathrm{mg}, 78 \%$.

${ }^{1}$ H NMR $\left(400 \mathrm{MHz}, \mathrm{THF}-d_{8}\right): \delta 7.82\left(\mathrm{~d},{ }^{3} J_{\mathrm{HH}}=8.2 \mathrm{~Hz}, 3 \mathrm{H}, \mathrm{Ar}-\mathrm{H}\right), 7.19\left(\mathrm{t},{ }^{3} J_{\mathrm{HH}}=7.6 \mathrm{~Hz}, 3 \mathrm{H}\right.$, Ar-H), $7.10\left(\mathrm{~d},{ }^{3} J_{\mathrm{HH}}=7.6 \mathrm{~Hz}, 3 \mathrm{H}, \mathrm{Ar}-\mathrm{H}\right), 6.99\left(\mathrm{t},{ }^{3} J_{\mathrm{HH}}=7.4 \mathrm{~Hz}, 3 \mathrm{H}, \mathrm{Ar}-\mathrm{H}\right), 6.40(\mathrm{~s}, 2 \mathrm{H}, o-\mathrm{H}$, 3,5- $\left.\left(\mathrm{CF}_{3}\right)_{2}-\mathrm{C}_{6} \mathrm{H}_{3}\right), 5.77\left(\mathrm{~s}, 1 \mathrm{H}, p-\mathrm{H}, 3,5-\left(\mathrm{CF}_{3}\right)_{2}-\mathrm{C}_{6} \mathrm{H}_{3}\right), 4.19\left(\mathrm{~d},{ }^{2} J_{\mathrm{HH}}=11.4 \mathrm{~Hz}, 3 \mathrm{H}, \mathrm{CH}_{2}\right.$, TriNOx), 3.60 (s, 12H, 2.2.2-cryptand), $3.54\left(\mathrm{~m}, 12 \mathrm{H}, 2.2 .2\right.$-cryptand), $2.53\left(\mathrm{t},{ }^{3} J_{\mathrm{HH}}=4.7 \mathrm{~Hz}\right.$, $12 \mathrm{H}, 2.2 .2$-cryptand), $2.44\left(\mathrm{~d},{ }^{2} J_{\mathrm{HH}}=11.4 \mathrm{~Hz}, 3 \mathrm{H}, \mathrm{CH}_{2}\right.$, TriNOx), $0.87\left(\mathrm{~s}, 27 \mathrm{H}, \mathrm{C}\left(\mathrm{CH}_{3}\right)_{3}\right)$.

${ }^{13} \mathbf{C}\left\{{ }^{1} \mathbf{H}\right\}$ NMR $\left(125.66 \mathrm{MHz}, \mathrm{THF}-d_{8}\right): \delta 152.65,133.81,132.16,130.91,129.86\left(\mathrm{q},{ }^{2} J_{\mathrm{FC}}=29.3\right.$ $\left.\mathrm{Hz}, C-\mathrm{CF}_{3}, 3,5-\left(\mathrm{CF}_{3}\right)_{2}-\mathrm{C}_{6} \mathrm{H}_{3}\right), 128.14,127.0\left(\mathrm{q},{ }^{1} J_{\mathrm{FC}}=272.6 \mathrm{~Hz}, C \mathrm{~F}_{3}, 3,5-\left(\mathrm{CF}_{3}\right)_{2}-\mathrm{C}_{6} \mathrm{H}_{3}\right), 123.64$ (s, $\left.o-\mathrm{C}, 3,5-\left(\mathrm{CF}_{3}\right)_{2}-\mathrm{C}_{6} \mathrm{H}_{3}\right), 93.06$ (m, $\left.p-\mathrm{C}, 3,5-\left(\mathrm{CF}_{3}\right)_{2}-\mathrm{C}_{6} \mathrm{H}_{3}\right), 71.34$ (2.2.2-cryptand), 68.52 (2.2.2cryptand), $62.53\left(\mathrm{CH}_{2}\right.$, TriNOx), $62.31\left(\mathrm{C}\left(\mathrm{CH}_{3}\right)_{3}\right.$, TriNOx $), 54.91$ (2.2.2-cryptand), 26.38 $\left(\mathrm{C}\left(\mathrm{CH}_{3}\right)_{3}\right.$, TriNOx $)$.

${ }^{19} \mathbf{F}\left\{{ }^{1} \mathbf{H}\right\}$ NMR $\left(376.30 \mathrm{MHz}, \mathrm{THF}-d_{8}\right): \delta-62.94$.

Anal. Calc. for $\mathbf{C}_{59} \mathbf{H}_{84} \mathbf{F}_{6} \mathbf{K N}_{7} \mathbf{O}_{9} \mathbf{T h}: \mathrm{C}$, 49.89; H, 5.96; N, 6.90. Found: C, 49.73; H, 6.00; N, 6.82 . 


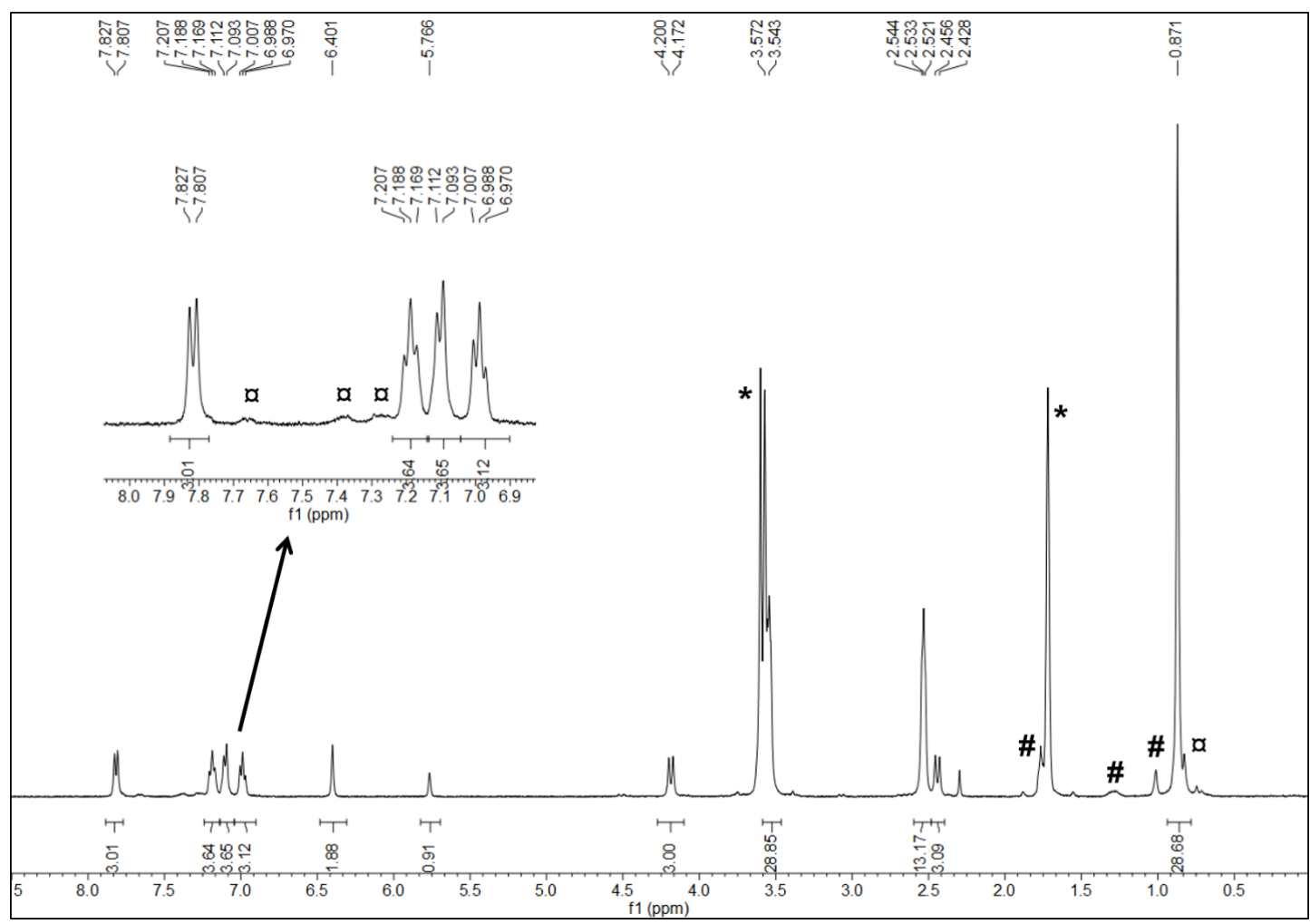

Figure S10. ${ }^{1} \mathrm{H}$ NMR spectrum of $[\mathrm{K}(2.2 .2$-cryptand $)]\left[\mathrm{Th}=\mathrm{NAr}{ }^{\mathrm{F}}(\operatorname{TriNOx})\right]\left(\mathbf{4}_{\varnothing}\right)$ in $\mathrm{THF}-d_{8}(*)$, \# corresponds to trace solvent signals, $\alpha$ corresponds to decomposition products.

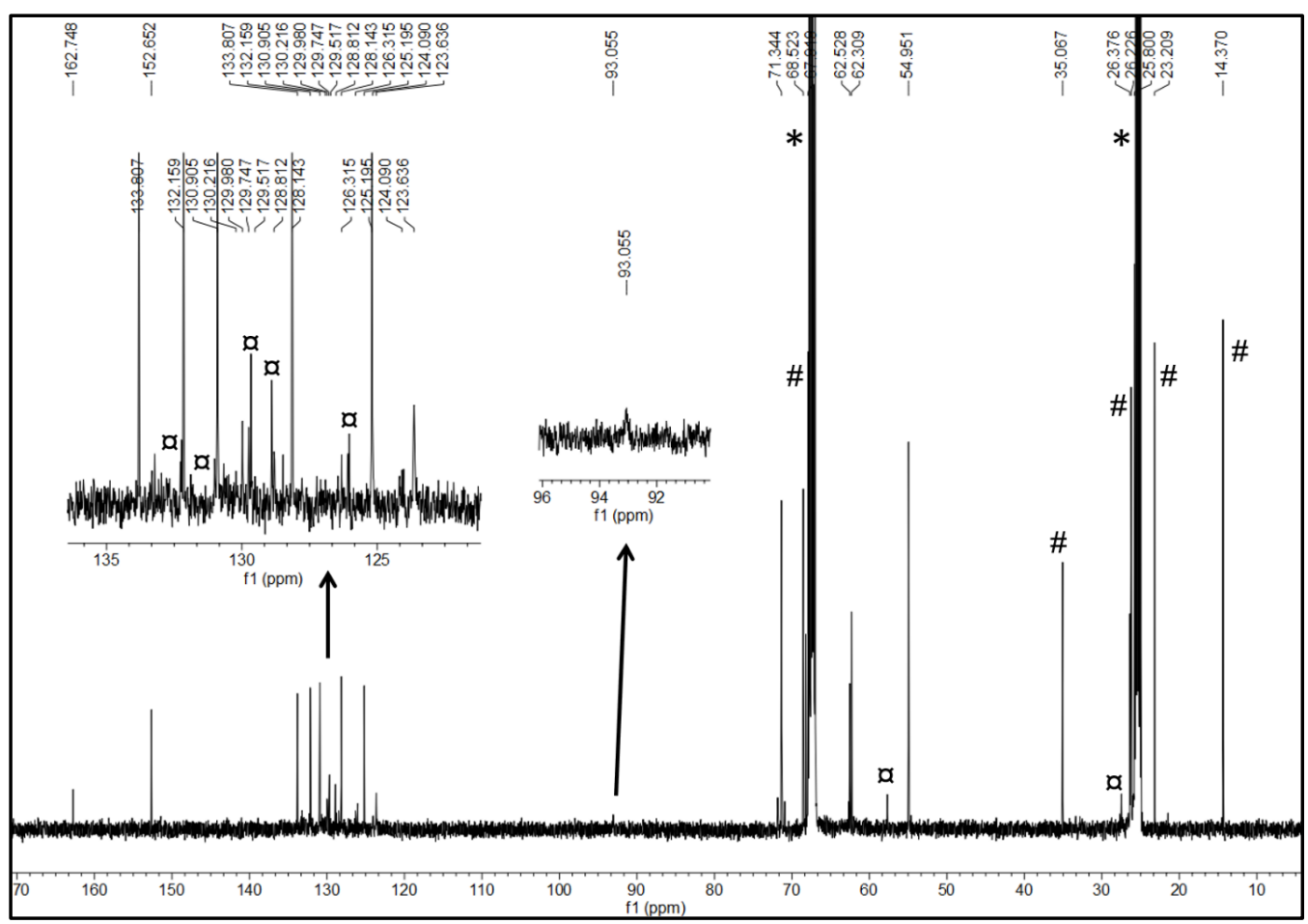

Figure S11. ${ }^{13} \mathrm{C}\left\{{ }^{1} \mathrm{H}\right\}$ NMR spectrum of $[\mathrm{K}(2.2$.2-cryptand $)]\left[\mathrm{Th}=\mathrm{NAr}{ }^{\mathrm{F}}(\operatorname{TriNOx})\right]\left(\mathbf{4}_{\varnothing}\right)$ in $\mathrm{THF}-d_{8}$ $(*)$, \# corresponds to trace solvent signals, $\propto$ corresponds to decomposition products. 


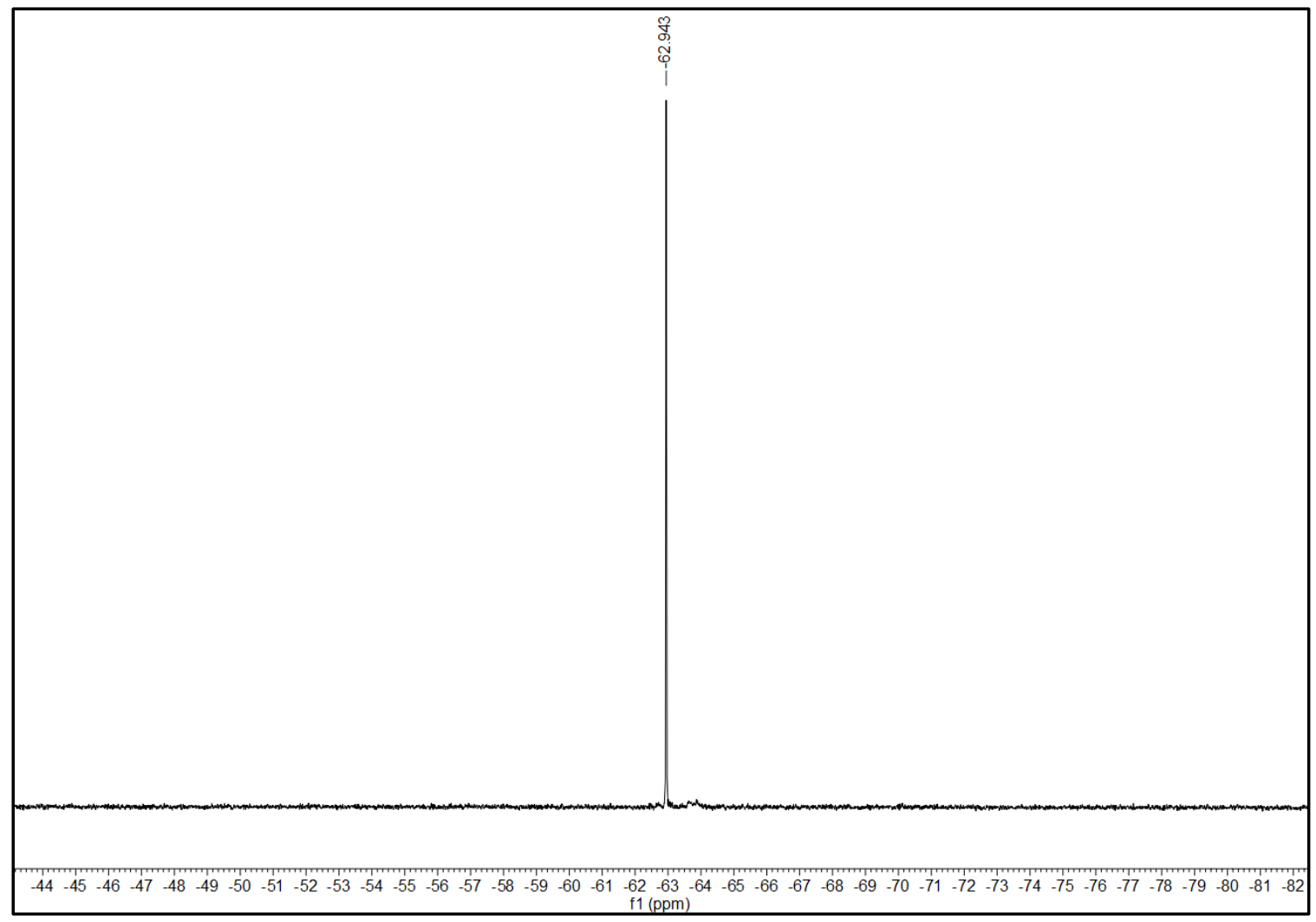

Figure S12. ${ }^{19} \mathrm{~F}\left\{{ }^{1} \mathrm{H}\right\}$ NMR spectrum of $[\mathrm{K}(2.2 .2$-cryptand $)]\left[\mathrm{Th}=\mathrm{NAr}{ }^{\mathrm{F}}(\operatorname{TriNOx})\right]\left(\mathbf{4}_{\varnothing}\right)$ in $\mathrm{THF}-d_{8}$.

[Ce(THF)(TriNOx)] $\left[\mathrm{BAr}_{4}^{\mathrm{F}}\right]\left(\mathbf{6}_{\mathrm{Ce}}\right)$ :

$\mathbf{6}_{\mathrm{Ce}}$ was previously synthesized by oxidation of $[\mathrm{Ce}(\mathrm{THF})(\mathrm{TriNOx})](7)$ by $\mathrm{Fc}\left[\mathrm{BAr}_{4}^{\mathrm{F}}{ }_{4}{ }^{6}\right.$ An alternative procedure was employed here. [CeCl(TriNOx)] (150 mg, $0.21 \mathrm{mmol}, 1.0$ equiv) and $\mathrm{NaBAr}_{4}^{\mathrm{F}}(185 \mathrm{mg}, 0.21 \mathrm{mmol}, 1.0$ equiv) were dissolved in $\mathrm{CH}_{2} \mathrm{Cl}_{2}(20 \mathrm{~mL})$ and stirred overnight at room temperature. The volatiles were evaporated and the solid was

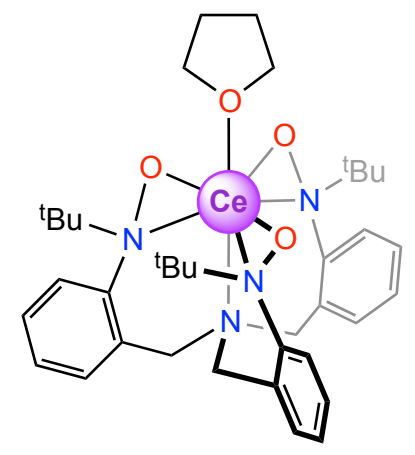<smiles>FC(F)(F)c1cc([B-](c2cc(C(F)(F)F)cc(C(F)(F)F)c2)(c2cc(C(F)(F)F)cc(C(F)(F)F)c2)c2cc(C(F)(F)F)cc(C(F)(F)F)c2)cc(C(F)(F)F)c1</smiles>
extracted with a mixture of toluene $(10 \mathrm{~mL})$ and THF $(1 \mathrm{~mL})$ and filtered to remove the $\mathrm{NaCl}$. The filtrate was layered with $n$-pentane $(10 \mathrm{~mL})$ and cooled to $-20^{\circ} \mathrm{C}$ for 3 days to yield dark red crystals that were collected and washed with $n$-pentane $(2 \times 2 \mathrm{~mL})$ before being dried under reduced pressure. Yield: $215 \mathrm{mg}, 67 \%$. 
$\left[\operatorname{Th}\left(\mathrm{OEt}_{2}\right)(\operatorname{TriNOx})\right]\left[\mathrm{BAr}^{\mathrm{F}}{ }_{4}\right] \quad\left(\mathbf{6}_{\mathrm{Th}}\right)$ : [ThCl(TriNOx)] (1) (656 mg, 0.81 mmol, 1.0 equiv) was dissolved in DCM $(10 \mathrm{~mL})$. With stirring, an $\mathrm{Et}_{2} \mathrm{O}$ solution $(8 \mathrm{~mL})$ of $\mathrm{NaBAr}_{4}^{\mathrm{F}}(715 \mathrm{mg}$, $0.81 \mathrm{mmol}, 1.0$ equiv) was added dropwise, forming a pale-yellow solution. The reaction was stirred for
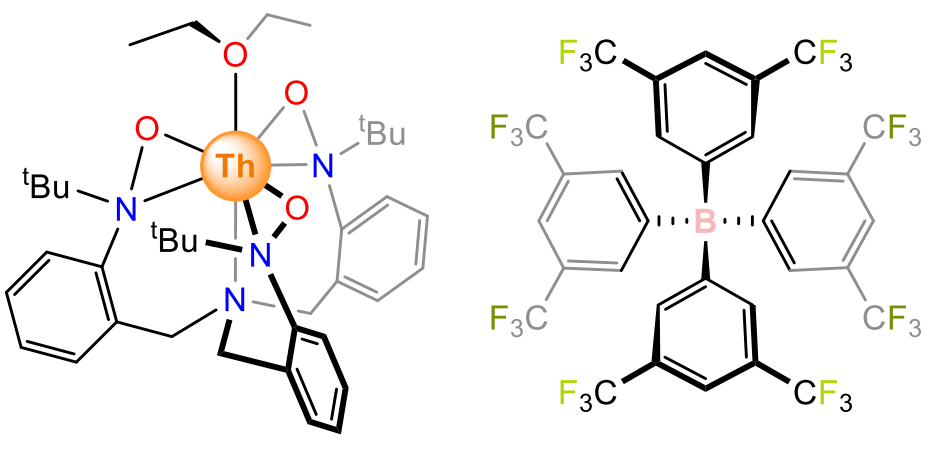
48 hours, after which time the solution was filtered over a Celite plug, and the solvent was removed under reduced pressure. The product was extracted with $\mathrm{Et}_{2} \mathrm{O}$, and then allowed to crystallize from an $\mathrm{Et}_{2} \mathrm{O} / n$-pentane layering. Yield: $560 \mathrm{mg}, 40 \%$.

${ }^{1}$ H NMR $\left(500 \mathrm{MHz}, \mathrm{THF}-d_{8}\right): \delta$ 7.83-7.74 (m, 8H, $\left.o-\mathrm{H}, \mathrm{BAr}{ }^{\mathrm{F}}\right), 7.64-7.48\left(\mathrm{~m}, 13 \mathrm{H}, p-\mathrm{H}\left(\mathrm{BAr}^{\mathrm{F}}\right)+\right.$ Ar-H (TriNOx)), $7.43\left(\mathrm{t},{ }^{3} J_{\mathrm{HH}}=7.5 \mathrm{~Hz}, 3 \mathrm{H}, \mathrm{Ar}-\mathrm{H}, \operatorname{TriNOx}\right), 4.69\left(\mathrm{~d},{ }^{2} J_{\mathrm{HH}}=12.5 \mathrm{~Hz}, 3 \mathrm{H}, \mathrm{CH}_{2}\right)$, $3.44\left(\mathrm{~d},{ }^{2} J_{\mathrm{HH}}=12.6 \mathrm{~Hz}, 3 \mathrm{H}, \mathrm{CH}_{2}\right), 3.38\left(\mathrm{q},{ }^{3} J_{\mathrm{HH}}=7.0 \mathrm{~Hz}, 4 \mathrm{H}, \mathrm{Et}_{2} \mathrm{O}\right), 1.11\left(\mathrm{t},{ }^{3} J_{\mathrm{HH}}=7.0 \mathrm{~Hz}, 6 \mathrm{H}\right.$, $\left.\mathrm{Et}_{2} \mathrm{O}\right), 0.77$ (s, $\left.27 \mathrm{H}, \mathrm{C}\left(\mathrm{CH}_{3}\right)_{3}\right)$.

${ }^{13} \mathbf{C}\left\{{ }^{1} \mathbf{H}\right\}$ NMR $\left(125.66 \mathrm{MHz}, \mathrm{THF}-d_{8}\right): \delta 162.42\left(\mathrm{q},{ }^{1} J_{\mathrm{BC}}=49.5 \mathrm{~Hz}, \mathrm{~B}-C_{i p s o}, \mathrm{BAr}{ }^{\mathrm{F}}\right), 146.84$, $136.11-135.33\left(\mathrm{~m}, o-\mathrm{C}, \mathrm{BAr}{ }^{\mathrm{F}}\right), 134.12,131.63,131.58,131.04,130.03\left(\mathrm{qq},{ }^{3} J_{\mathrm{FC}}=31.4 \mathrm{~Hz},{ }^{2} J_{\mathrm{BC}}\right.$ $\left.=2.8 \mathrm{~Hz}, o-\mathrm{C}, \mathrm{BAr}^{\mathrm{F}}\right), 129.48,125.52\left(\mathrm{q},{ }^{1} J_{\mathrm{FC}}=272.2 \mathrm{~Hz}, C \mathrm{~F}_{3}\right), 118.16\left(\mathrm{~m}, p-\mathrm{C}, \mathrm{BAr}^{\mathrm{F}}\right), 66.30$ $\left(\mathrm{CH}_{3}, \mathrm{Et}_{2} \mathrm{O}\right), 64.16\left(\mathrm{C}\left(\mathrm{CH}_{3}\right)_{3}\right.$, TriNOx $), 60.59\left(\mathrm{CH}_{2}\right.$, TriNOx $), 26.24\left(\mathrm{C}\left(\mathrm{CH}_{3}\right)_{3}\right.$, TriNOx $), 15.65$ $\left(\mathrm{CH}_{2}, \mathrm{Et}_{2} \mathrm{O}\right)$.

${ }^{19} \mathbf{F}\left\{{ }^{1} \mathbf{H}\right\}$ NMR $\left(376.30 \mathrm{MHz}, \mathrm{THF}-d_{8}\right): \delta-63.42$.

${ }^{11} \mathbf{B}\left\{{ }^{1} \mathbf{H}\right\}$ NMR $\left(128.35 \mathrm{MHz}, \mathrm{THF}-d_{8}\right): \delta-7.01$.

Anal. Calc. for $\mathbf{C}_{41} \mathbf{H}_{49} \mathbf{F}_{6} \mathbf{N}_{5} \mathbf{O}_{3} \mathbf{T h}$ : C, 48.32; H, 3.94; N, 3.27. Found: C, 48.71; H, 3.22; N, 3.06. 


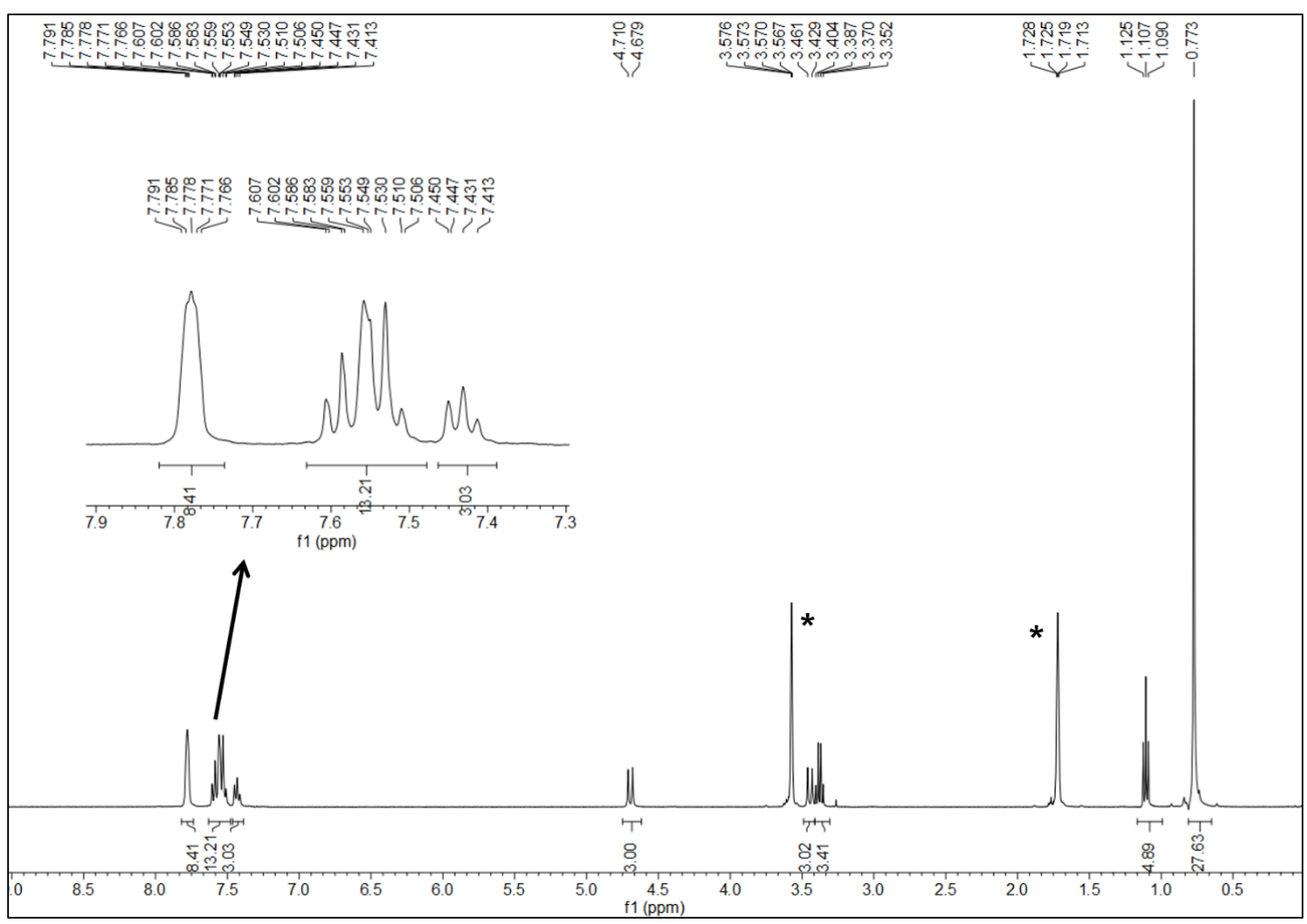

Figure S13. ${ }^{1} \mathrm{H}$ NMR spectrum of $\left[\mathrm{Th}\left(\mathrm{OEt}_{2}\right)(\operatorname{TriNOx})\right]\left[\mathrm{BAr}^{\mathrm{F}}{ }_{4}\right]\left(\mathbf{6}_{\mathrm{Th}}\right)$ in THF- $d_{8}(*)$, corresponds to trace solvent signals.

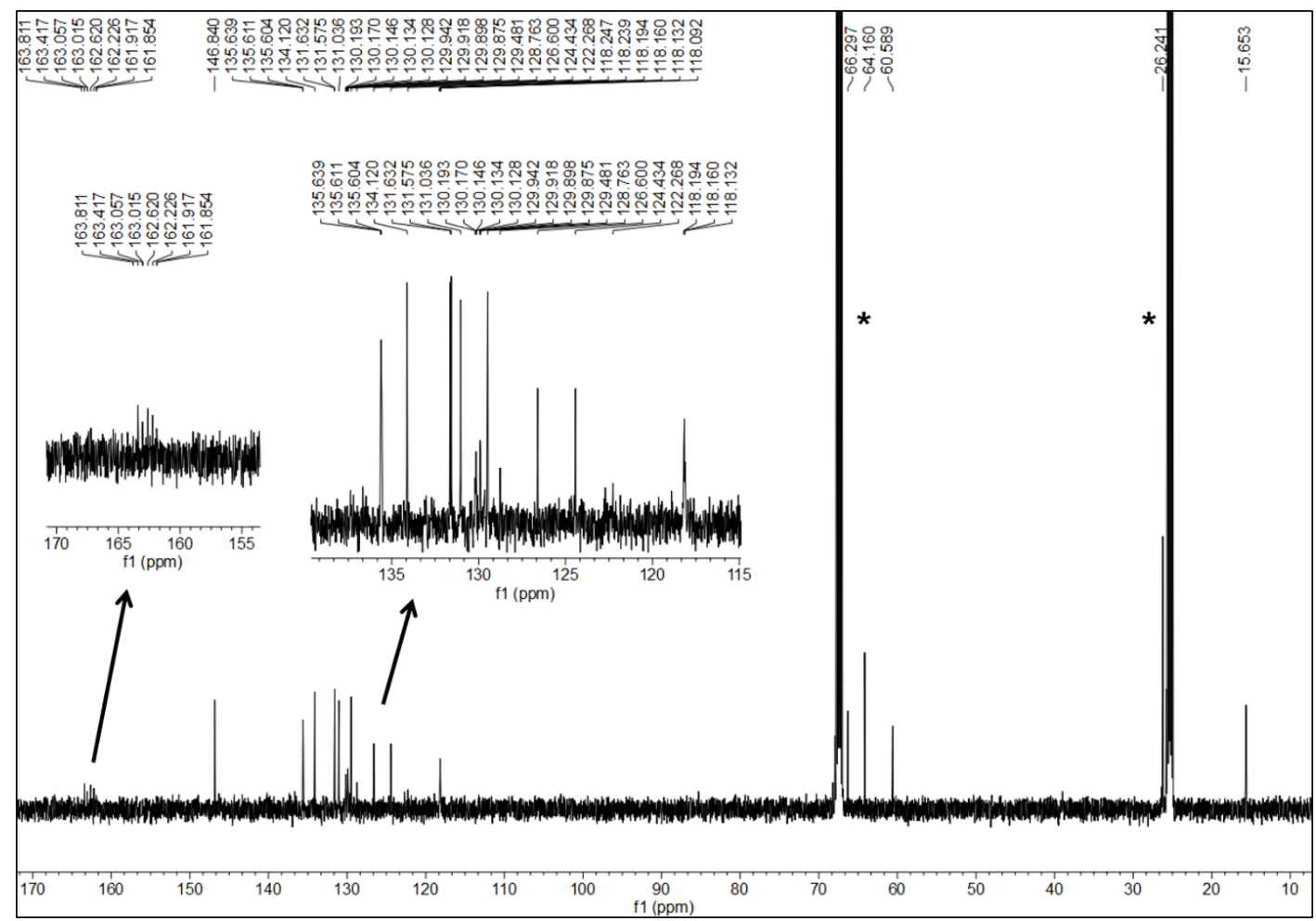

Figure S14. ${ }^{13} \mathrm{C}\left\{{ }^{1} \mathrm{H}\right\}$ NMR spectrum of $\left[\mathrm{Th}\left(\mathrm{OEt}_{2}\right)(\operatorname{TriNOx})\right]\left[\mathrm{BAr}{ }_{4}^{\mathrm{F}}\right]\left(\mathbf{6}_{\mathrm{Th}}\right)$ in $\mathrm{THF}-d_{8}\left({ }^{*}\right)$, \# corresponds to trace solvent signals. 


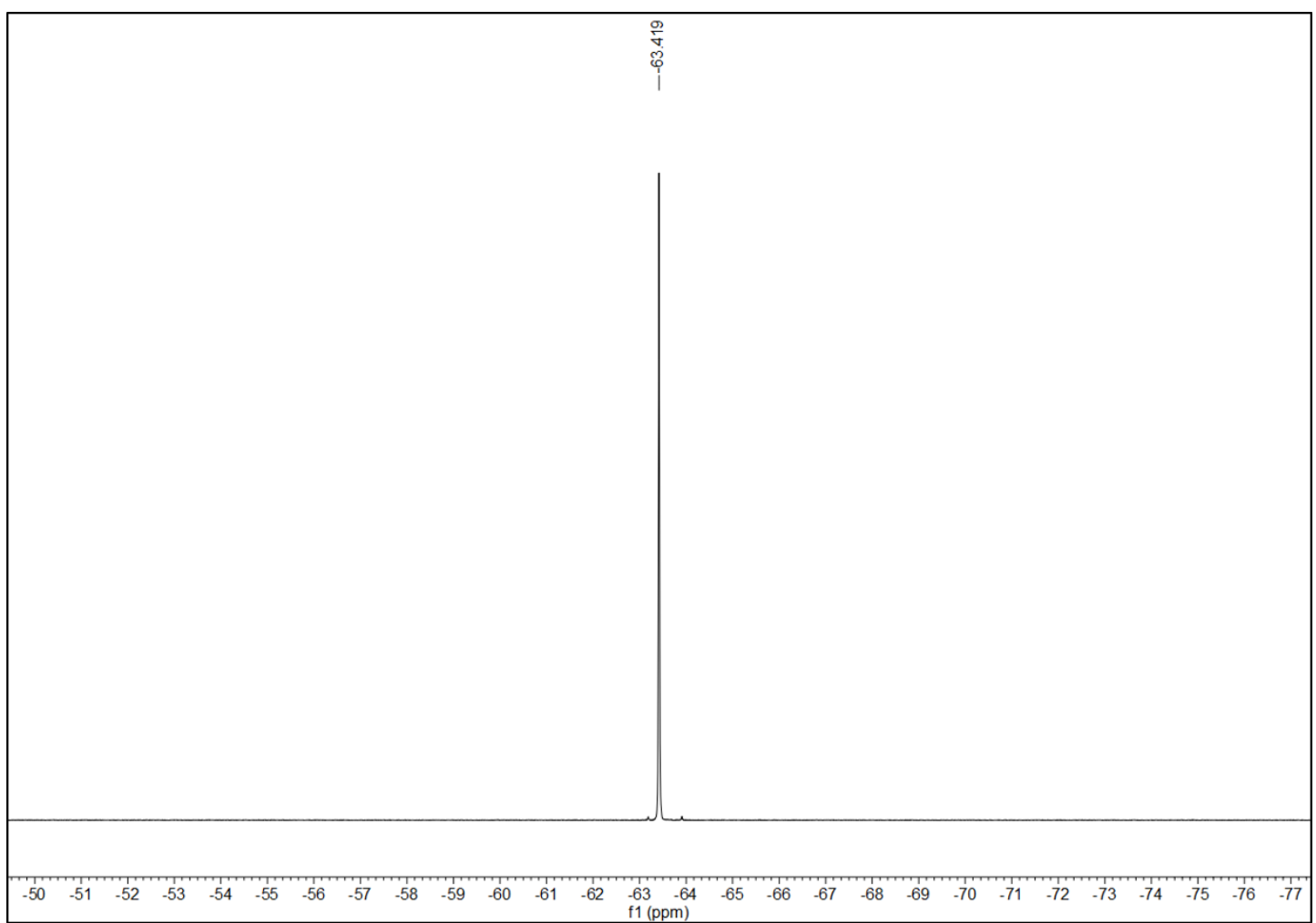

Figure S15. ${ }^{19} \mathrm{~F}\left\{{ }^{1} \mathrm{H}\right\}$ NMR spectrum of $\left[\mathrm{Th}\left(\mathrm{OEt}_{2}\right)(\operatorname{TriNOx})\left[\mathrm{BAr}_{4}^{\mathrm{F}}\right]\left(\mathbf{6}_{\mathbf{T h}}\right)\right.$ in THF- $d_{8}$.

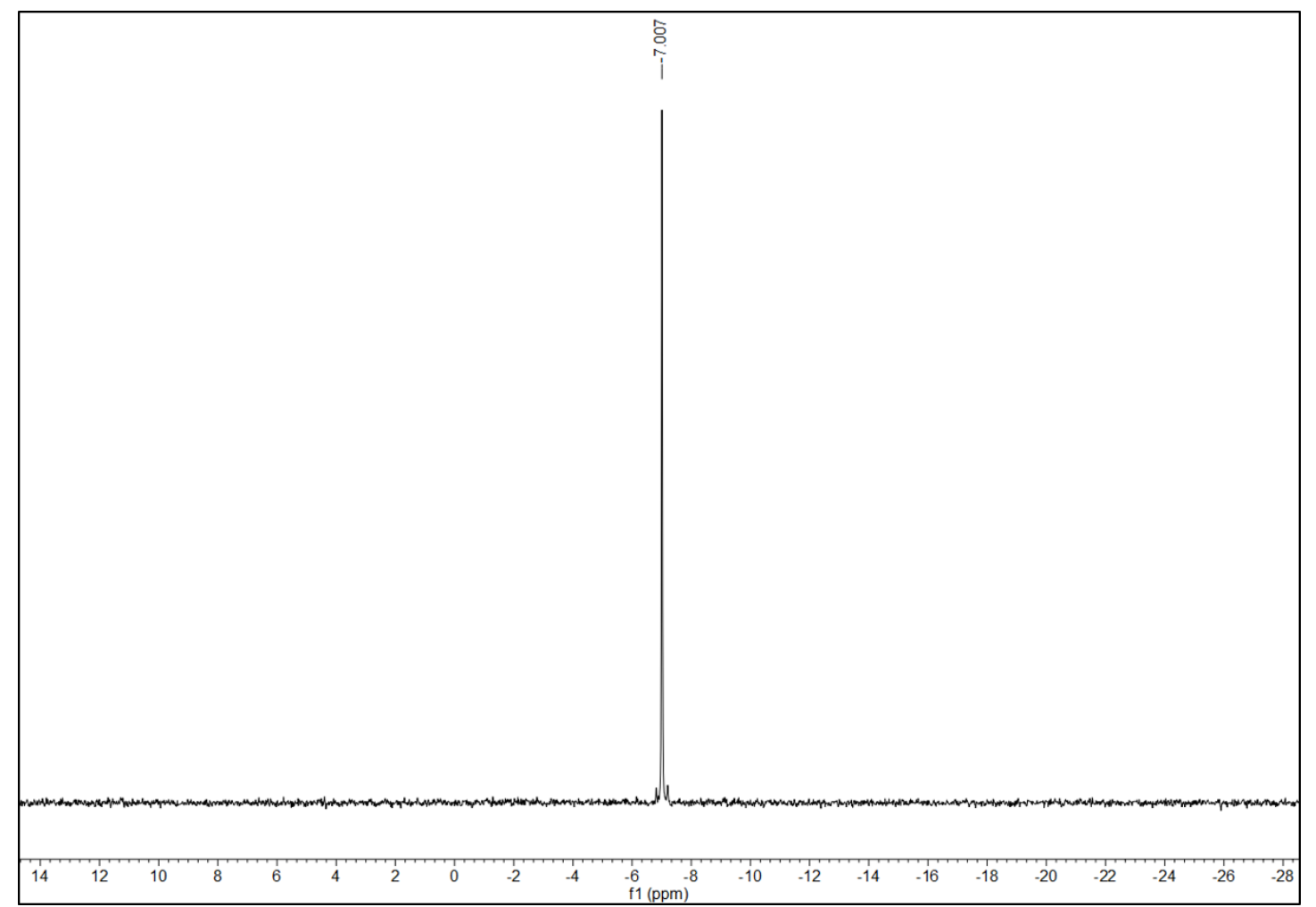

Figure S16. ${ }^{11} \mathrm{~B}\left\{{ }^{1} \mathrm{H}\right\}$ (right) NMR spectrum of $\left[\mathrm{Th}\left(\mathrm{OEt}_{2}\right)(\mathrm{TriNOx})\right]\left[\mathrm{BAr}^{\mathrm{F}}{ }_{4}\right]\left(\mathbf{6}_{\mathbf{T h}}\right)$ in $\mathrm{THF}-d_{8}$. 
X-ray Crystallography. X-ray intensity data were collected on a Bruker APEXII ${ }^{7}$ CCD area detector or a Bruker APEXIII ${ }^{8}$ D8QUEST CMOS area detector, both employing graphitemonochromated Mo- $K_{\alpha}$ radiation $(\lambda=0.71073 \AA)$ at $100(1) \mathrm{K}$. Rotation frames were integrated using SAINT, ${ }^{9}$ producing a listing of unaveraged $F^{2}$ and $\sigma\left(F^{2}\right)$ values which were then passed to the SHELXT program package ${ }^{10}$ for further processing and structure solution. The intensity data were corrected for Lorentz and polarization effects and for absorption using SADABS or TWINABS. $^{11}$ Refinement was performed by full-matrix least squares based on $F^{2}$ using SHELXL-2015. ${ }^{12}$ All of the reflections were used during refinement. Non-hydrogen atoms were refined anisotropically and hydrogen atoms were refined using a riding model. Crystallographic parameters for the studied compounds are summarized on Tables S1 and S2. 
Table S1. Crystallographic data for 1, 2, and 3.

\begin{tabular}{|c|c|c|c|}
\hline Compounds & $\mathbf{1} \cdot 2\left(\mathrm{CH}_{2} \mathrm{Cl}_{2}\right)$ & 2 & $3 \cdot \mathrm{THF}$ \\
\hline Formula & $\mathrm{C}_{35} \mathrm{H}_{49} \mathrm{Cl}_{5} \mathrm{~N}_{4} \mathrm{O}_{3} \mathrm{Th}$ & $\mathrm{C}_{37} \mathrm{H}_{56} \mathrm{~N}_{4} \mathrm{O}_{3} \mathrm{SiTh}$ & $\mathrm{C}_{45} \mathrm{H}_{57} \mathrm{~F}_{6} \mathrm{~N}_{5} \mathrm{O}_{4} \mathrm{Th}$ \\
\hline$M_{\mathrm{r}}$ & 983.07 & 864.98 & 1077.99 \\
\hline$a[\AA]$ & $11.3484(7)$ & $9.7120(8)$ & $12.0934(15)$ \\
\hline$b[\AA]$ & $20.8006(12)$ & $17.5161(15)$ & $17.895(2)$ \\
\hline$c[\AA]$ & $17.0866(10)$ & $22.8464(17)$ & $20.998(3)$ \\
\hline$\alpha\left[^{\circ}\right]$ & 90 & 90 & 90 \\
\hline$\beta\left[^{\circ}\right]$ & $102.202(2)$ & $93.634(3)$ & $97.346(6)$ \\
\hline$\gamma\left[^{\circ}\right]$ & 90 & 90 & 90 \\
\hline$V\left[\AA^{3}\right]$ & $3942.2(4)$ & $3878.7(5)$ & $4506.8(10)$ \\
\hline$Z$ & 4 & 4 & 4 \\
\hline Space group & $P 2_{1} / c$ & $P 2_{1} / n$ & $P 2_{1} / n$ \\
\hline$\rho_{\text {calcd }},\left[\mathrm{g} \mathrm{cm}^{-3}\right]$ & 1.656 & 1.481 & 1.589 \\
\hline$\mu\left[\mathrm{mm}^{-1}\right]$ & 4.160 & 3.913 & 3.381 \\
\hline $2 \theta$ range $\left[{ }^{\circ}\right]$ & $5.792-55.114$ & $2.932-55.05$ & $3.00-55.008$ \\
\hline Data collected & 104793 & 91135 & 64753 \\
\hline No. unique data & 9076 & 8832 & 10324 \\
\hline$R_{\text {int }}$ & 0.0225 & 0.0449 & 0.0394 \\
\hline$R_{I}(\text { all data })^{[\mathrm{a}]}$ & 0.0186 & 0.0513 & 0.0228 \\
\hline$w R_{2}(\text { all data })^{[\mathrm{b}]}$ & 0.0479 & 0.1046 & 0.0547 \\
\hline $\mathrm{GoF}^{[\mathrm{c}]}$ & 1.251 & 1.157 & 1.067 \\
\hline No. of variables & 442 & 680 & 563 \\
\hline peak/hole $\left[\mathrm{e} \AA^{-3}\right.$ ] & $0.64 /-1.42$ & $3.86 /-2.96$ & $1.29 /-0.65$ \\
\hline $\mathrm{CCDC}$ & 1916859 & 1916860 & 1916861 \\
\hline
\end{tabular}

${ }^{[\mathrm{a}]} R_{1}=\Sigma|| F_{\mathrm{o}}|-| F_{\mathrm{c}}|| / 2\left|F_{\mathrm{o}}\right| \cdot{ }^{[\mathrm{b}]} w R_{2}=\left[\sum\left[w\left(F_{\mathrm{o}}{ }^{2}-F_{\mathrm{c}}{ }^{2}\right)^{2}\right] / 2\left[w\left(F_{\mathrm{o}}{ }^{2}\right)^{2}\right]\right]^{1 / 2} \cdot{ }^{[\mathrm{cc}]}$ Goodness-of-fit $\left[\sum\left[w\left(F_{\mathrm{o}}{ }^{2}-F_{\mathrm{c}}{ }^{2}\right)^{2}\right] /\left(N_{\mathrm{obs}}-N_{\mathrm{params}}\right)\right]^{1 / 2}$, based on all data. 
Table S2. Crystallographic data for $\mathbf{4}_{\mathrm{Li}}, \mathbf{4}_{\varnothing}$, and $\mathbf{6}_{\mathrm{Th}}$.

\begin{tabular}{|c|c|c|c|}
\hline Compounds & $4_{\mathrm{Li}}$ & $4_{\varnothing}$ & $\mathbf{6}_{\mathrm{Th}}$ \\
\hline Formula & $\mathrm{C}_{49} \mathrm{H}_{64} \mathrm{~F}_{6} \mathrm{LiN}_{5} \mathrm{O}_{5} \mathrm{Th}$ & $\mathrm{C}_{59} \mathrm{H}_{84} \mathrm{~F}_{6} \mathrm{KN}_{7} \mathrm{O}_{9} \mathrm{Th}$ & $\mathrm{C}_{69} \mathrm{H}_{67} \mathrm{BF}_{24} \mathrm{~N}_{4} \mathrm{O}_{4} \mathrm{Th}$ \\
\hline$M_{\mathrm{r}}$ & 1156.03 & 1420.47 & 1715.11 \\
\hline$a[\AA]$ & $13.9171(6)$ & $11.8126(6)$ & $19.4010(16)$ \\
\hline$b[\AA]$ & $18.1649(7)$ & $12.0421(6)$ & $19.4715(16)$ \\
\hline$c[\AA]$ & $22.1946(9)$ & $25.5193(12)$ & $18.6191(15)$ \\
\hline$\alpha\left[^{\circ}\right]$ & 90 & $94.897(2)$ & 90 \\
\hline$\beta\left[^{\circ}\right]$ & $96.724(2)$ & $95.005(2)$ & $97.543(4)$ \\
\hline$\gamma\left[^{\circ}\right]$ & 90 & $118.644(2)$ & 90 \\
\hline$V\left[\AA^{3}\right]$ & $5572.3(4)$ & $3139.9(3)$ & $6972.8(10)$ \\
\hline$Z$ & 4 & 2 & 4 \\
\hline Space group & $P 2_{1} / n$ & $P-1$ & $P 2_{1} / c$ \\
\hline$\rho_{\text {calcd }},\left[\mathrm{g} \mathrm{cm}^{-3}\right]$ & 1.378 & 1.502 & 1.634 \\
\hline$\mu\left[\mathrm{mm}^{-1}\right]$ & 2.740 & 2.517 & 2.254 \\
\hline $2 \theta$ range $\left[{ }^{\circ}\right]$ & $5.788-55.104$ & $6.264-55.112$ & $2.976-55.074$ \\
\hline Data collected & 250399 & 103603 & 172244 \\
\hline No. unique data & 12827 & 14451 & 16040 \\
\hline$R_{\text {int }}$ & 0.0424 & 0.0748 & 0.0390 \\
\hline$R_{l}$ (all data) ${ }^{[\mathrm{a}]}$ & 0.0539 & 0.0564 & 0.0450 \\
\hline$w R_{2}(\text { all data })^{[\mathrm{b}]}$ & 0.1463 & 0.1059 & 0.0892 \\
\hline $\mathrm{GoF}^{[c]}$ & 1.293 & 1.161 & 1.025 \\
\hline No. of variables & 703 & 757 & 1493 \\
\hline peak/hole $\left[\mathrm{e} . \AA^{-3}\right]$ & $3.65 /-4.30$ & $2.763 /-3.27$ & $4.44 /-3.69$ \\
\hline CCDC & 1916862 & 1916863 & 1916864 \\
\hline
\end{tabular}




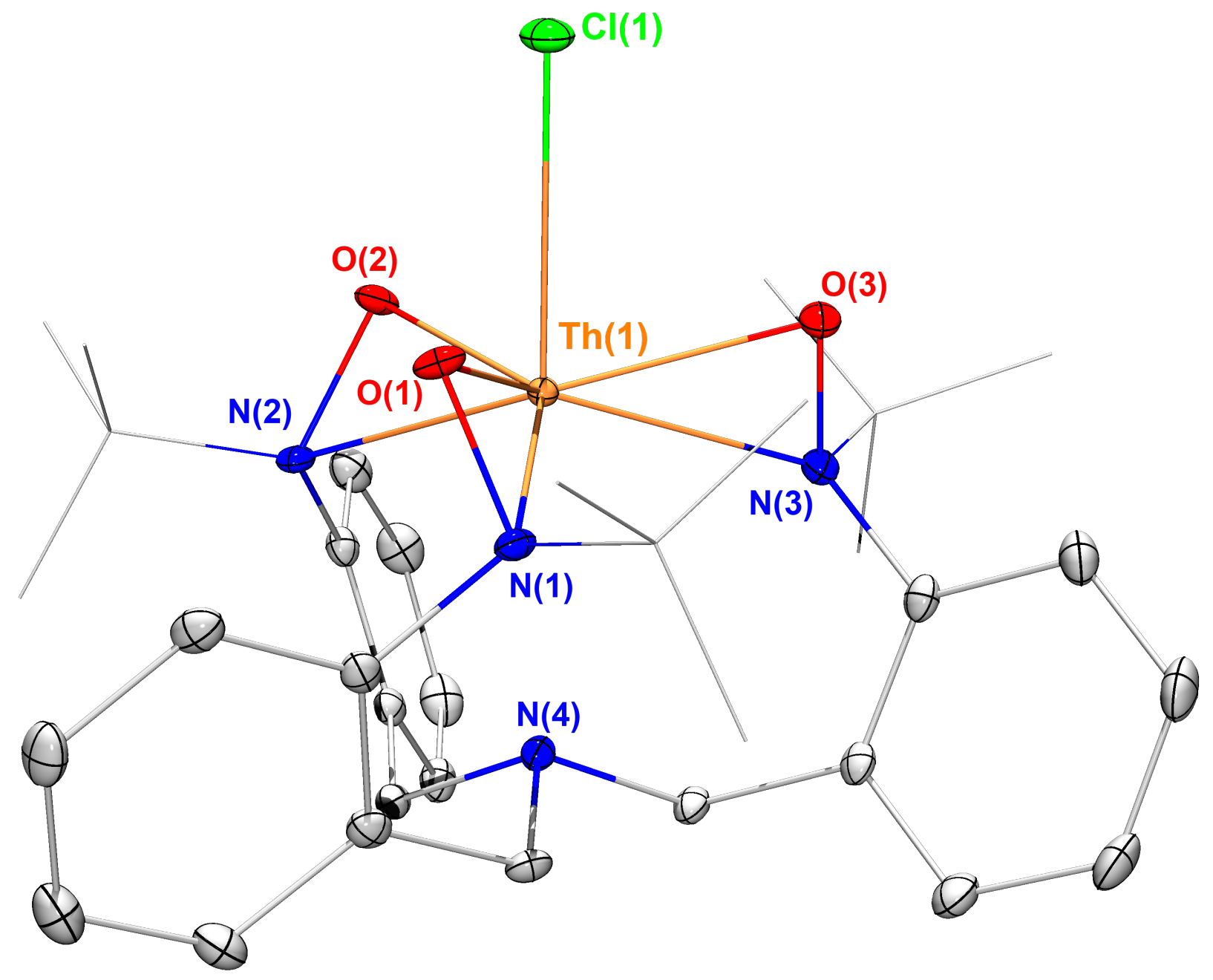

Figure S17. Thermal ellipsoid plot (50\% probability) of $\mathbf{1} \cdot 2\left(\mathrm{CH}_{2} \mathrm{Cl}_{2}\right)$. Hydrogen atoms and two co-crystallized dichloromethane molecules are omitted, tert-butyl groups are depicted in a wireframe model for clarity. Selected bond lengths $(\AA)$ and angles $\left(^{\circ}\right)$ : $\operatorname{Th}(1)-\mathrm{Cl}(1) 2.7536(7)$, $\mathrm{Th}(1)-\mathrm{N}(4)$ 2.876(2), Th(1)-O(1) 2.248(2), Th(1)-O(2) 2.252(2), Th(1)-O(3) 2.256(2), Th(1)$\mathrm{N}(1) 2.576(2), \mathrm{Th}(1)-\mathrm{N}(2) 2.574(2), \mathrm{Th}(1)-\mathrm{N}(3)$ 2.567(2); N(4)-Th(1)-Cl(1) 179.81(4). 


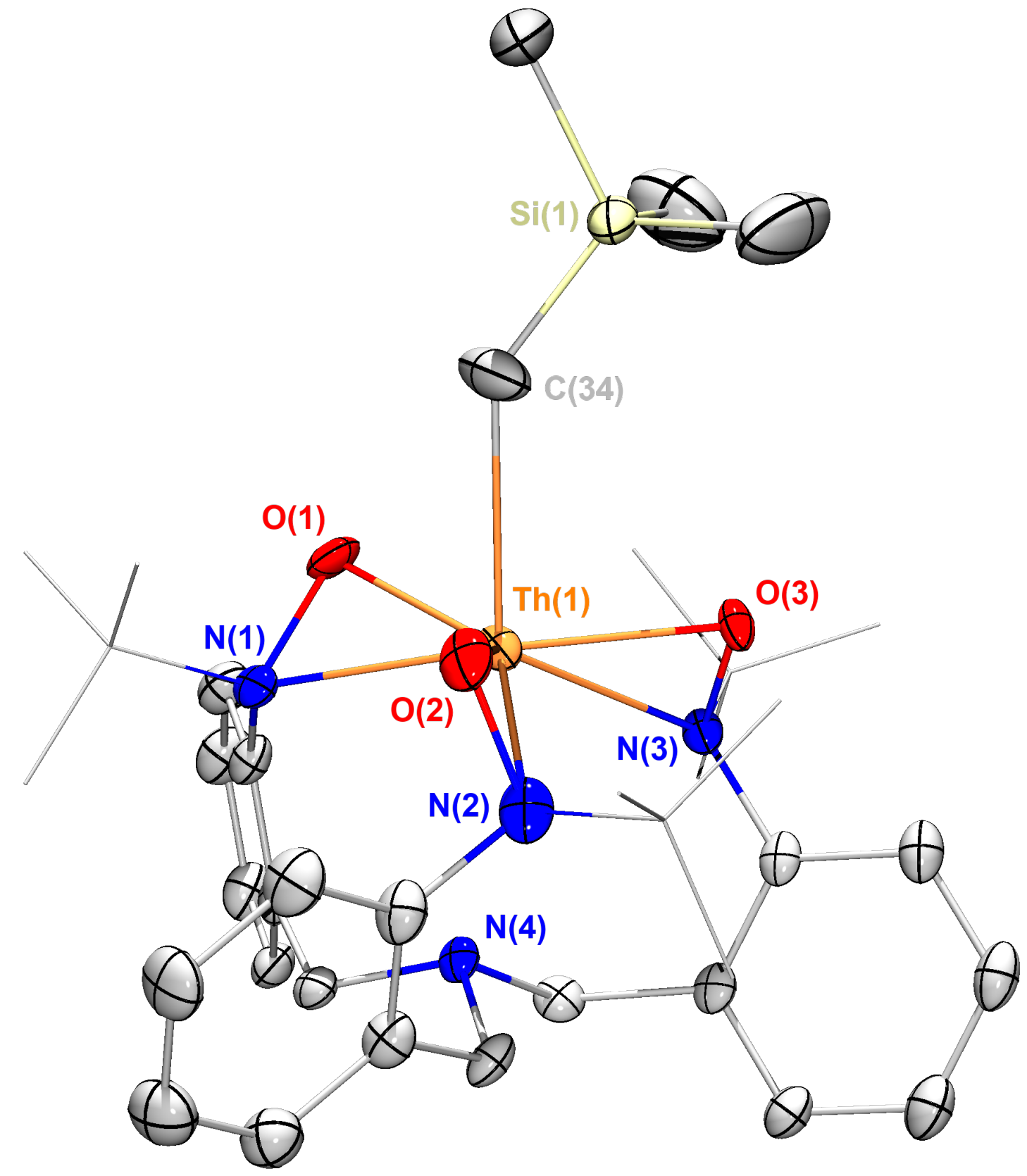

Figure S18. Thermal ellipsoid plot (50\% probability) of 2. Hydrogen atoms are omitted, tertbutyl groups are depicted in a wireframe model for clarity. Selected bond lengths $(\AA)$ and angles $\left({ }^{\circ}\right): \operatorname{Th}(1)-\mathrm{C}(34)$ 2.568(7), Th(1)-N(4) 3.020(5), Th(1)-O(1) 2.216(9), Th(1)-O(2) 2.265(9), $\mathrm{Th}(1)-\mathrm{O}(3)$ 2.308(9), Th(1)-N(1) 2.55(1), Th(1)-N(2) 2.62(1), Th(1)-N(3) 2.626(9); N(4)$\mathrm{Th}(1)-\mathrm{C}(34)$ 171.9(2), Th(1)-C(34)-Si(1) 139.1(4). 


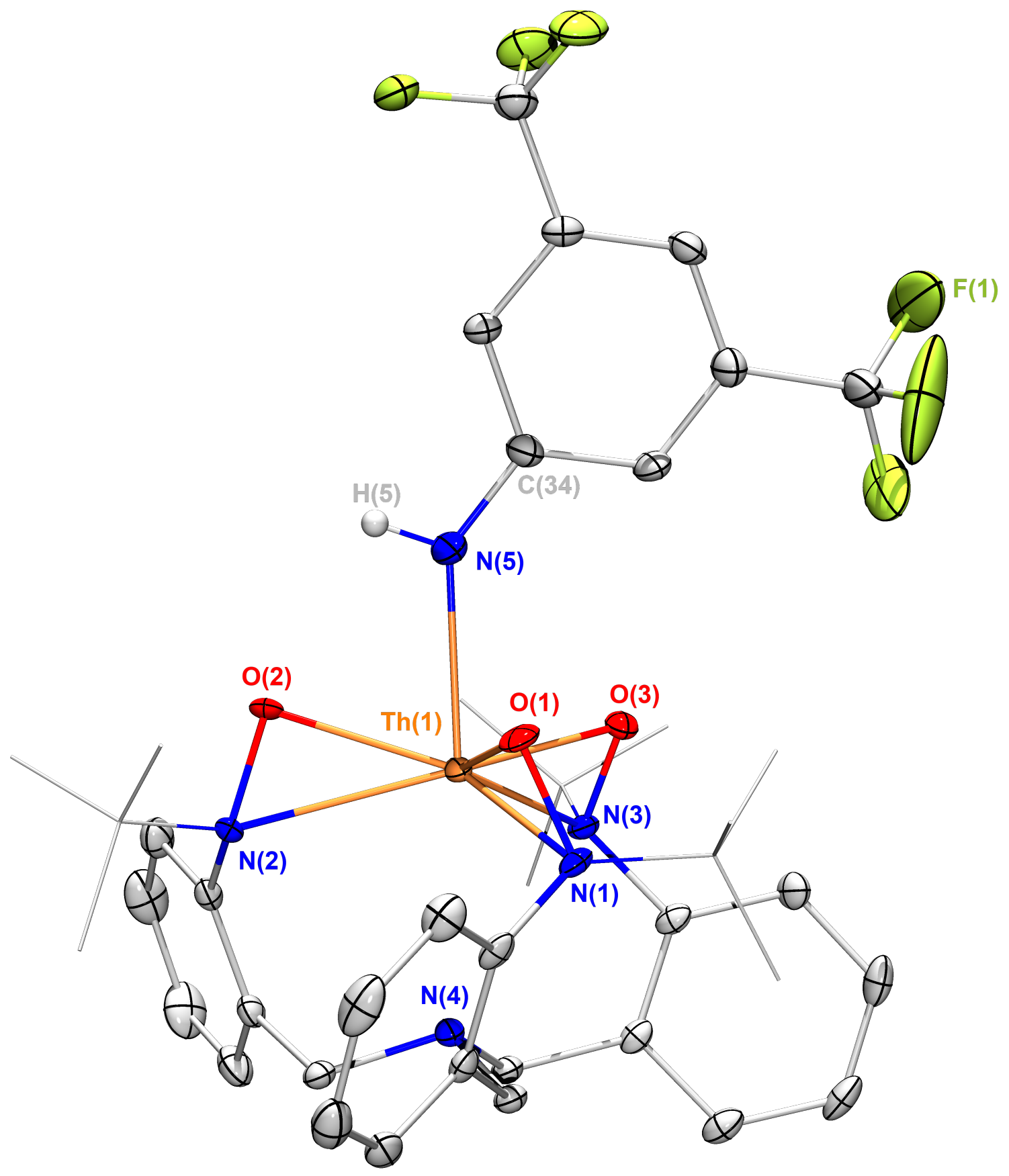

Figure S19. Thermal ellipsoid plot (50\% probability) of 3.THF. Most hydrogen atoms and a cocrystallized molecule are omitted, tert-butyl groups are depicted in a wireframe model for clarity. $\mathrm{H}(5)$ was localized on the electron density map and refined isotropically in position. Selected bond lengths $(\AA)$ and angles $\left(^{\circ}\right)$ : Th(1)-N(5) 2.430(3), N(5)-H(5) 0.71(4), Th(1)-N(4) 2.941(2), $\mathrm{Th}(1)-\mathrm{O}(1)$ 2.265(2), Th(1)-O(2) 2.233(2), Th(1)-O(3) 2.275(2), Th(1)-N(1) 2.571(2), Th(1)$\mathrm{N}(2)$ 2.570(2), Th(1)-N(3) 2.605(3); Th(1)-N(5)-C(34) 142.5(2), N(4)-Th(1)-N(5) 174.51(7), $\mathrm{H}(5)-\mathrm{N}(5)-\mathrm{C}(34) 108(3)$. 

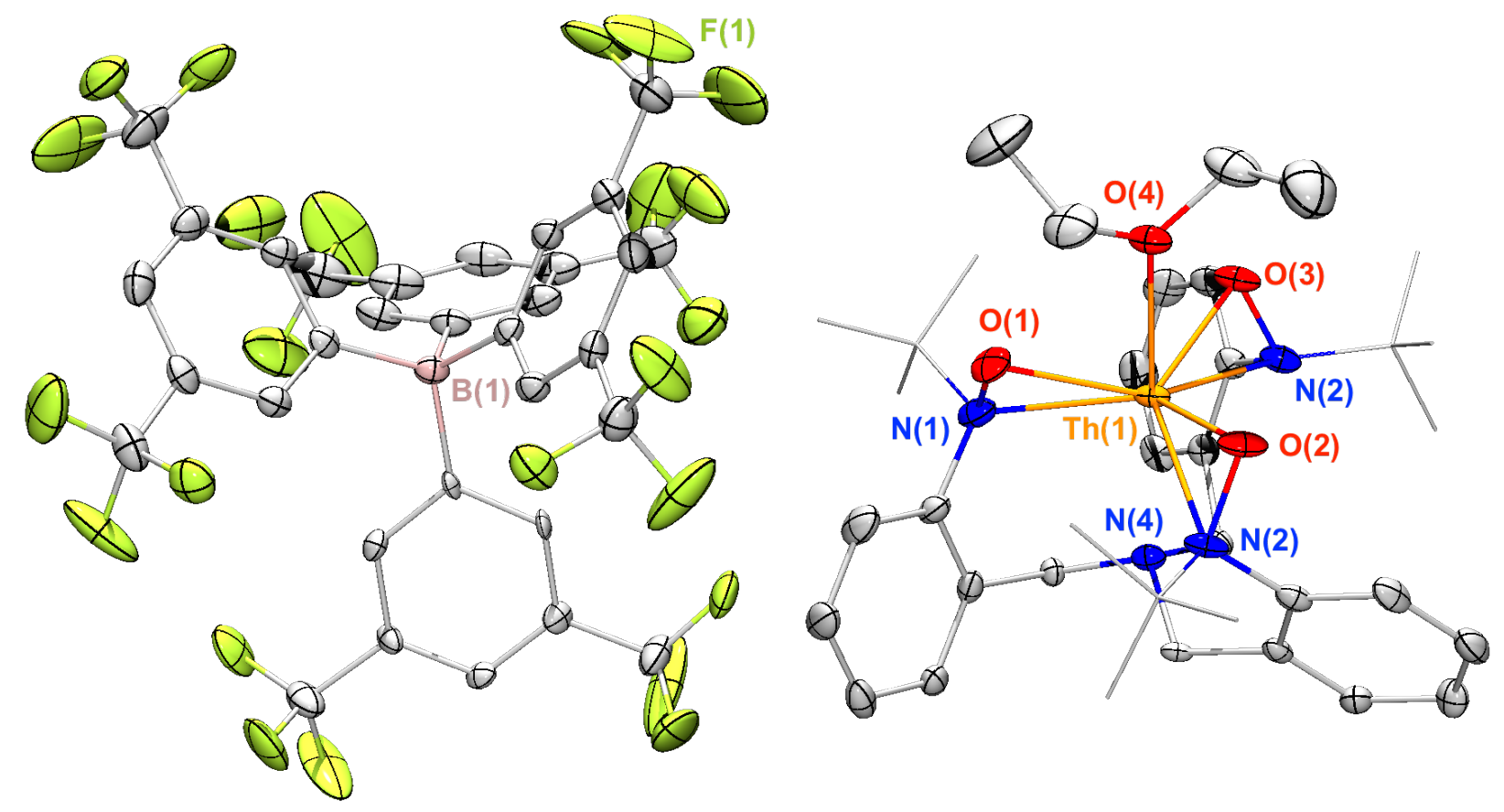

Figure S20. Thermal ellipsoid plot (50\% probability) of $\mathbf{6}_{\mathbf{T h}}$. Hydrogen atoms are omitted, tertbutyl groups are depicted in a wireframe model for clarity. Selected bond lengths $(\AA)$ and angles $\left(^{\circ}\right): \mathrm{Th}(1)-\mathrm{O}(4)$ 2.562(4), $\mathrm{Th}(1)-\mathrm{N}(4)$ 2.795(3), Th(1)-O(1) 2.234(4), $\mathrm{Th}(1)-\mathrm{O}(2)$ 2.261(4), $\mathrm{Th}(1)-\mathrm{O}(3)$ 2.228(4), Th(1)-N(1) 2.514(6), Th(1)-N(2) 2.555(4), Th(1)-N(3) 2.553(5); N(4)$\mathrm{Th}(1)-\mathrm{O}(4) 178.5(1)$. 
Electronic Absorption Spectroscopy. UV-Visible spectra were obtained using a Perkin Elmer $950 \mathrm{UV}-\mathrm{Vis} / \mathrm{NIR}$ Spectrophotometer. Solutions of known concentration were prepared under $\mathrm{N}_{2}$ atmosphere and transferred to a, airtight, screw cap, quartz cell of $1 \mathrm{~cm}$ path-length. Blanks were measured prior to each run.

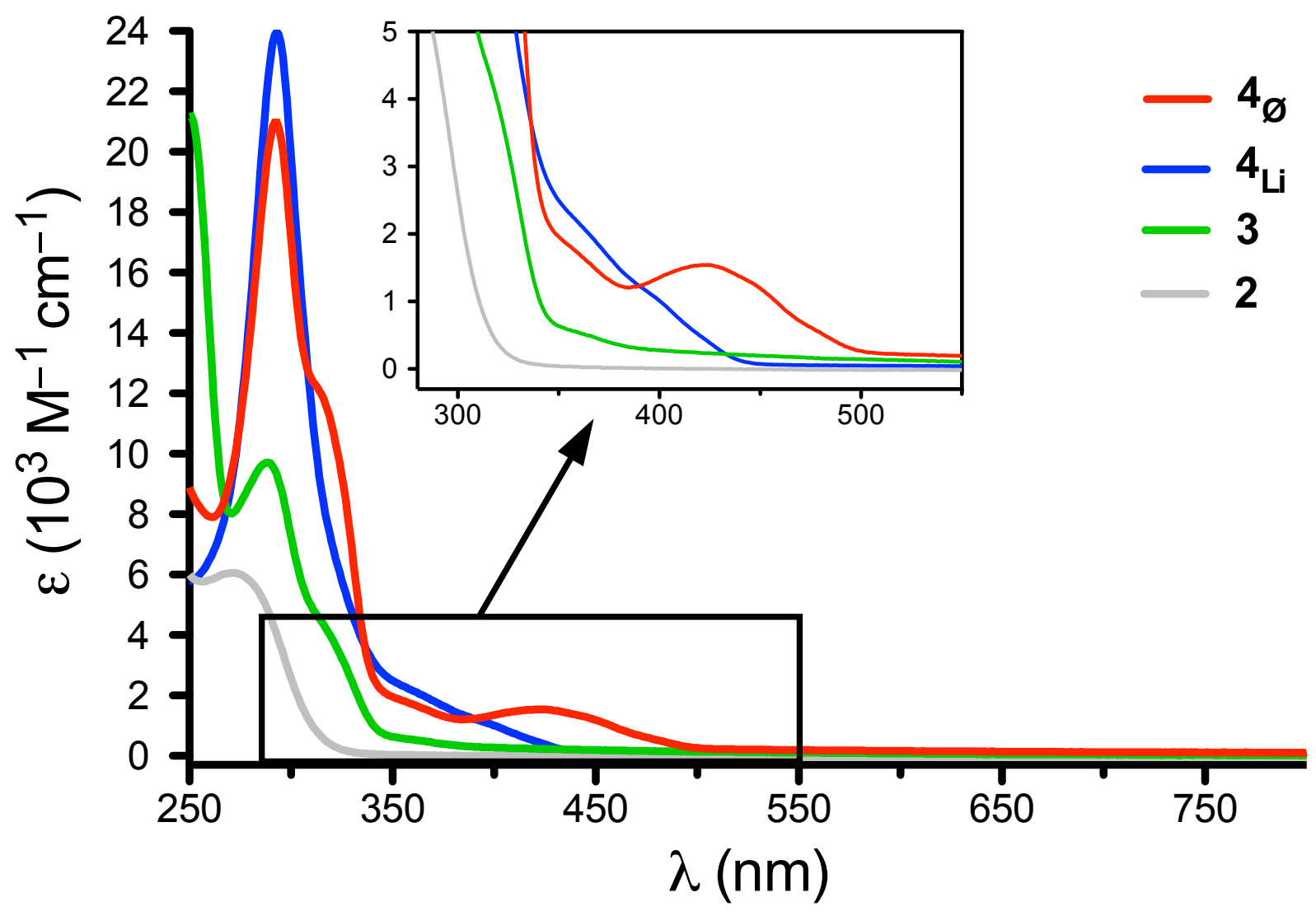

Figure S21. UV-Visible spectra of $\mathbf{2}, \mathbf{3}, \mathbf{4}_{\mathrm{Li}}$, and $\mathbf{4}_{\varnothing}$ collected in THF at $300 \mathrm{~K}$. 
Infrared Spectroscopy. $\mathrm{KBr}$ pellets of $\mathbf{3}$ and $\mathbf{4}_{\mathrm{Li}}$ were prepared in a nitrogen-filled drybox and transferred in the air to a Perkin Elmer 1600 series infrared spectrometer. The infrared spectra were obtained from $400-4000 \mathrm{~cm}^{-1}$.

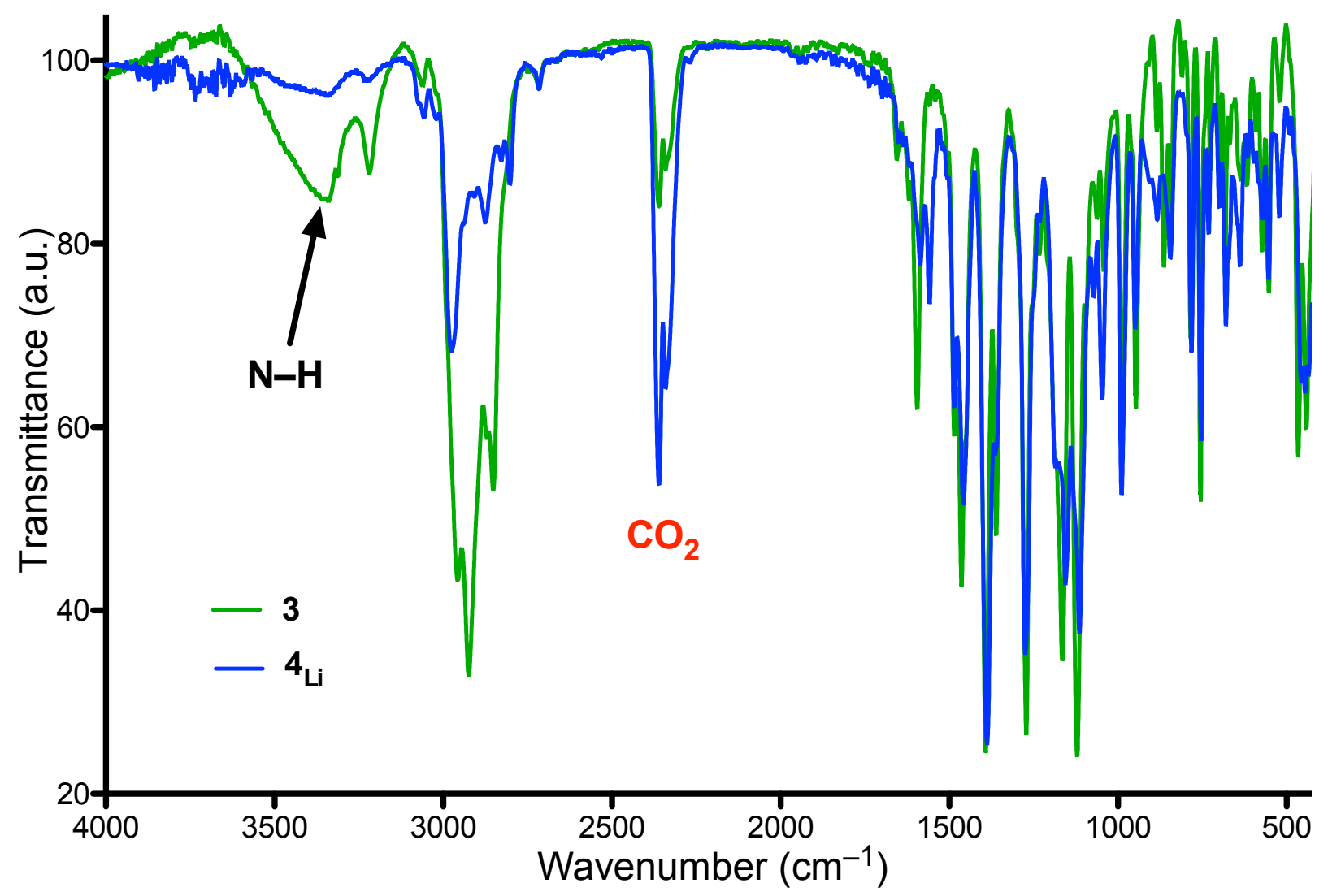

Figure S22. Infrared spectra of $\mathbf{2}$ and $\mathbf{4}_{\mathrm{Li}}$ (KBr pellets) collected at room temperature. 
Electrochemistry. Cyclic voltammetry experiments were performed using a $\mathrm{CH}$ Instruments 620D Electrochemical Analyzer/Workstation and the data were processed using CHI software v9.24. All experiments were performed in an $\mathrm{N}_{2}$ atmosphere drybox using electrochemical cells that consisted of a $4 \mathrm{~mL}$ vial, glassy carbon working electrode, a platinum wire counter electrode, and a silver wire plated with $\mathrm{AgCl}$ as a quasi-reference electrode. The quasi-reference electrode was prepared by dipping a length of silver wire in concentrated hydrochloric acid. The working electrode surfaces were polished prior to each set of experiments. Potentials were reported versus ferrocene, which was added as an internal standard for calibration at the end of each run. Solutions employed were $\sim 3 \mathrm{mM}$ in analyte and $100 \mathrm{mM}$ in $\left[{ }^{n} \operatorname{Pr}_{4} \mathrm{~N}\right]\left[\mathrm{BAr}^{\mathrm{F}}{ }_{4}\right]$ in $2 \mathrm{~mL}$ of DME. All data were collected in a positive-feedback IR compensation mode. The rapid decomposition of $\mathbf{4}_{\varnothing}$ under electrochemical conditions prevented the collection of meaningful data for this compound.

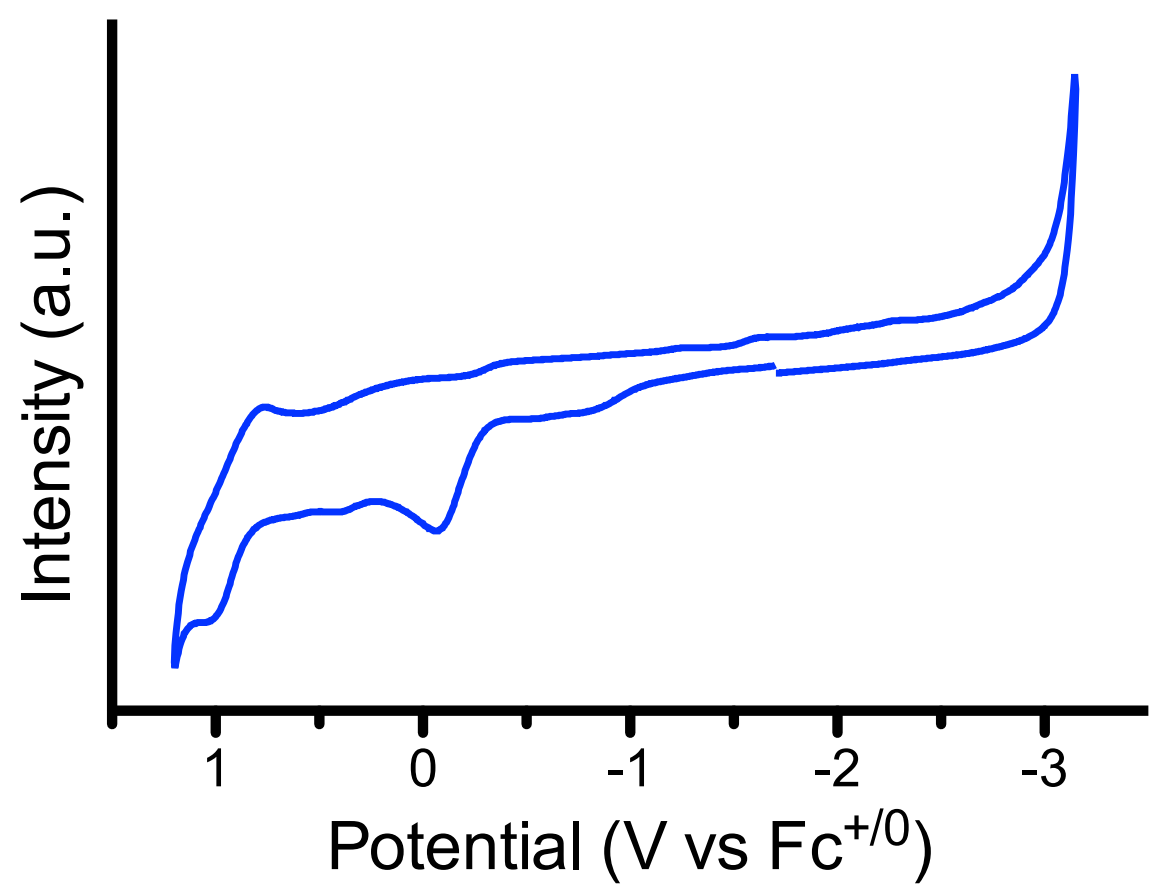

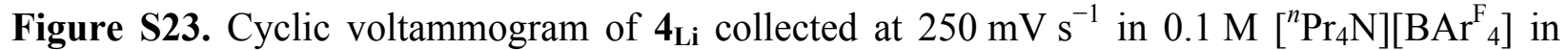
DME. The oxidation electrochemical events observed in the oxidation are attributed to the redox activity of $\mathrm{TriNOx}^{3-}$ as observed in related systems. ${ }^{13}$ 
Acidity Constants Determination. All bracketing experiments were performed according to the following procedure: in a vial, the anilide complexes $\mathbf{3}$ or $\mathbf{3}_{\mathrm{Ce}}(\sim 10 \mathrm{mg}, 1$ equiv) was dissolved in THF- $d_{8}(\sim 0.4 \mathrm{~mL})$. In a separate vial, the selected base (1.1 equiv) was dissolved in THF- $d_{8}$ $(\sim 0.2 \mathrm{~mL})$. The two solutions were mixed and transferred to a J-Young NMR tube for spectroscopic analysis.

$\left[\operatorname{Th}\left(\mathrm{NHAr}^{\mathrm{F}}\right)(\operatorname{TriNOx})\right](3)+\mathrm{LiN}\left({ }^{\mathrm{P} P}\right)_{2} \mathrm{p} K_{\mathrm{a}}=35.6$

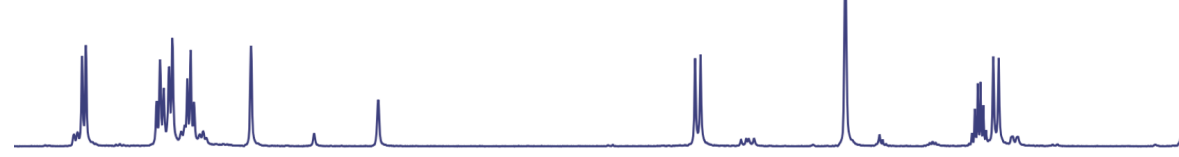

$\left[\operatorname{Th}\left(\mathrm{NHAr}^{\mathrm{F}}\right)(\operatorname{TriNOx})\right](3)+\mathrm{LiN}\left(\mathrm{SiMe}_{3}\right)\left({ }^{\mathrm{t}} \mathrm{Bu}\right) \mathrm{p} K_{\mathrm{a}}=33.6$

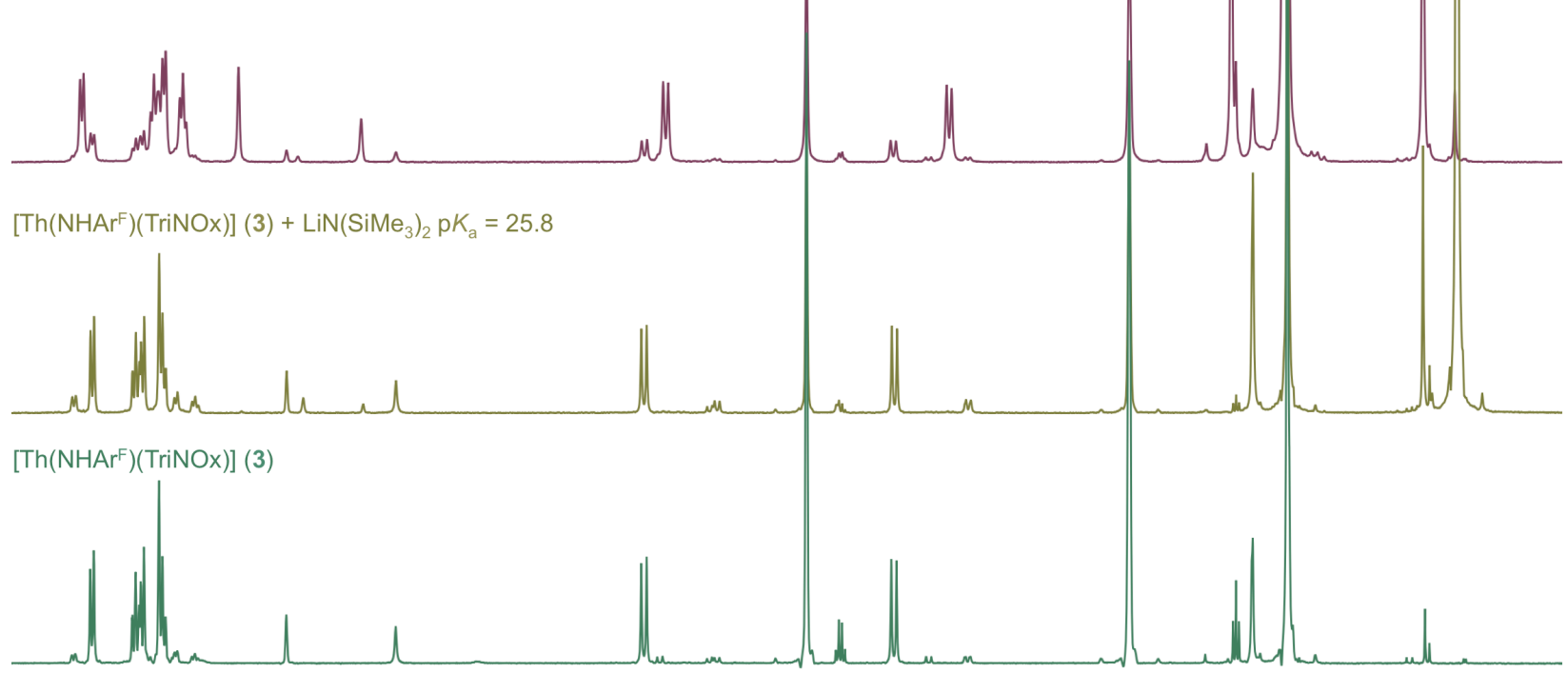

8.0

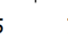

6.5

Figure S24. Overlay of the ${ }^{1} \mathrm{H}$ NMR spectra recorded at $300 \mathrm{~K}\left(\mathrm{THF}-d_{8},{ }^{*}\right)$ of $\mathbf{3}, \mathbf{3}+$ $\mathrm{LiN}\left(\mathrm{SiMe}_{3}\right)_{2}, \mathbf{3}+\mathrm{LiN}\left(\mathrm{SiMe}_{3}\right)\left({ }^{t} \mathrm{Bu}\right)$, and $\mathbf{3}+\mathrm{LiN}\left({ }^{i} \mathrm{Pr}\right)_{2}$. This bracketing allowed narrowing of the $\mathrm{p} K_{\mathrm{a}}$ of 3 to the $27-33$ range.

Accurate $\mathrm{p} K_{\mathrm{a}}$ determination of $\mathbf{3}$ was performed by ${ }^{1} \mathrm{H}$ NMR titration of $\mathbf{4}_{\mathbf{L i}}(10 \mathrm{mg})$ in THF- $d_{8}$ $(0.4 \mathrm{~mL})$ by a THF- $d_{8}$ solution of $\mathrm{HN}\left(\mathrm{SiMe}_{3}\right)\left({ }^{i} \mathrm{Pr}\right)(53 \mathrm{mM})$ using 1,3,5-trimethoxybenzene as an internal standard (IS). ${ }^{1} \mathrm{H}$ NMR spectra were acquired immediately and $30 \mathrm{~min}$ after each addition to confirm proper equilibration. The experimental spectra are depicted on Figure S25 and the results are summarized in Table S3. 

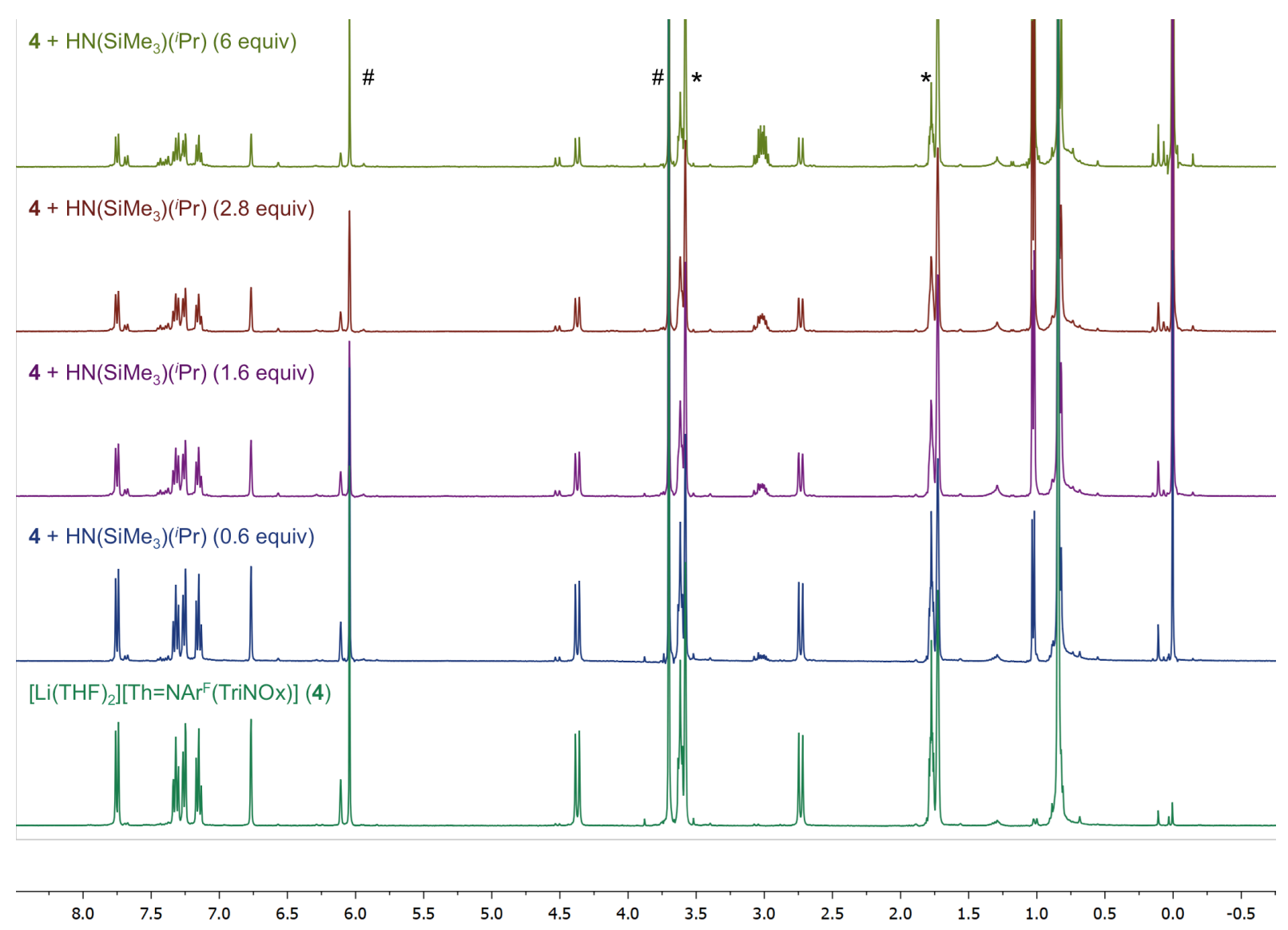

Figure S25. ${ }^{1} \mathrm{H}$ NMR titration of $\mathbf{4}_{\mathbf{L i}}$ with incremental amounts of $\mathrm{HN}\left(\mathrm{SiMe}_{3}\right)\left({ }^{i} \mathrm{Pr}\right)$ in $\mathrm{THF}-d_{8}$ using 1,3,5-trimethoxybenzene (\#) as an internal standard.

The equilibrium constant $K_{\text {Th }}$ was calculated for each titration points by ${ }^{1} \mathrm{H}$ NMR integrations of characteristic resonances $K_{\mathrm{Th}}=\frac{\left[\mathbf{4}_{\mathrm{Li}}\right]\left[\mathrm{HN}\left(\mathrm{SiMe}_{3}\right)\left({ }^{i} \mathrm{Pr}\right)\right]}{[\mathbf{3}]\left[\mathrm{LiN}\left(\mathrm{SiMe}_{3}\right)\left({ }^{i} \mathrm{Pr}\right)\right]}$ of $\mathbf{4}_{\mathbf{L i}}, \mathbf{3}, \mathrm{LiN}\left(\mathrm{SiMe}_{3}\right)\left({ }^{i} \mathrm{Pr}\right)$, and $\mathrm{HN}\left(\mathrm{SiMe}_{3}\right)\left({ }^{i} \mathrm{Pr}\right)$ against the IS. $\mathrm{p} K_{\mathrm{a}}(\mathbf{3})$ was determined according to the equation: $\mathrm{p} K_{\mathrm{a}}(\mathbf{3})=\mathrm{p} K_{\mathrm{a}}\left(\mathrm{HN}\left(\mathrm{SiMe}_{3}\right)\left({ }^{i} \mathrm{Pr}\right)\right)-\log _{10}\left(K_{\mathrm{Th}}\right)$. Accounting for $5 \%$ errors on the ${ }^{1} \mathrm{H}$ NMR integration values, the titration gave $\mathrm{p} K_{\mathrm{a}}(3)=28.6(3)$.

Table S3. ${ }^{1} \mathrm{H}$ NMR integrations for the titration of $\mathbf{4}_{\mathbf{L i}}$ with $\mathrm{HN}\left(\mathrm{SiMe}_{3}\right)\left({ }^{i} \mathrm{Pr}\right)$, and the associated equilibrium constants $K_{\mathrm{Th}}$ and the resulting $\mathrm{p} K_{\mathrm{a}}$ values for $\mathbf{3}$.

\begin{tabular}{cccccc|cc}
$\begin{array}{c}\mathrm{HN}\left(\mathrm{SiMe}_{3}\right)\left({ }^{i} \mathrm{Pr}\right) \\
\text { equiv }\end{array}$ & $\mathrm{IS}$ & $\mathbf{3}$ & $\mathbf{4}_{\mathbf{L i}}$ & $\mathrm{LiN}\left(\mathrm{SiMe}_{3}\right)\left({ }^{i} \mathrm{Pr}\right)$ & $\mathrm{HN}\left(\mathrm{SiMe}_{3}\right)\left({ }^{i} \mathrm{Pr}\right)$ & $\begin{array}{c}K_{\mathrm{Th}} \\
\left(\times 10^{2}\right)\end{array}$ & $\mathrm{p} K_{\mathrm{a}}$ \\
\hline 0.6 & 100 & 0.06 & 2.05 & 0.04 & 3.70 & 31.6 & 28.10 \\
1.6 & 100 & 0.23 & 1.86 & 0.09 & 10.86 & 9.62 & 28.61 \\
2.8 & 100 & 0.29 & 1.80 & 0.15 & 18.56 & 7.63 & 28.71 \\
6 & 100 & 0.48 & 1.53 & 0.27 & 40.12 & 4.72 & 28.92
\end{tabular}

$\mathrm{p} K_{\mathrm{a}}(\mathbf{3})$ was also determined by titration of $\mathbf{3}$ with $\mathrm{LiN}\left(\mathrm{SiMe}_{3}\right)_{2}$ and returned a similar value of 28.3(3) but due to the limited quality of this dataset, the previous titration was preferred. 

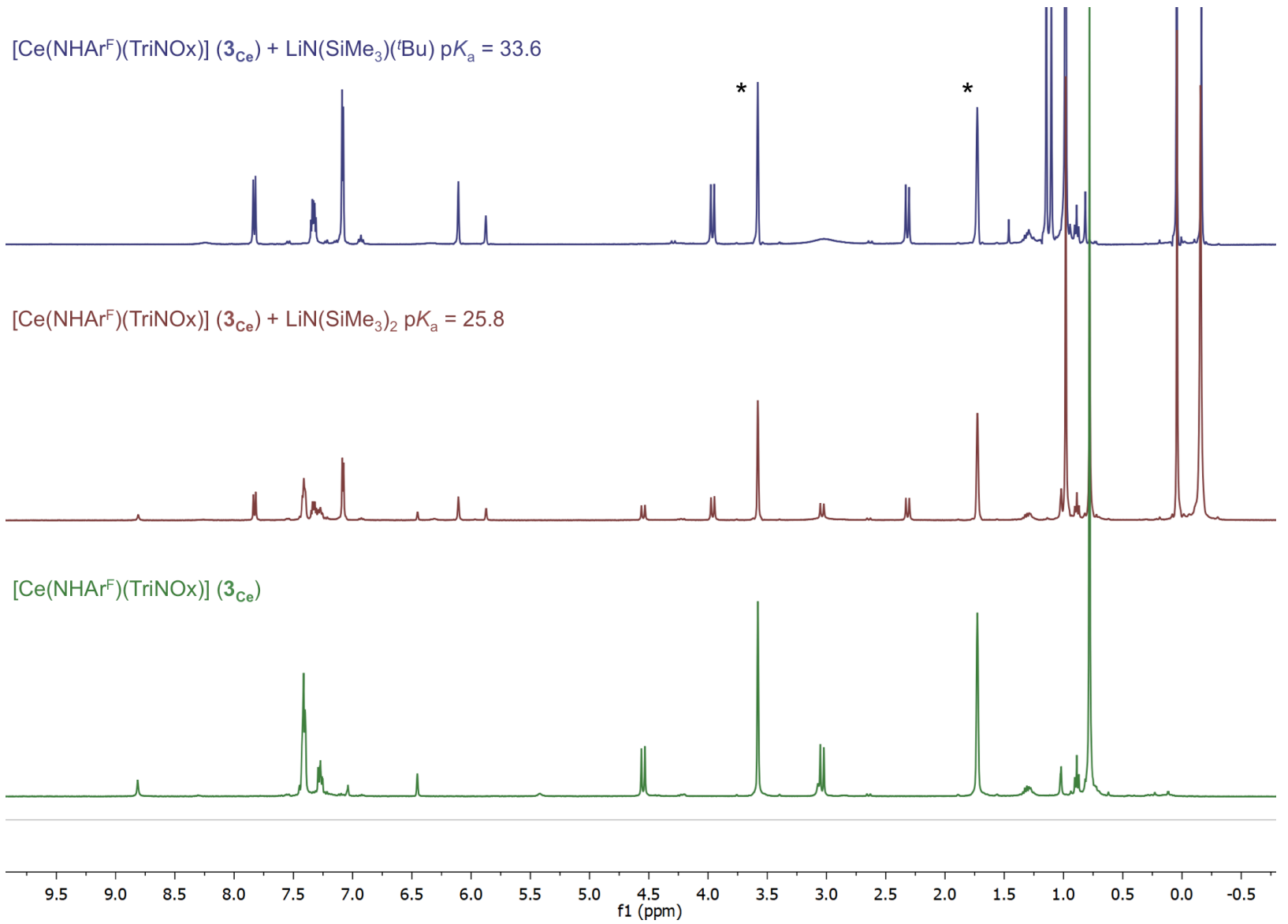

Figure S26. Overlay of the ${ }^{1} \mathrm{H}$ NMR spectra recorded at $300 \mathrm{~K}\left(\mathrm{THF}-d_{8},{ }^{*}\right)$ of $\mathbf{3}_{\mathrm{Ce}}, \mathbf{3}_{\mathrm{Ce}}+$ $\mathrm{LiN}\left(\mathrm{SiMe}_{3}\right)_{2}$, and $\mathbf{3}_{\mathrm{Ce}}+\mathrm{LiN}\left(\mathrm{SiMe}_{3}\right)\left({ }^{t} \mathrm{Bu}\right)$. This bracketing allowed narrowing of the $\mathrm{p} K_{\mathrm{a}}$ of $\mathbf{3}_{\mathrm{Ce}}$ to the 22-28 range.

Accurate $\mathrm{p} K_{\mathrm{a}}$ determination of $\mathbf{3}_{\mathrm{Ce}}$ was performed by ${ }^{1} \mathrm{H}$ NMR titration of $\mathbf{3}_{\mathrm{Ce}}(10 \mathrm{mg})$ in THF- $d_{8}$ $(0.4 \mathrm{~mL})$ by a THF- $d_{8}$ solution of $\mathrm{LiN}\left(\mathrm{SiMe}_{3}\right)_{2}(11 \mathrm{mM})$ using 1,3,5-trimethoxybenzene as an internal standard (IS). ${ }^{1} \mathrm{H}$ NMR spectra were acquired immediately and $30 \mathrm{~min}$ after each addition to confirm proper equilibration. The experimental spectra are depicted on Figure S27 and the results are summarized in Table S4. 

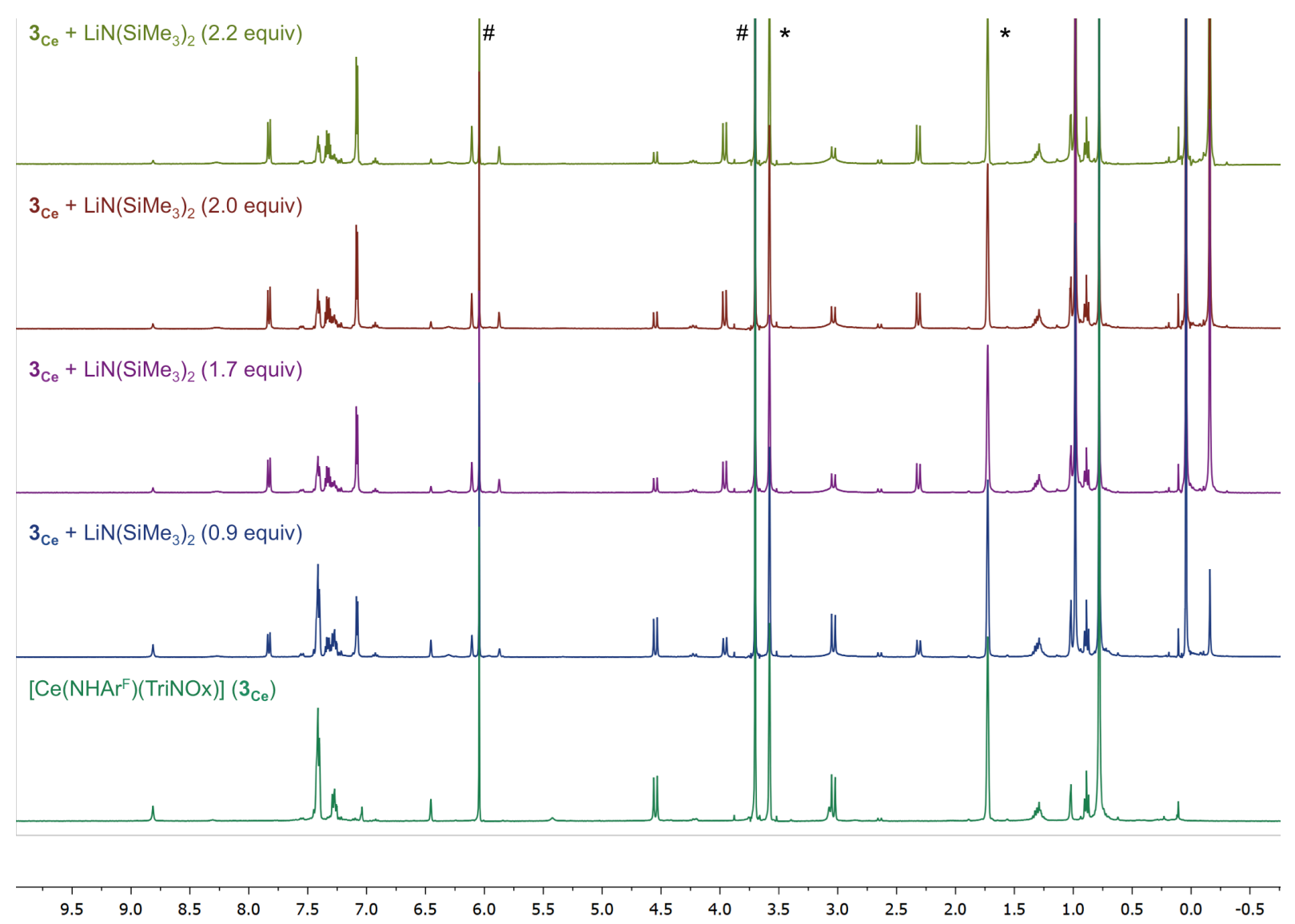

Figure S27. ${ }^{1} \mathrm{H}$ NMR titration of $\mathbf{3}_{\mathrm{Ce}}$ with incremental amounts of $\mathrm{LiN}\left(\mathrm{SiMe}_{3}\right)_{2}$ in $\mathrm{THF}-d_{8}(*)$ using 1,3,5-trimethoxybenzene (\#) as an internal standard.

The equilibrium constant $K_{\mathrm{Ce}}$ was calculated for each titration points by ${ }^{1} \mathrm{H}$ NMR integrations of characteristic resonances of $\quad K_{\mathrm{Ce}}=\frac{\left[\mathbf{5}_{\mathbf{L i}}\right]\left[\mathrm{HN}\left(\mathrm{SiMe}_{3}\right)_{2}\right]}{\left[\mathbf{3}_{\mathrm{Ce}}\right]\left[\mathrm{LiN}\left(\mathrm{SiMe}_{3}\right)_{2}\right]}$ $\mathbf{3}_{\mathbf{C e}}, \mathbf{5}_{\mathbf{L i}}, \quad \mathrm{LiN}\left(\mathrm{SiMe}_{3}\right)_{2}$, and $\mathrm{HN}\left(\mathrm{SiMe}_{3}\right)_{2}$ against the IS.

$$
K_{\mathrm{Ce}}=\frac{\left[\mathbf{5}_{\mathbf{L i}}\right]\left[\mathrm{HN}\left(\mathrm{SiMe}_{3}\right)_{2}\right]}{\left[\mathbf{3}_{\mathrm{Ce}}\right]\left[\mathrm{LiN}\left(\mathrm{SiMe}_{3}\right)_{2}\right]}
$$
$\mathrm{p} K_{\mathrm{a}}\left(\mathbf{3}_{\mathrm{Ce}}\right)$ was determined according to the equation: $\mathrm{p} K_{\mathrm{a}}\left(\mathbf{3}_{\mathrm{Ce}}\right)=\mathrm{p} K_{\mathrm{a}}\left(\mathrm{HN}\left(\mathrm{SiMe}_{3}\right)_{2}\right)-\log _{10}\left(K_{\mathrm{Ce}}\right)$. Accounting for $5 \%$ errors on the ${ }^{1} \mathrm{H}$ NMR integration values, the titration gave $\mathrm{p} K_{\mathrm{a}}\left(\mathbf{3}_{\mathrm{Ce}}\right)=$ 25.2(2).

Table S4. ${ }^{1} \mathrm{H}$ NMR integrations for the titration of $\mathbf{3}_{\mathrm{Ce}}$ by $\mathrm{LiN}\left(\mathrm{SiMe}_{3}\right)_{2}$, and the associated equilibrium constants $K_{\mathrm{Ce}}$ and the resulting $\mathrm{p} K_{\mathrm{a}}$ values for $\mathbf{3}_{\mathrm{Ce}}$.

\begin{tabular}{cccccc|cc}
$\mathrm{LiN}\left(\mathrm{SiMe}_{3}\right)_{2}$ equiv & $\mathrm{IS}$ & $\mathbf{3}_{\mathbf{C e}}$ & $\mathbf{5}_{\mathbf{L i}}$ & $\mathrm{LiN}\left(\mathrm{SiMe}_{3}\right)_{2}$ & $\mathrm{HN}\left(\mathrm{SiMe}_{3}\right)_{2}$ & $K_{\mathrm{Ce}}$ & $\mathrm{p} K_{\mathrm{a}}$ \\
\hline 0 & 9 & 1.63 & 0 & $/$ & $/$ & $/$ & $/$ \\
0.9 & 9 & 1.34 & 0.93 & 1.76 & 10.36 & 4.09 & 25.19 \\
1.7 & 9 & 0.75 & 1.75 & 10.25 & 15.72 & 3.58 & 25.25 \\
2.0 & 9 & 0.66 & 1.74 & 11.20 & 17.35 & 4.08 & 25.19 \\
2.2 & 9 & 0.64 & 2.21 & 18.55 & 19.75 & 3.68 & 25.23
\end{tabular}



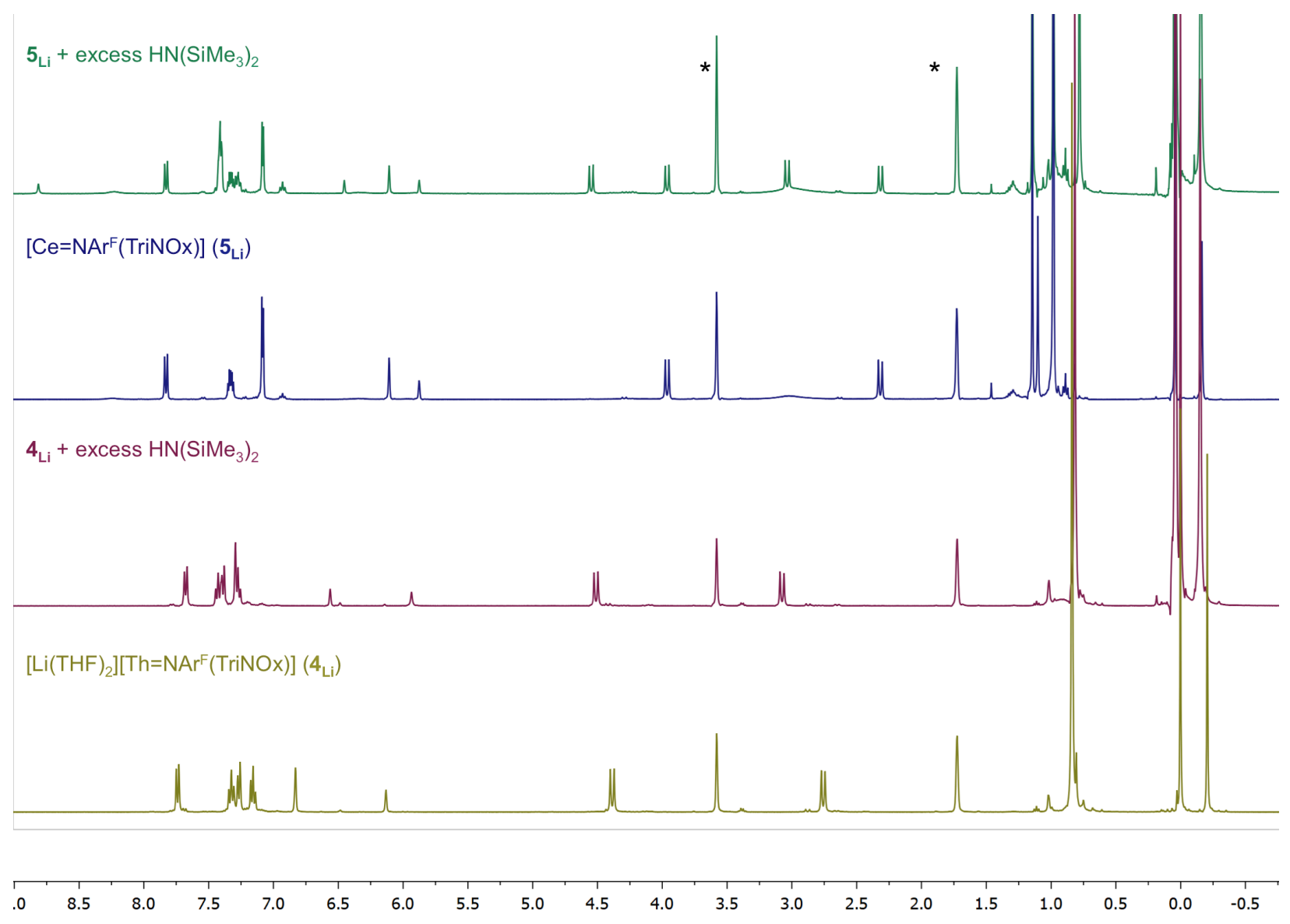

Figure S28. Re-protonation experiments of $\mathbf{4}_{\mathbf{L i}}$ and $\mathbf{5}_{\mathbf{L i}}$ in THF- $d_{8}\left(^{*}\right)$ with an excess of $\mathrm{HN}\left(\mathrm{SiMe}_{3}\right)_{2}$. 
Reactivity Studies. All experiments were performed according to the following protocol: in a vial, $\mathbf{4}_{\mathrm{Li}}$ or $\mathbf{4}_{\varnothing}\left(7-10 \mathrm{mg}, 1\right.$ equiv) were suspended in $\sim 0.4 \mathrm{~mL}$ of THF- $d_{8}$ or toluene- $d_{8}$. In a separate vial, $\mathbf{6}_{\mathrm{Ce}}\left(8-12 \mathrm{mg}, 1\right.$ equiv) was dissolved in $\sim 0.4 \mathrm{~mL}$ of THF- $d_{8}$ or toluene- $d_{8}$. The two solutions were quickly mixed leading to a rapid homogenization of the mixture to yield a lightbrown solution, which was transferred to a J-Young NMR tube for NMR analysis. After about 4 hours, a small amount of yellow precipitate identified as [Ce(THF)(TriNOx)] (7) was observed when the reaction was performed in THF- $d_{8}$.

- Reaction of $\mathbf{4}_{\varnothing}$ and $\mathbf{6}_{\mathrm{Ce}}$ in THF- $d_{8}$ :
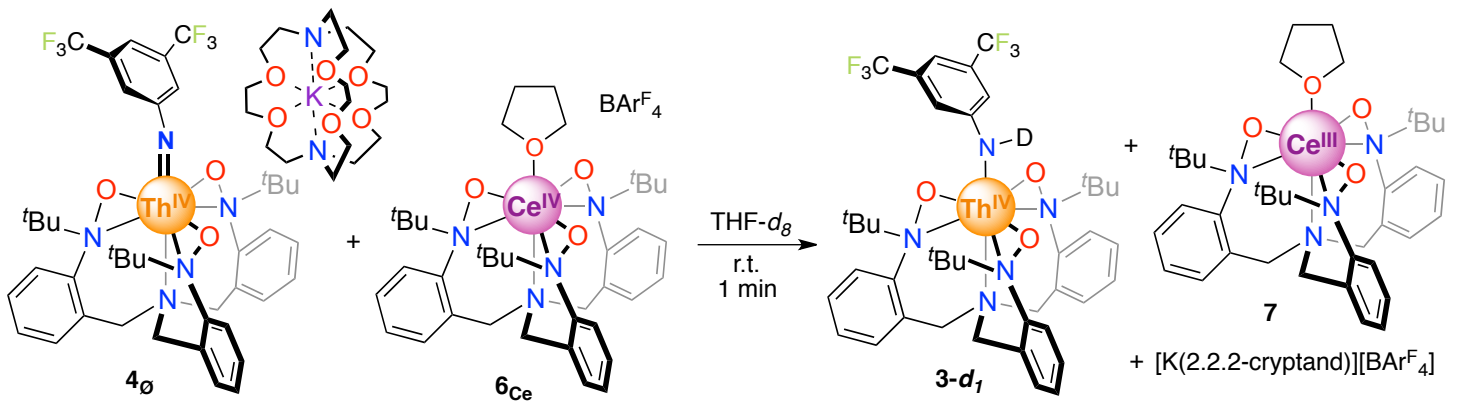

$[\mathrm{K}(2.2 .2-\mathrm{cryptand})][\mathrm{Th}=\mathrm{NAr} F(\operatorname{TriNOx})]\left(\mathbf{4}_{\odot}\right)$

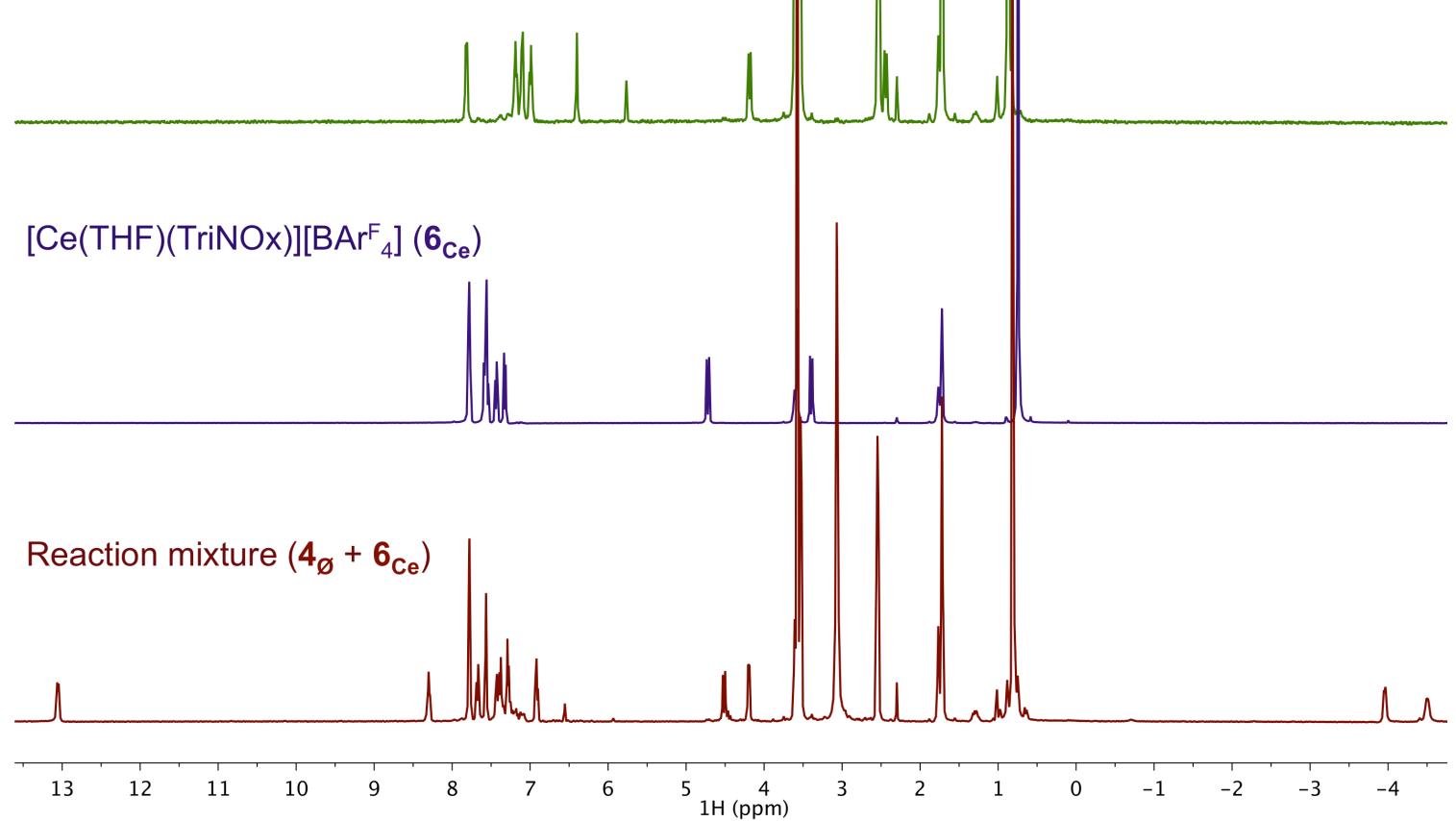

Figure S29. Overlay of the ${ }^{1} \mathrm{H}$ NMR spectra in THF- $d_{8}\left({ }^{*}\right)$ of $\mathbf{4}_{\varnothing}$ (top), $\mathbf{6}_{\mathrm{Ce}}$ (middle), and the reaction mixture (bottom) demonstrating the full conversion of the starting materials. 


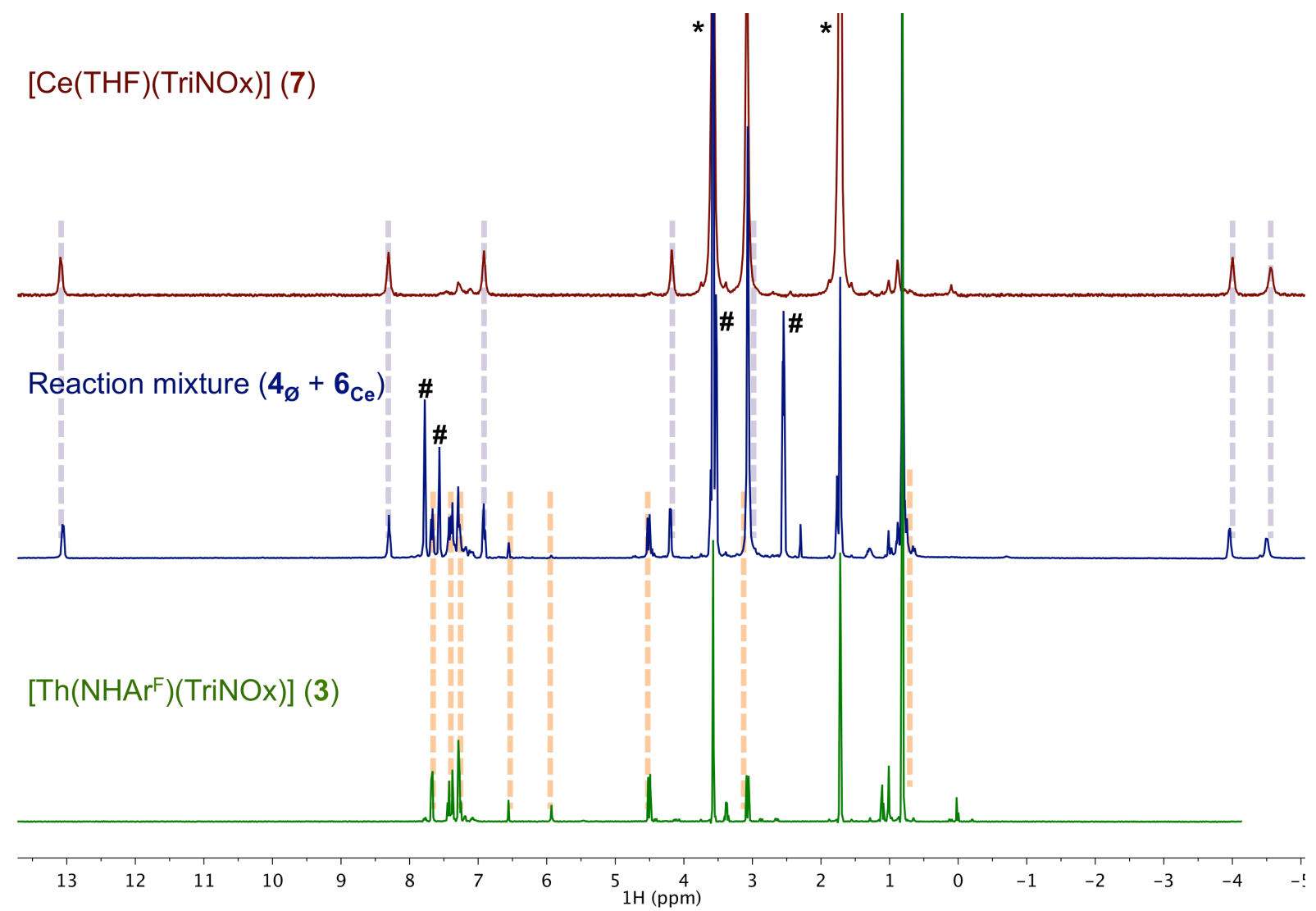

Figure S30. Overlay of the ${ }^{1} \mathrm{H}$ NMR spectra in THF- $d_{8}(*)$ of 7 (top), the reaction mixture (middle), and 3 (bottom). Dashed lines are employed to demonstrate the correspondences. Signals marked with \# are associated with the side product $[\mathrm{K}(2.2 .2$-cryptand $)]\left[\mathrm{BAr}{ }_{4}^{\mathrm{F}}{ }_{4}\right.$.

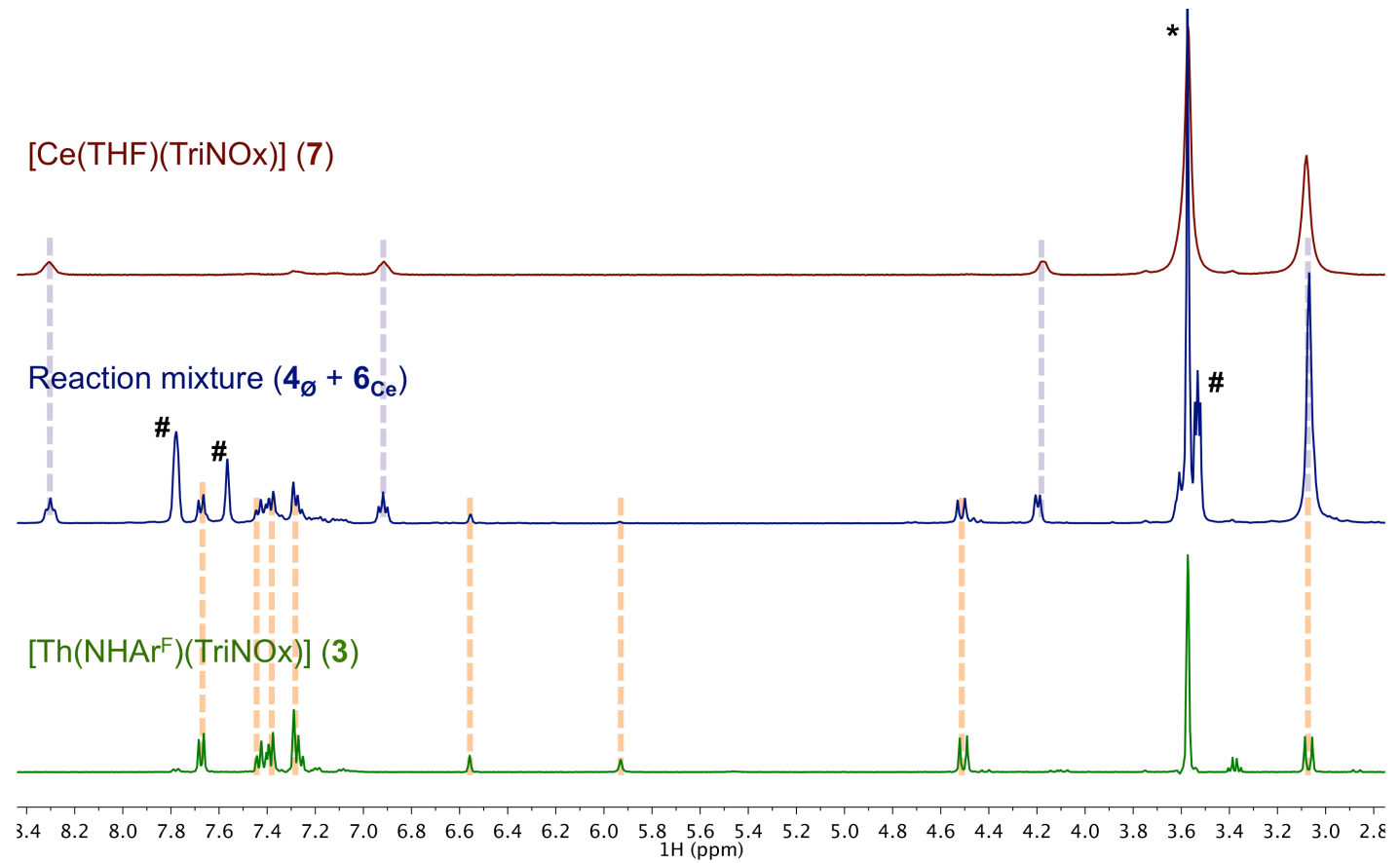

Figure S31. Close-up on the diamagnetic region of Figure S30. 


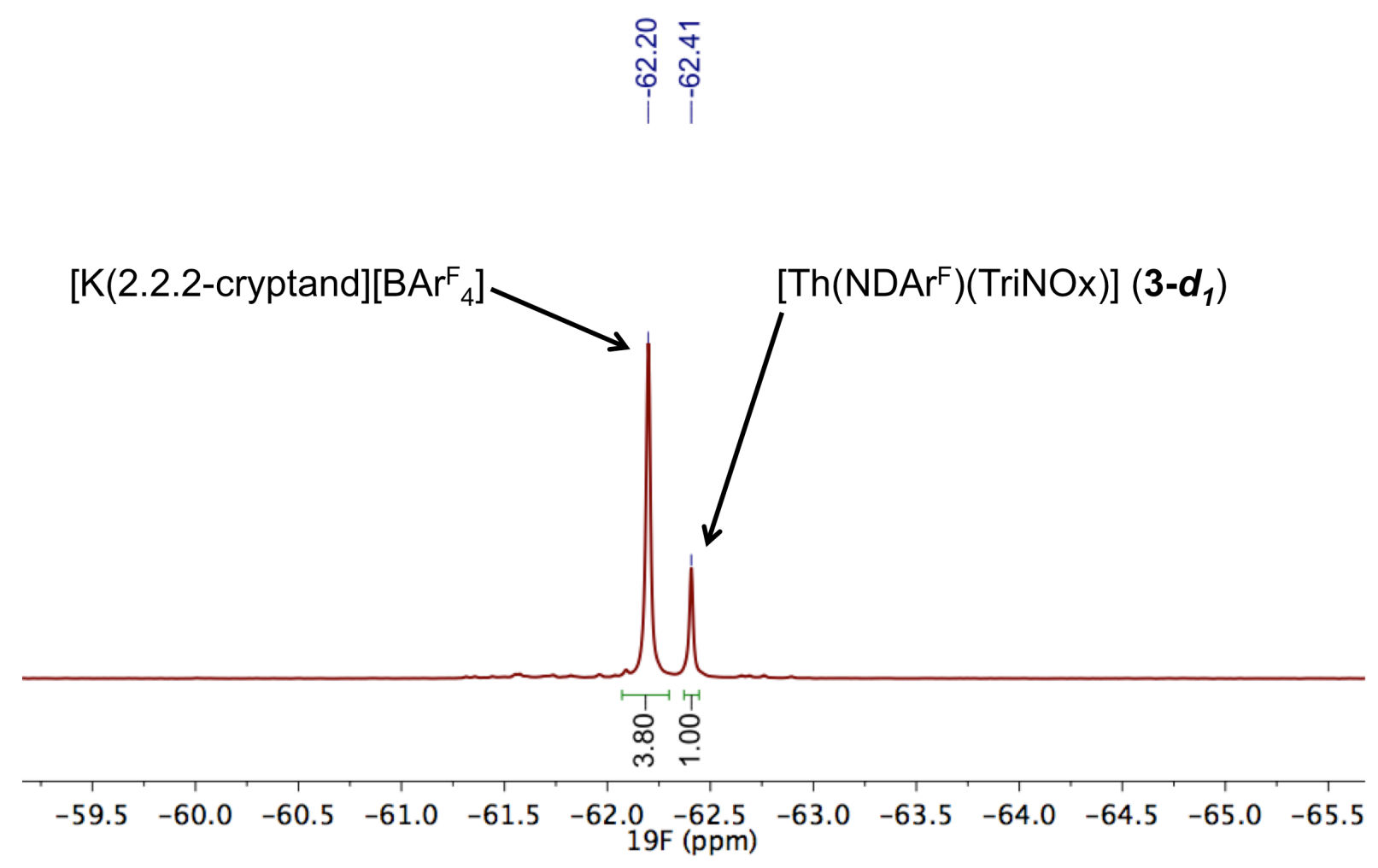

Figure S32. ${ }^{19} \mathrm{~F}\left\{{ }^{1} \mathrm{H}\right\}$ NMR spectrum of the reaction mixture. 
- Reaction of $\mathbf{4}_{\mathrm{Li}}$ and $\mathbf{6}_{\mathrm{Ce}}$ in THF- $d_{8}$ :
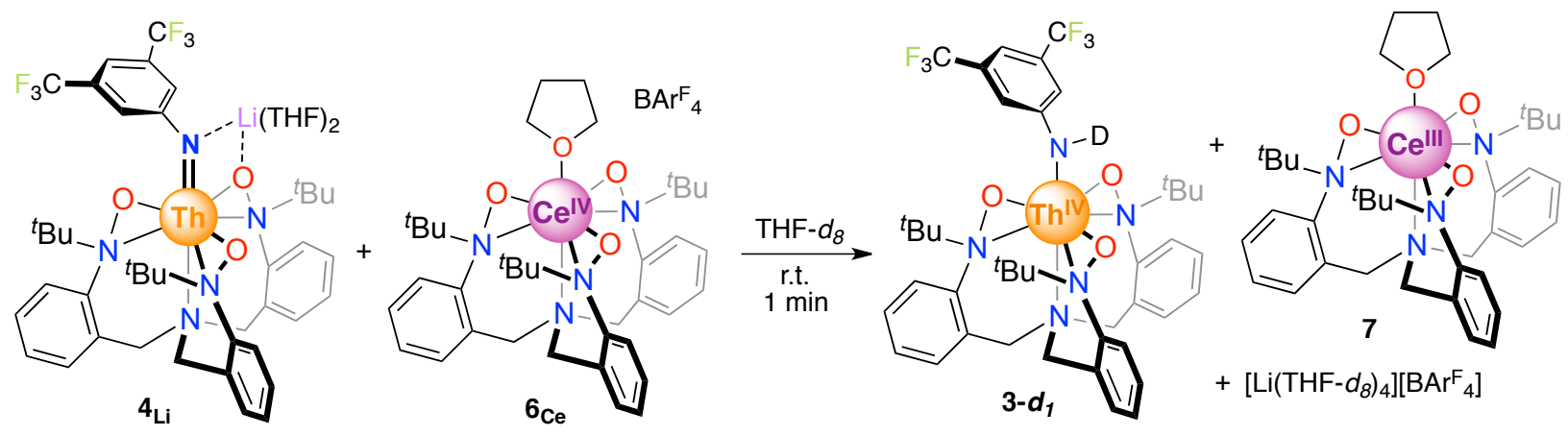

$[\mathrm{Ce}(\mathrm{THF})(\mathrm{TriNOx})](7)$

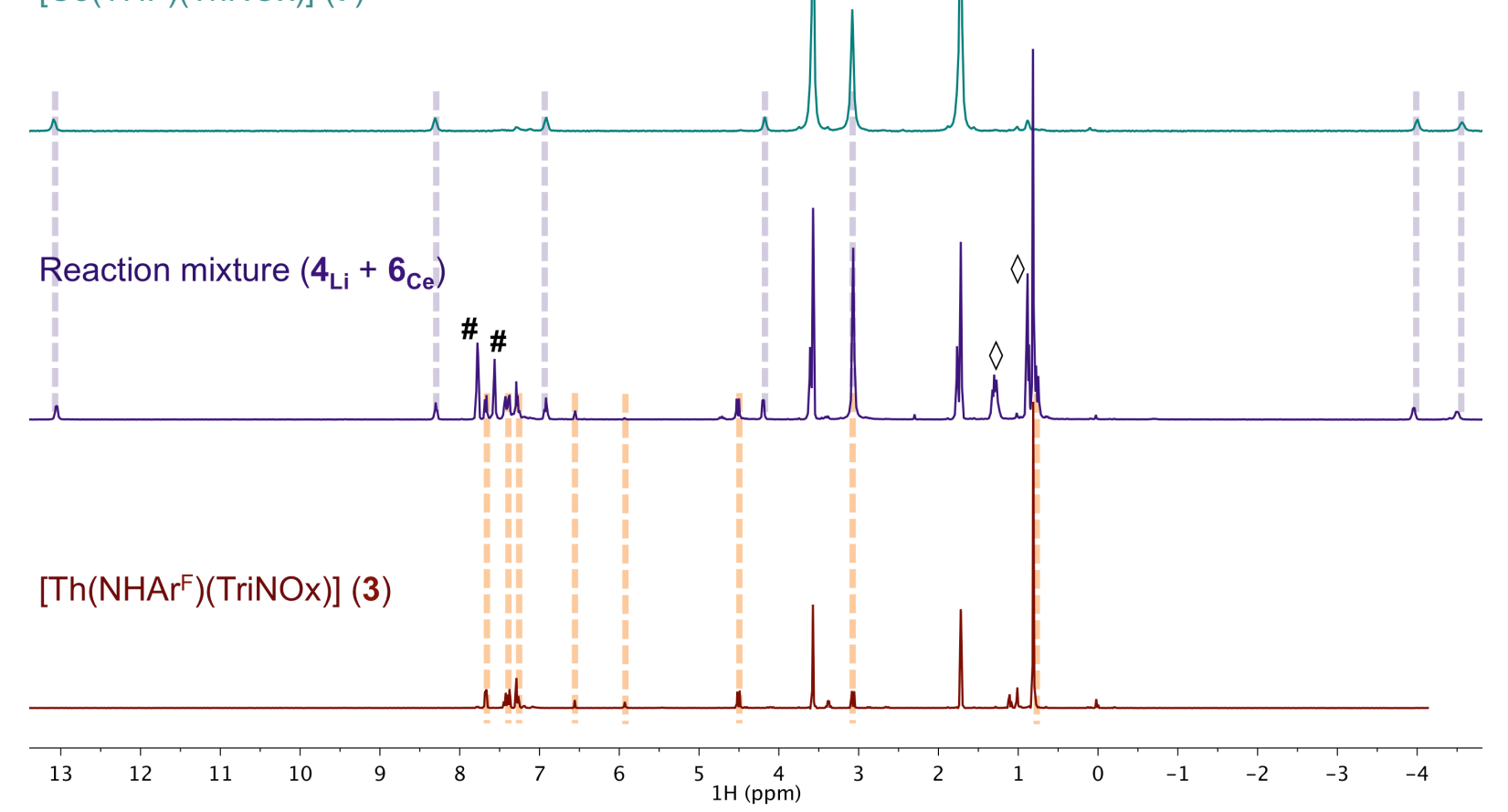

Figure S33. Overlay of the ${ }^{1} \mathrm{H}$ NMR spectra in THF- $d_{8}\left({ }^{*}\right)$ of 7 (top), the reaction mixture (middle), and 3 (bottom). Dashed lines are employed to demonstrate the correspondences. Signals marked with \# are associated with the side product $\left[\mathrm{Li}\left(\mathrm{THF}-d_{8}\right)\right]\left[\mathrm{BAr}_{4}^{\mathrm{F}}\right] . \diamond$ denotes an impurity of $n$-pentane. 
- Reaction of $\mathbf{4}_{\varnothing}$ and $\mathbf{6}_{\mathrm{Ce}}$ in toluene- $d_{8}$ :
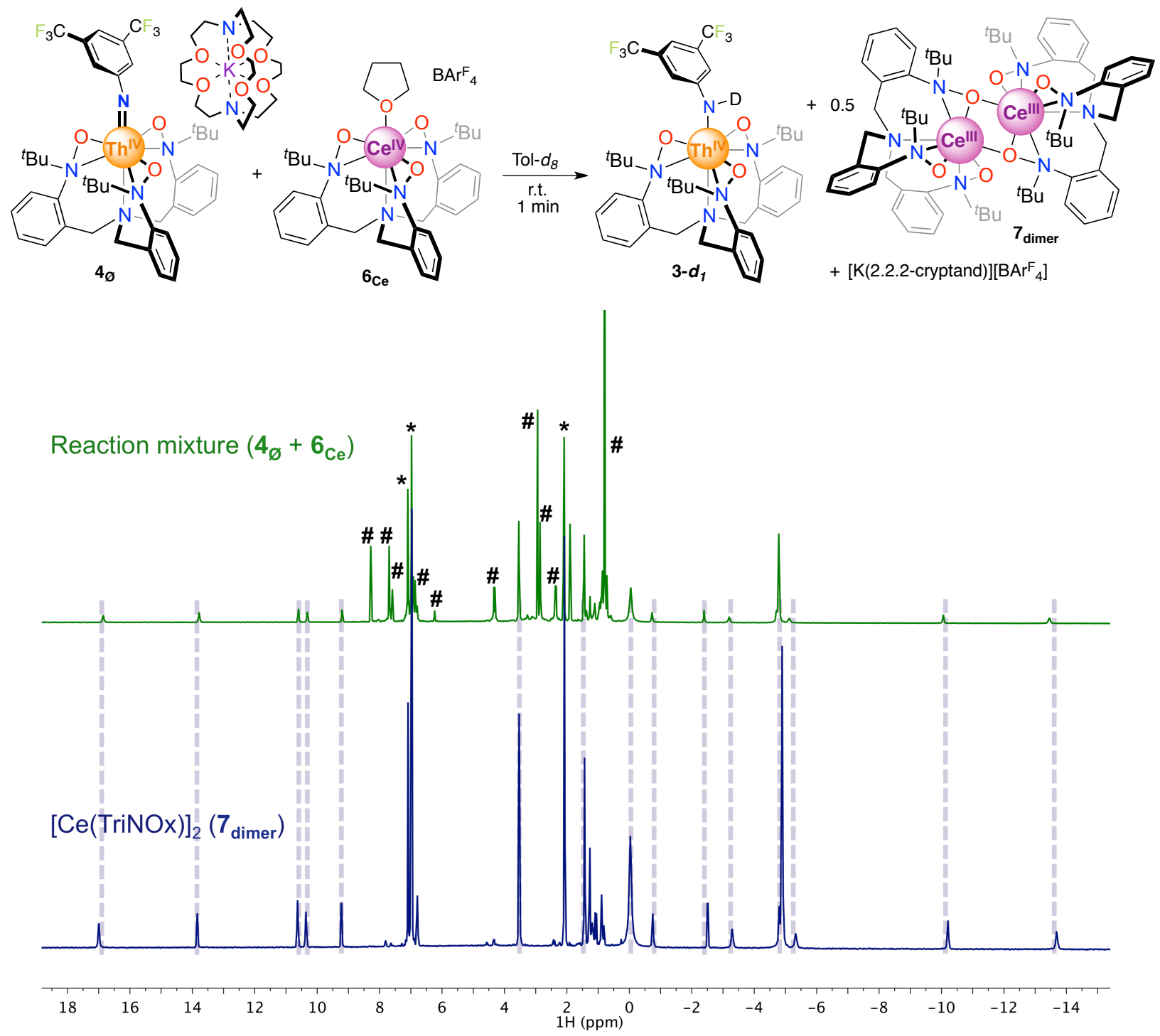

Figure S34. Overlay of the ${ }^{1} \mathrm{H}$ NMR spectra in toluene- $d_{8}\left({ }^{*}\right)$ of $7_{\text {dimer }}$ (bottom) and the reaction mixture (top). Dashed lines are employed to demonstrate the correspondences. Signals marked with \# are associated with $3-\boldsymbol{d}_{\boldsymbol{l}}$ and the side product [K(2.2.2-cryptand)][BAr $\left.{ }_{4}^{\mathrm{F}}\right]$. 


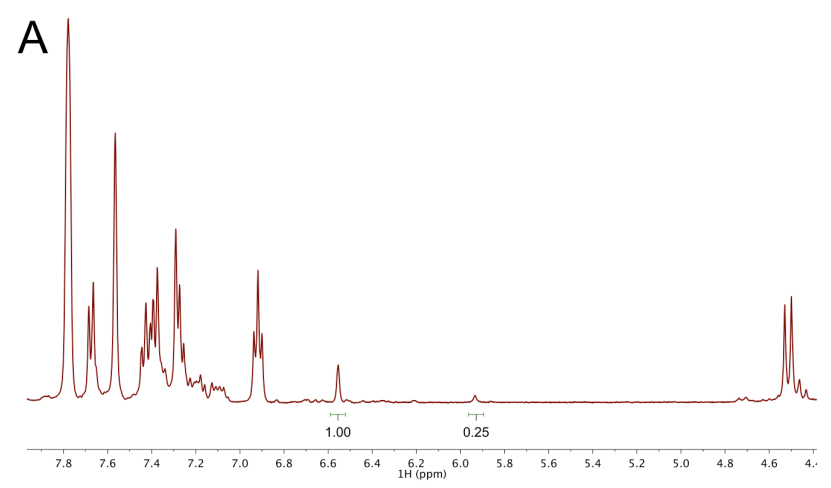

B

C
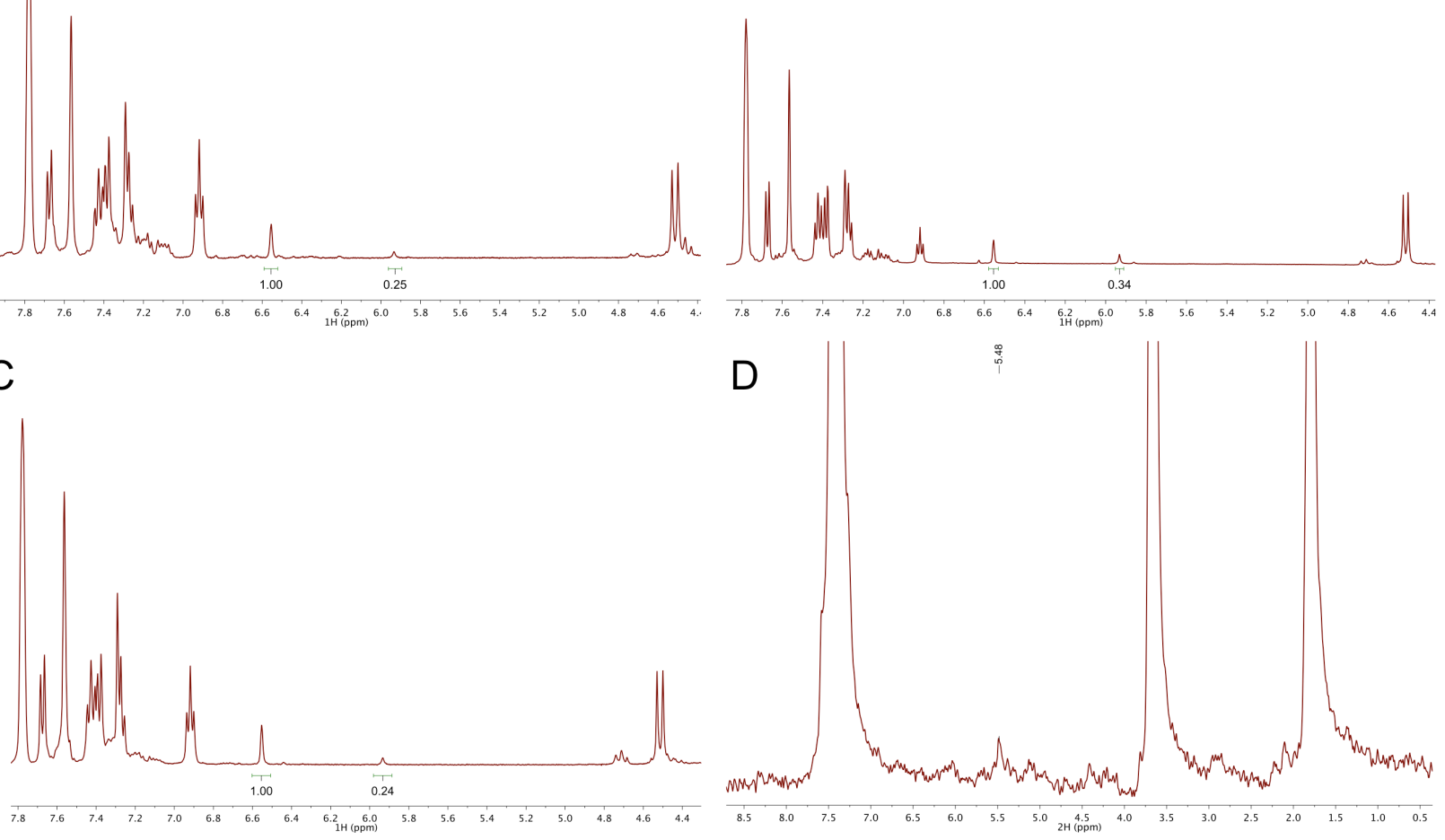

Figure S35. Reduced integration for the NH proton of 3- $\boldsymbol{d}_{1}$ as determined by ${ }^{1} \mathrm{H}$ NMR in THF- $d_{8}$ for the reaction of $\mathbf{4}_{\boldsymbol{g}}+\mathbf{6}_{\mathrm{Ce}}(\mathrm{A} \& \mathrm{C})$ and the reaction $\mathbf{4}_{\mathrm{Li}}+\mathbf{6}_{\mathrm{Ce}}$ (B). ${ }^{2} \mathrm{H}$ NMR spectra of $\mathbf{3}-\boldsymbol{d}_{\boldsymbol{l}}$ redissolved in THF- $h_{8}$ with a drop of $\mathrm{C}_{6} \mathrm{D}_{6}(\mathrm{D})$. 
Computational Details and Supplementary Data. Density Functional Theory (DFT) calculations were carried out using the Gaussian '09 suite (revision D.01). ${ }^{14}$ The hydrid functional combining Becke's 3-parameter exchange functional combined with the Lee-YangParr correlation functional (B3LYP) was employed. ${ }^{15}$ Pople's 6-31-G* basis sets as implemented in Gaussian ` 09 were employed for light atoms $\left(\mathrm{H}, \mathrm{Li}, \mathrm{C}, \mathrm{N}, \mathrm{O}\right.$, and F). ${ }^{17}$ Cerium and thorium atoms were respectively treated with a 28 -electron or 60 -electron small core pseudo-potentials associated with the Stuttgart-Bonn variety of natural orbital incorporating quasi-relativistic effects. ${ }^{18}$ Structures were optimized without constraints; convergence criteria were kept to their default values. All stationary points were verified to possess zero imaginary frequencies by analytical vibrational frequency calculations. Thermochemistry calculations were performed at 298.15 K. Electronic structures calculations (NBO, TD-DFT) were performed from the gasphase optimized structures. During the calculations of the reaction coordinates, dispersion effects and solvation effects were included using respectively the D3 empirical correction proposed by Grimme, ${ }^{16}$ and the conductor-like polarizable continuum model (C-PCM) for THF. ${ }^{19}$ Molecular structures and orbitals were rendered using the Chemcraft software. ${ }^{20}$ The NBO 6.0 package was used to obtain natural charges and to determine the orbital contributions. ${ }^{21}$ The supplemental file (.xyz) contains the computed Cartesian coordinates of all of the molecules reported in this study. The file may be opened as a text file to read the coordinates or opened directly by a molecular modeling program such as Mercury (version 3.3 or later, http://www.ccdc.cam.ac.uk/pages/Home.aspx) for visualization and analysis. 

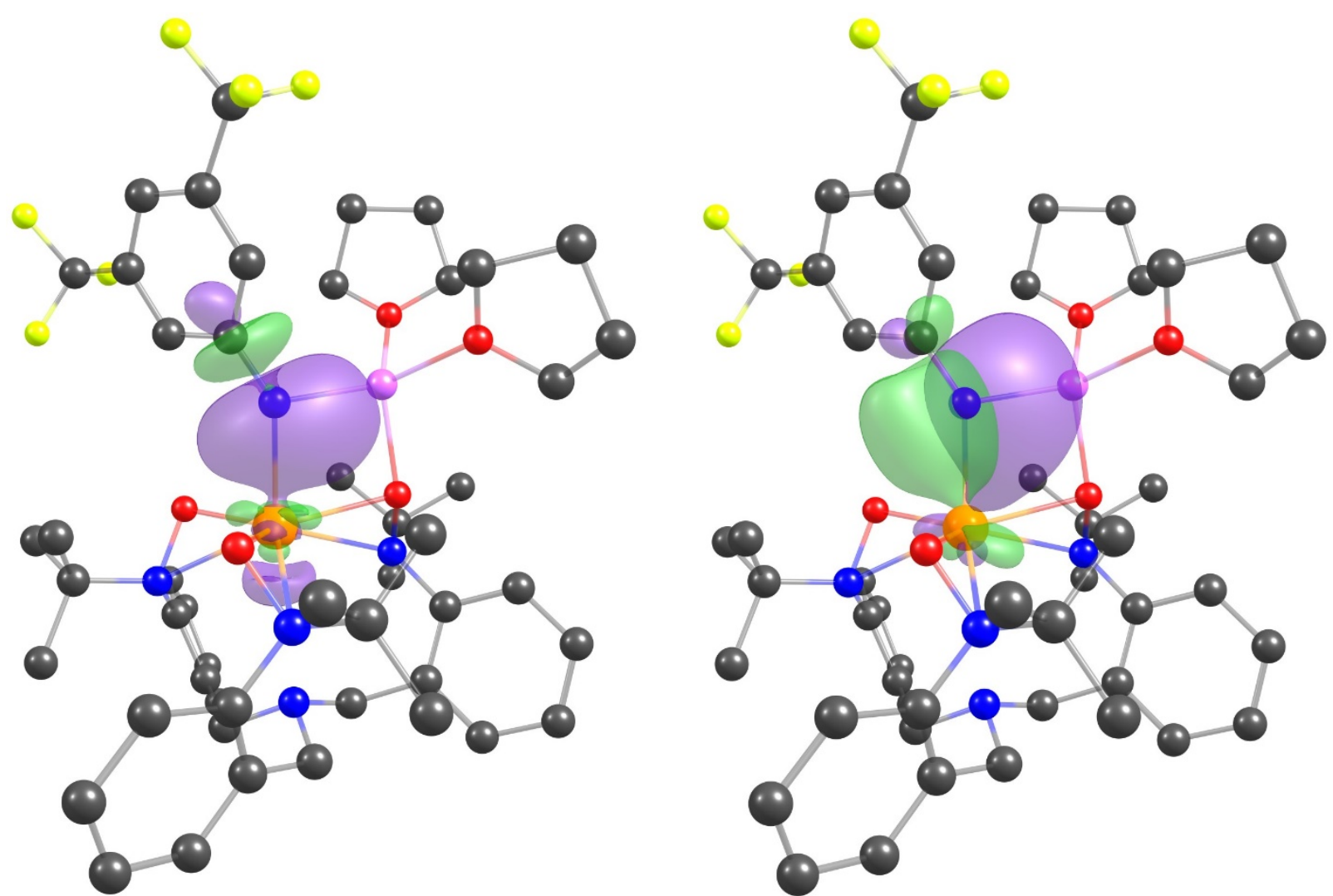

Figure S36. Natural Bonding Orbitals for the $\sigma$ (left) and $\pi$ (right) bonds for $\mathbf{4}_{\mathbf{L i}}$.
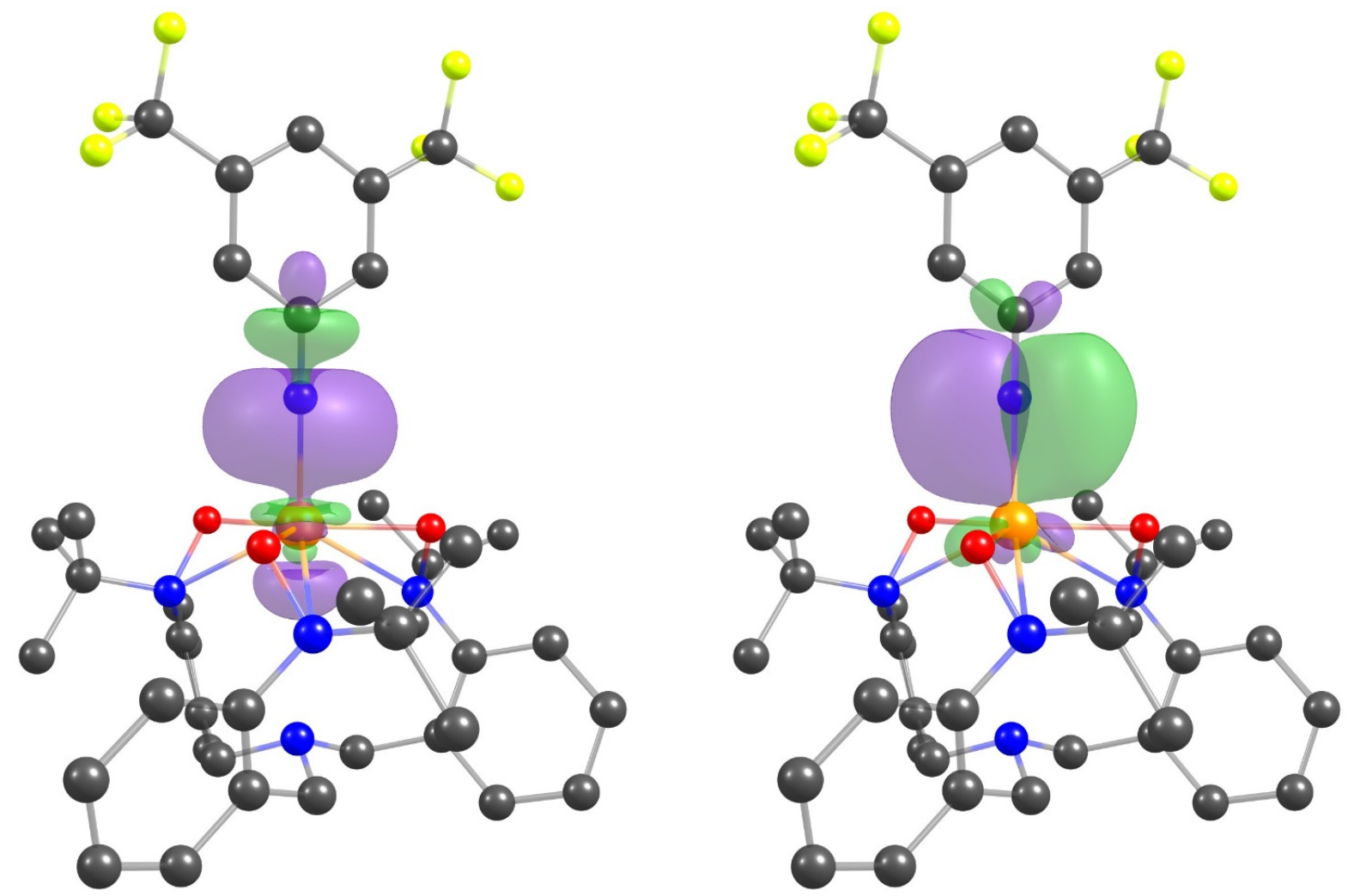

Figure S37. Natural Bonding Orbitals for the $\sigma$ (left) and $\pi$ (right) bonds for $\mathbf{4}_{\varnothing}{ }^{-}$. 


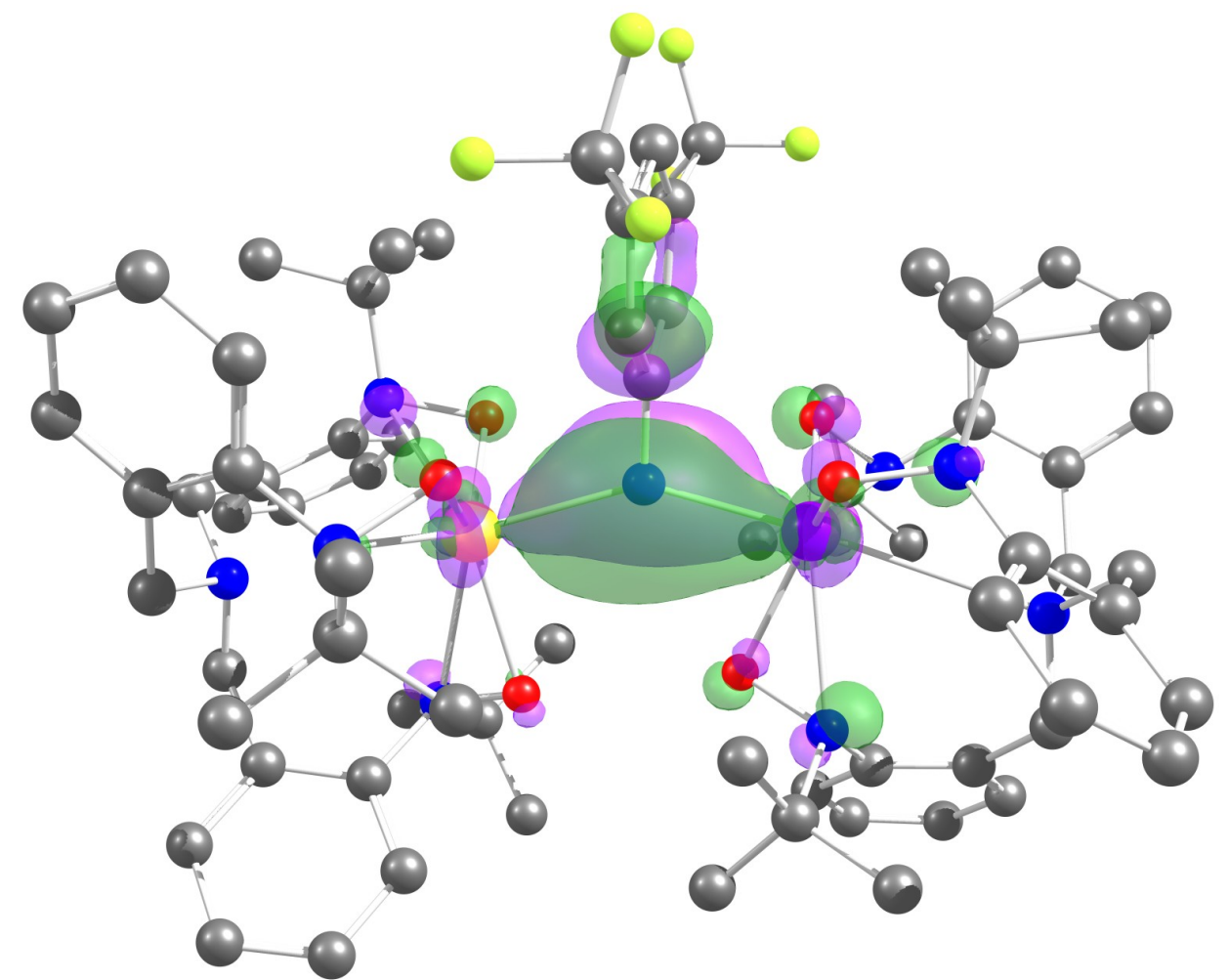

Figure S38. HOMO-1 (Kohn-Sham) of ${ }^{\mathbf{1}} \mathbf{8}$ demonstrating a bridging imido $\pi$-bond.

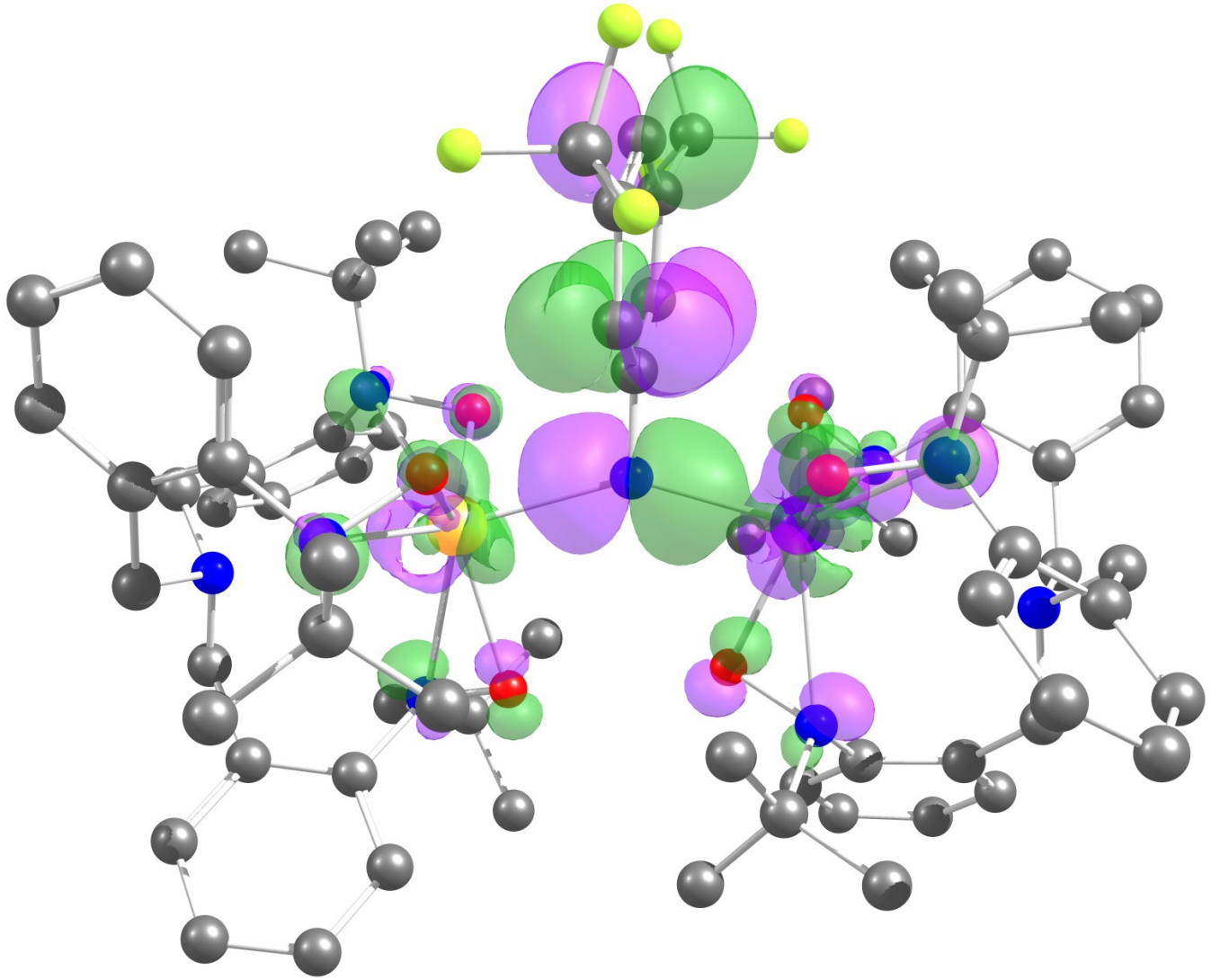

Figure S39. HOMO (Kohn-Sham) of ${ }^{\mathbf{1}} \mathbf{8}$ demonstrating a bridging imido $\sigma$-bond. 


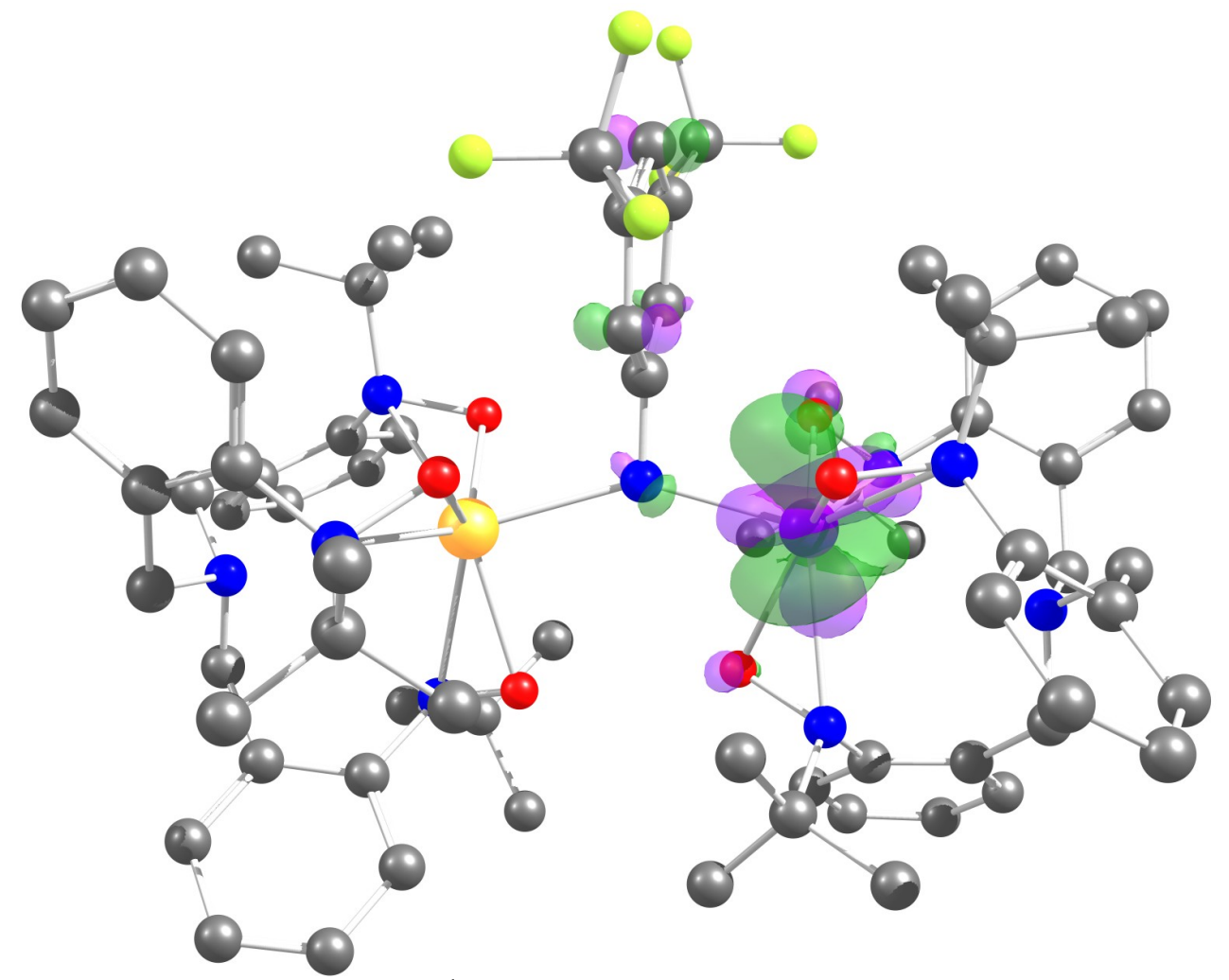

Figure S40. LUMO (Kohn-Sham) of ${ }^{1} \mathbf{8}$ suggesting a cerium-based reduction locus.

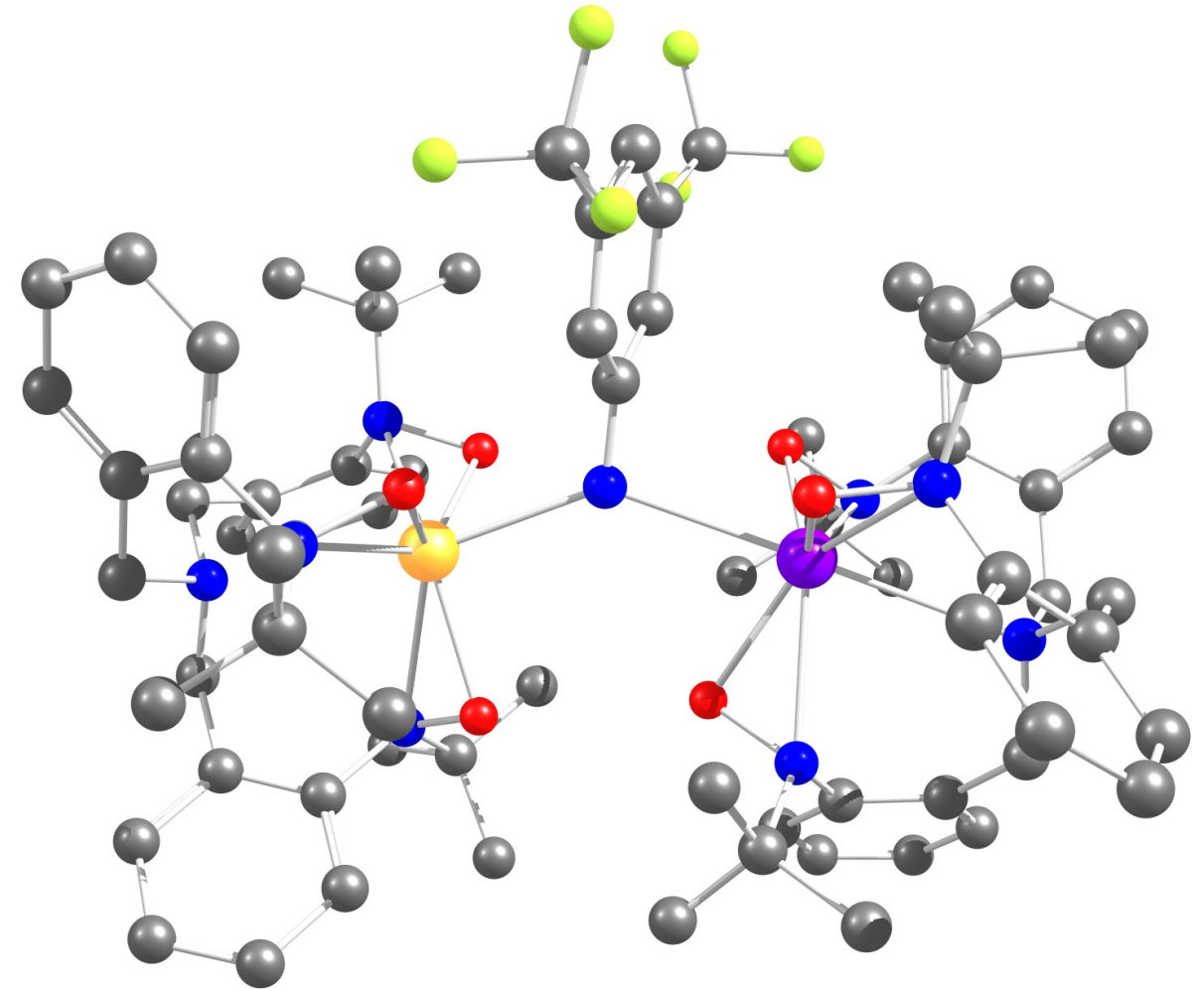

Figure S41. Structure of ${ }^{3} \mathbf{8}$ pre-optimized with a reduced convergence criterion (conver $=6$ ), full optimization and frequency calculations were unsuccessful due to computational limitations. 


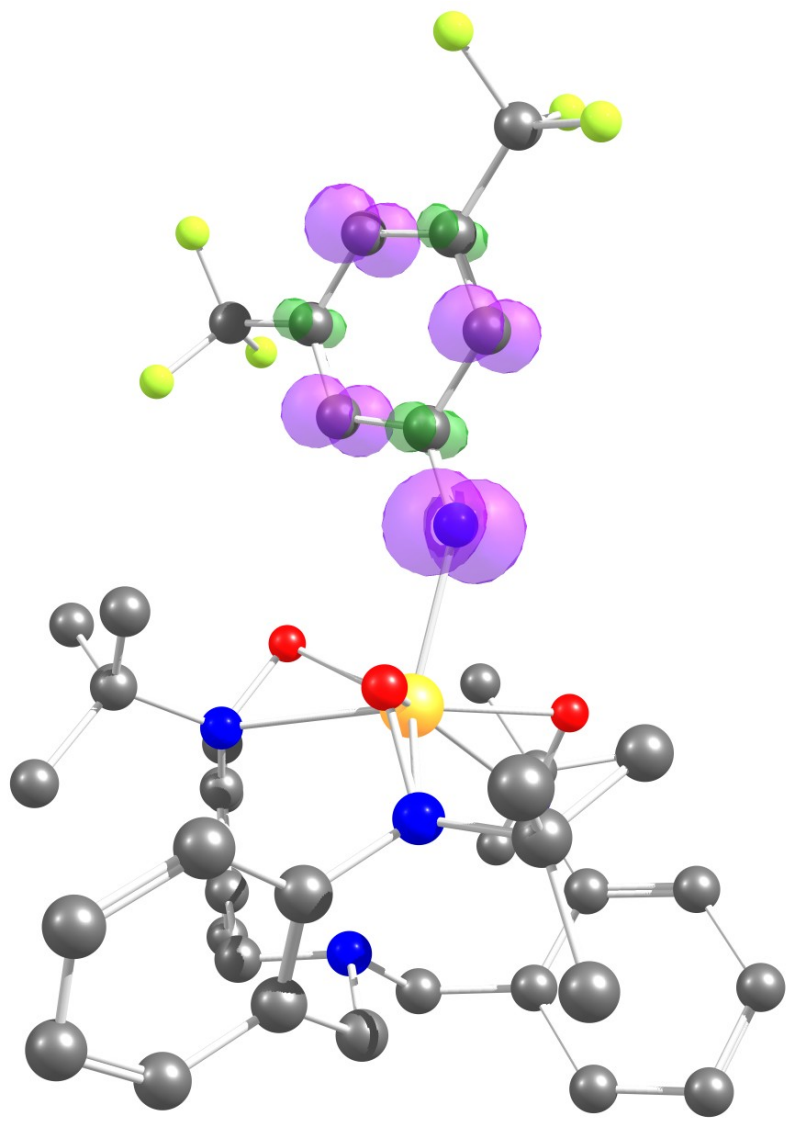

Figure S42. Spin density plot of ${ }^{2} \mathbf{9}$ demonstrating the iminyl character.

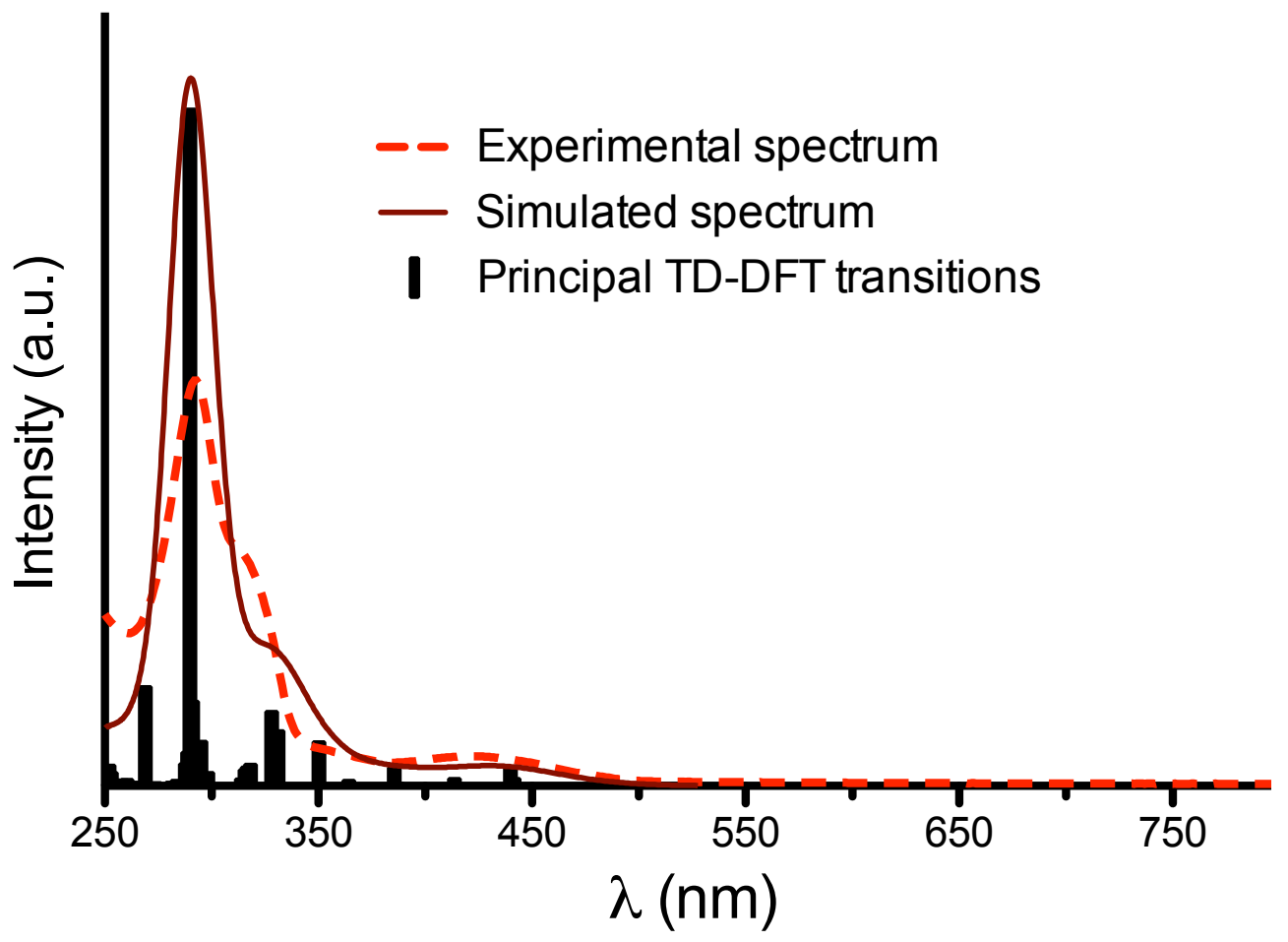

Figure S43. Experimental UV-visible spectrum of $\mathbf{4}_{\varnothing}$ and TD-DFT predicted spectrum of $\mathbf{4}_{\varnothing}{ }^{-}$. 

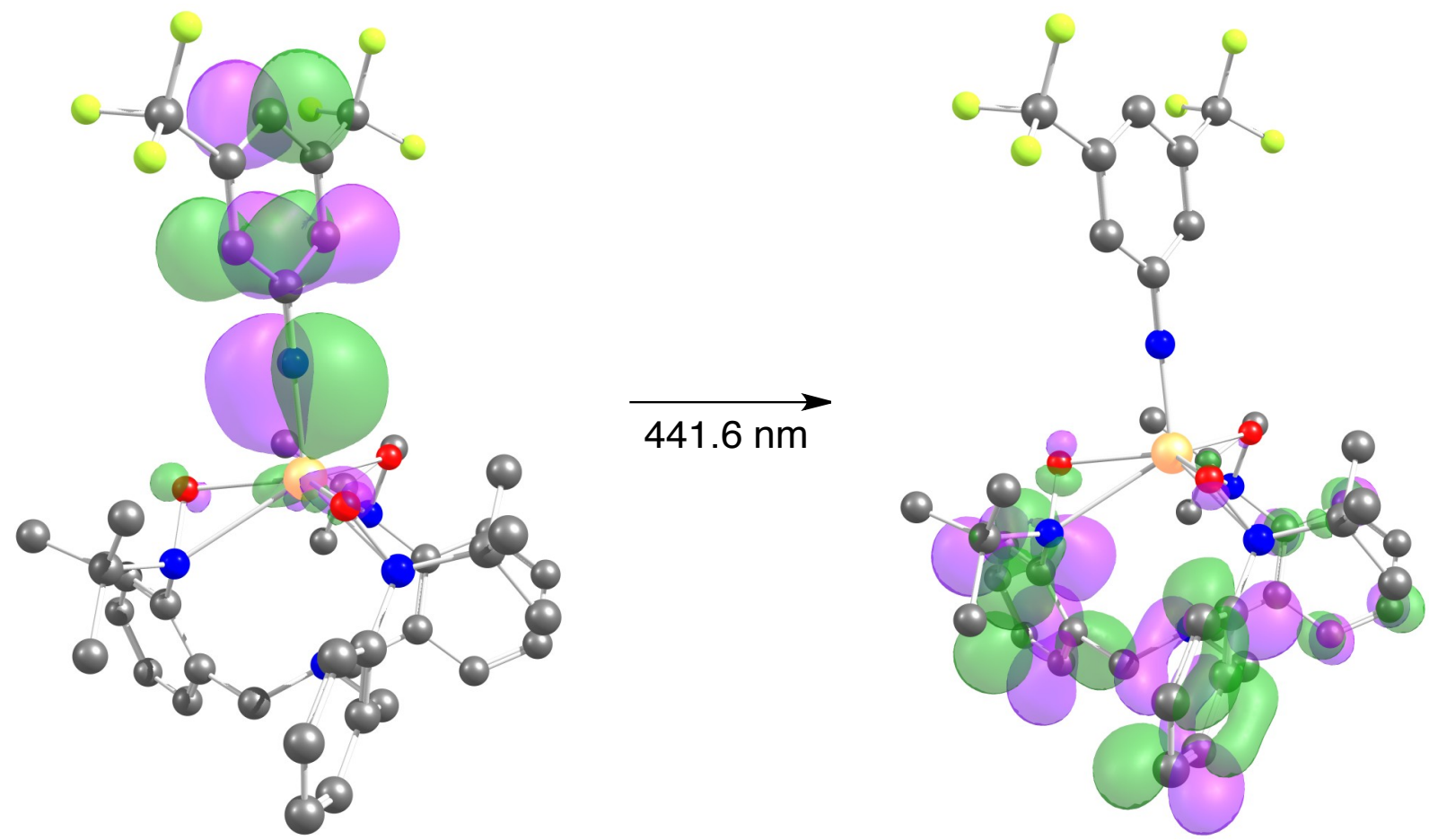

Figure S44. Calculated natural transition orbitals for the transition at $441.6 \mathrm{~nm}$ from the HOMO $\left(\pi_{\mathrm{Th}=\mathrm{N}}\right)$ to the $\mathrm{LUMO}+1\left(\pi^{*}{ }_{\text {TriNOx }}\right)$.
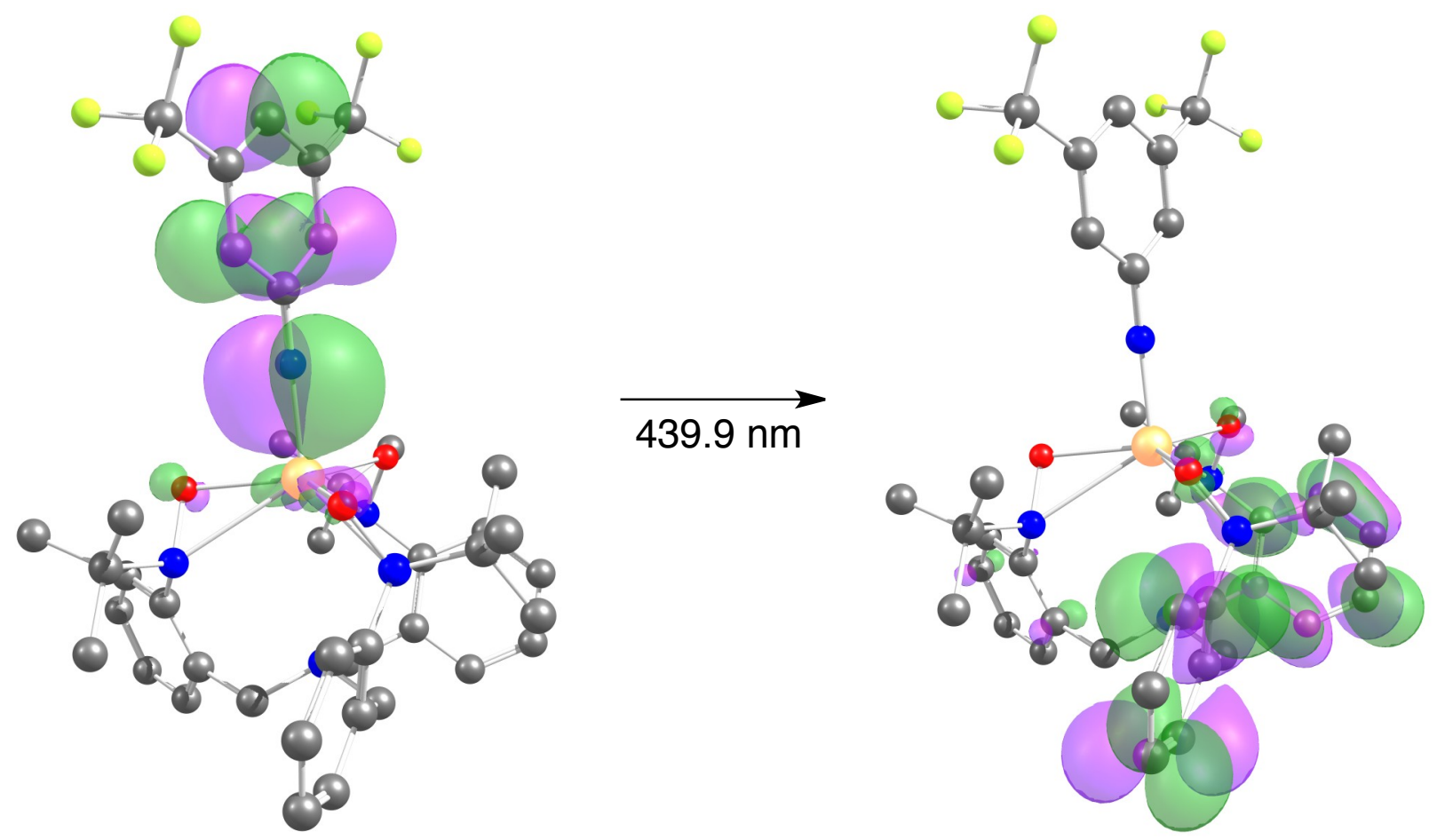

Figure S45. Calculated natural transition orbitals for the transition at $439.9 \mathrm{~nm}$ from the HOMO $\left(\pi_{\mathrm{Th}=\mathrm{N}}\right)$ to the $\mathrm{LUMO}+2\left(\pi^{*}{ }_{\mathrm{TriNO}}\right)$. 


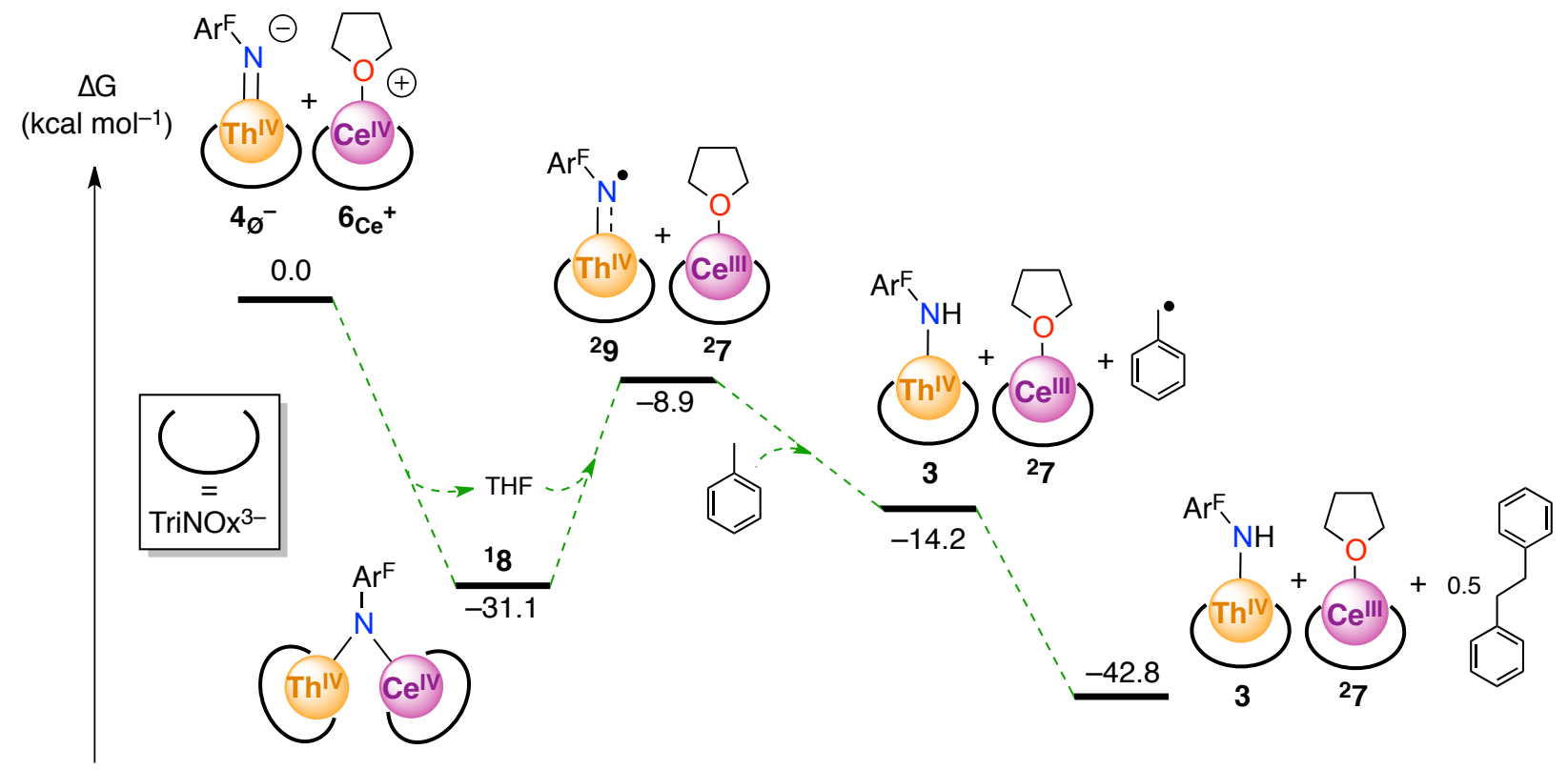

Figure S46. Reaction coordinates accounting for the formation of an intermediate benzyl radical. 
Table S5. Thermochemistry data for the computed structures (in Hartrees at 298.15 K).

\begin{tabular}{c|cccc} 
Compound & $\mathbf{E}^{\mathrm{a}}$ & $\mathbf{H}^{\mathrm{b}}$ & $\mathbf{G}^{\mathrm{c}}$ & $\mathbf{C - P C M}^{\mathrm{d}}$ \\
\hline \hline $\mathbf{4}_{\mathbf{L i}}$ (NBO) & -3568.926087 & -3568.925143 & -3569.106156 & $/$ \\
$\mathbf{4}_{\boldsymbol{}}{ }^{-}$(NBO) & -3096.700294 & -3096.699350 & -3096.847526 & $/$ \\
$\mathbf{4}_{\boldsymbol{}}{ }^{-}$ & -3096.873982 & -3096.873038 & -3097.017428 & -3097.835844 \\
$\mathbf{6}_{\mathbf{C}{ }^{+}}$ & -2436.025603 & -2436.024659 & -2436.145807 & -2436.975771 \\
$\mathbf{5}_{\boldsymbol{}}{ }^{-}$ & -3164.232295 & -3164.231351 & -3164.374172 & -3165.184229 \\
$\mathbf{6}_{\mathbf{T h}^{+}}$ & -2368.67041 & -2368.669465 & -2368.791589 & -2369.628733 \\
${ }^{\mathbf{8}}$ & -5300.713803 & -5300.712859 & -5300.934145 & -5302.41531 \\
${ }^{2} \mathbf{9}$ & -3096.797342 & -3096.796398 & -3096.941222 & -3097.714499 \\
${ }^{2} \mathbf{7}$ & -2436.195119 & -2436.194175 & -2436.317706 & -2437.108435 \\
$\mathbf{3}$ & -3097.445936 & -3097.444992 & -3097.590132 & -3098.37774 \\
THF & -232.330497 & -232.329553 & -232.363914 & -232.4557831 \\
Toluene & -271.434338 & -271.433394 & -271.470918 & -271.5711999 \\
Benzyl radical & -270.796034 & -270.79509 & -270.831348 & -270.9192071 \\
Bibenzyl & -541.689438 & -541.688493 & -541.741457 & -541.9446825
\end{tabular}

a) Sum of electronic and thermal Energies; b) Sum of electronic and thermal Enthalpies; c) Sum of electronic and thermal Free Energies; d) SCF Energies from C-PCM single-point.

Table S6. Lowest frequencies $\left(\mathrm{cm}^{-1}\right)$ of the computed structures.

\begin{tabular}{c|ccc} 
Compound & Freq 1 & Freq 2 & Freq 3 \\
\hline \hline $\mathbf{4}_{\mathbf{L i}}$ (NBO) & 9.2055 & 13.2860 & 15.2578 \\
$\mathbf{4}_{\varnothing}{ }^{-}$(NBO) & 5.0458 & 10.9598 & 13.4510 \\
$\mathbf{4}^{-}$ & 7.4168 & 12.0570 & 19.1546 \\
$\mathbf{6}_{\mathbf{C e}}^{+}$ & 16.9564 & 36.8137 & 39.9230 \\
$\mathbf{5}^{-}$ & 6.0356 & 12.9549 & 16.7845 \\
$\mathbf{6}_{\mathbf{T h}}^{+}$ & 26.2330 & 35.9703 & 37.8092 \\
${ }^{\mathbf{1}} \mathbf{8}$ & 14.2304 & 17.0826 & 19.5125 \\
${ }^{2} \mathbf{9}$ & 8.7981 & 10.1008 & 15.4788 \\
${ }^{2} \mathbf{7}$ & 18.2627 & 34.3403 & 38.1948 \\
$\mathbf{3}$ & 2.6138 & 8.3086 & 15.5452 \\
THF & 45.7545 & 264.1323 & 585.8127 \\
Toluene & 48.6323 & 210.5887 & 353.4773 \\
Benzyl radical & 202.4084 & 370.0182 & 392.6219 \\
Bibenzyl & 24.4632 & 36.8323 & 51.5013
\end{tabular}




\section{References.}

(1) Bogart, J. A.; Lippincott, C. A.; Carroll, P. J.; Schelter, E. J. An Operationally Simple Method for Separating the Rare-Earth Elements Neodymium and Dysprosium, Angew. Chem. Int. Ed. 2015, 54, 8222-8225.

(2) Cantat, T.; Scott, B. L.; Kiplinger, J. L. Convenient access to the anhydrous thorium tetrachloride complexes $\mathrm{ThCl}_{4}(\mathrm{DME})_{2}, \quad \mathrm{ThCl}_{4}(1,4-\text { dioxane })_{2}$ and $\mathrm{ThCl}_{4}(\mathrm{THF})_{3.5}$ using commercially available and inexpensive starting materials, Chem. Commun. 2010, 46, 919.

(3) Bailey, P. J.; Coxall, R. A.; Dick, C. M.; Fabre, S.; Henderson, L. C.; Herber, C.; Liddle, S. T.; Loroño-González, D.; Parkin, A.; Parsons, S. The First Structural Characterisation of a Group 2 Metal Alkylperoxide Complex: Comments on the Cleavage of Dioxygen by Magnesium Alkyl Complexes, Chemistry - A European Journal 2003, 9, 4820-4828.

(4) Martínez-Martínez, A. J.; Weller, A. S. Solvent-free anhydrous $\mathrm{Li}^{+}, \mathrm{Na}^{+}$and $\mathrm{K}^{+}$salts of $\left[\mathrm{B}\left(3,5-\left(\mathrm{CF}_{3}\right)_{2} \mathrm{C}_{6} \mathrm{H}_{3}\right)_{4}\right]^{-},\left[\mathrm{BArF}_{4}\right]^{-}$. Improved synthesis and solid-state structures, Dalton Trans. 2019, 48, 3551-3554.

(5) Thomson, R. K.; Scott, B. L.; Morris, D. E.; Kiplinger, J. L. Synthesis, structure, spectroscopy and redox energetics of a series of uranium(IV) mixed-ligand metallocene complexes, C. R. Chimie 2010, 13, 790-802.

(6) Bogart, J. A.; Lippincott, C. A.; Carroll, P. J.; Booth, C. H.; Schelter, E. J. Controlled Redox Chemistry at Cerium within a Tripodal Nitroxide Ligand Framework, Chem. Eur. J. 2015, 21, 17850-17859.

(7) Bruker $A P E X 2$, v2014.11-0; Bruker AXS Inc.: Madison, Wisconsin, 2012.

(8) Bruker $A P E X 3$, v2016.1-0; Bruker AXS Inc.: Madison, Wisconsin, 2015.

(9) Bruker SAINT, v8.37a; Bruker AXS Inc.: Madison, Wisconsin, 2012.

(10) Sheldrick, G. M. SHELXT - Integrated space-group and crystal-structure determination, Acta Crystallogr. Sect. A: Found. Crystallogr. 2015, 71, 3-8.

(11) Bruker $S A D A B S$, v2014/5; Bruker AXS Inc.: Madison, Wisconsin, 2001.

(12) Sheldrick, G. M. Crystal structure refinement with SHELXL, Acta Crystallogr. Sect. C: Cryst. Struct. Commun. 2015, 71, 3-8.

(13) (a) Fang, H.; Cole, B. E.; Qiao, Y.; Bogart, J. A.; Cheisson, T.; Manor, B. C.; Carroll, P. J.; Schelter, E. J. Electro-kinetic Separation of Rare Earth Elements Using a Redox-Active Ligand, Angew. Chem. Int. Ed. 2017, 56, 13450-13454. (b) McSkimming, A.; Su, J.; Cheisson, T.; Gau, M. R.; Carroll, P. J.; Batista, E. R.; Yang, P.; Schelter, E. J. Coordination Chemistry of a Strongly-Donating Hydroxylamine with Early Actinides: An Investigation of Redox Properties and Electronic Structure, Inorg. Chem. 2018, 57, 4387-4394.

(14) Gaussian 09, Revision D.01, Frisch, M. J.; Trucks, G. W.; Schlegel, H. B.; Scuseria, G. E.; Robb, M. A.; Cheeseman, J. R.; Scalmani, G.; Barone, V.; Mennucci, B.; Petersson, G. A.; Nakatsuji, H.; Caricato, M.; Li, X.; Hratchian, H. P.; Izmaylov, A. F.; Bloino, J.; Zheng, G.; Sonnenberg, J. L.; Hada, M.; Ehara, M.; Toyota, K.; Fukuda, R.; Hasegawa, J.; Ishida, M.; Nakajima, T.; Honda, Y.; Kitao, O.; Nakai, H.; Vreven, T.; Montgomery Jr., J. A.; Peralta, J. E.; Ogliaro, F.; Bearpark, M.; Heyd, J. J.; Brothers, E.; Kudin, K. N.; Staroverov, V. N.; Kobayashi, R.; Normand, J.; Raghavachari, K.; Rendell, A.; Burant, J. C.; Iyengar, S. S.; Tomasi, J.; Cossi, M.; Rega, N.; Millam, N. J.; Klene, M.; Knox, J. E.; Cross, J. B.; Bakken, V.; Adamo, C.; Jaramillo, J.; Gomperts, R.; Stratmann, R. E.; Yazyev, O.; Austin, A. J.; Cammi, R.; Pomelli, C.; Ochterski, J. W.; Martin, R. L.; Morokuma, K.; Zakrzewski, V. G.; Voth, V.; Cioslowski, J.; Fox, D. J., Gaussian, Inc., Wallingford, CT, 2009. 
(15) (a) Becke, A. D. Density-functional thermochemistry. III. The role of exact exchange, $J$. Chem. Phys. 1993, 98, 5648-5652. (b) Lee, C.; Yang, W.; Parr, R. G. Development of the ColleSalvetti correlation-energy formula into a functional of the electron density, Phys. Rev. B 1988, 37, 785-789. (c) Vosko, S. H.; Wilk, L.; Nusair, M. Accurate spin-dependent electron liquid correlation energies for local spin density calculations: a critical analysis, Can. J. Phys. 1980, 58, 1200-1211. (d) Stephens, P. J.; Devlin, F. J.; Chabalowski, C. F.; Frisch, M. J. Ab Initio Calculation of Vibrational Absorption and Circular Dichroism Spectra Using Density Functional Force Fields, J. Phys. Chem. 1994, 98, 11623-11627.

(16) Grimme, S.; Antony, J.; Ehrlich, S.; Krieg, H. A consistent and accurate ab initio parametrization of density functional dispersion correction (DFT-D) for the 94 elements $\mathrm{H}-\mathrm{Pu}, J$. Chem. Phys. 2010, 132, 154104.

(17) Hehre, W. J.; Ditchfield, R.; Pople, J. A. Self - Consistent Molecular Orbital Methods. XII. Further Extensions of Gaussian-Type Basis Sets for Use in Molecular Orbital Studies of Organic Molecules, J. Chem. Phys. 1972, 56, 2257-2261.

(18) (a) Küchle, W.; Dolg, M.; Stoll, H.; Preuss, H. Energy-adjusted pseudopotentials for the actinides. Parameter sets and test calculations for thorium and thorium monoxide, J. Chem. Phys. 1994, 100, 7535-7542. (b) Cao, X.; Dolg, M. Valence basis sets for relativistic energy-consistent small-core lanthanide pseudopotentials, J. Chem. Phys. 2001, 115, 7348-7355. (c) Cao, X.; Dolg, M. Segmented contraction scheme for small-core lanthanide pseudopotential basis sets, $J$. Mol. Struct.: THEOCHEM 2002, 581, 139-147. (d) Cao, X.; Dolg, M.; Stoll, H. Valence basis sets for relativistic energy-consistent small-core actinide pseudopotentials, J. Chem. Phys. 2003, $118,487-496$.

(19) Cossi, M.; Rega, N.; Scalmani, G.; Barone, V. Energies, structures, and electronic properties of molecules in solution with the C-PCM solvation model, J. Comput. Chem. 2003, 24, 669-681.

(20) Andrienko, G. A. Chemcraft, v1.6. http://www.chemcraftprog.com

(21) Glendening, E. D.; Badenhoop, J. K.; Reed, A. E.; Carpenter, J. E.; Bohmann, J. A.; Morales, C. M.; Weinhold, F. NBO 6.0, University of Wisconsin: Madison, WI, 2001. 